\title{
BECCARIA TRA DIRITTO PENALE ED ECONOMIA PUBBLICA
}

\author{
GIAN PAOLO MASSETTO (*)
}

RIASsunTO. - Scriveva Mario Romani nel 1966: “[...] la sua [di Beccaria] più matura e solida parte [di produzione], ossia il testo delle lezioni milanesi di pubblica economia tenute dal 1769 al 1772 per le sue vedute d'insieme, da trattatista, e le numerose relazioni o «consulte» frutto delle ricerche compiute in quanto membro di organi di governo in cui risaltano le sue doti per le analisi empiriche, resta a lungo, troppo a lungo ignorato in tutto o in parte, o affidata a editori malsicuri, pronti a dare alle stampe manoscritti giudicati non pubblicabili dallo stesso Autore". Il disagio che queste parole denunciano non ha ora più ragion d'essere. E' imminente la pubblicazione dell'edizione critica degli Elementi, mentre è giunta a conclusione la pubblicazione degli Atti di Governo che illustrano al completo l'attività svolta dal Milanese nelle funzioni di consigliere del Supremo Consiglio d'Economia, del Nuovo Magistrato Camerale, di capo del III dipartimento, poi del II, del Consiglio di Governo. Lo studioso è così posto in grado di conoscere e di valutare appieno l'opera di Beccaria teorico e di Beccaria in azione sia in ordine al settore penale che a quello economico. L'oggetto della relazione consiste nel tentativo di determinare se vi sia coerenza tra quanto scritto a tavolino dal Milanese e quanto operato, per così dire, sul campo intorno a temi in cui stretto è il nesso tra diritto penale ed economia pubblica. L'attenzione si soffermerà essenzialmente su argomenti inerenti alla libertà di commercio, dei grani in particolare, alla libera panizzazione, alla disciplina della caccia, dei boschi e delle miniere, all'Ergastolo di Pizzighettone, alla Casa di Correzione di Milano.

$$
* * *
$$

(*) Università degli Studi, Milano.

Viene qui pubblicata la relazione presentata in occasione dell'Incontro di studio, integrata con le parti allora omesse per ragioni tempo e con le note, che si limitano alla citazione delle sole fonti (quelle di letteratura storiografica, che si rinvengono nel corpo del testo, sono determinate dal fatto che esse assumono il rilievo di fonte, diretta o indiretta). La nota bibliografica che, in appendice, correda il testo contiene i fondamentali riferimenti storiografici. 
ABSTRACT. - Beccaria between criminal law and public economics.

As Mario Romani wrote in 1966: 'the most mature and solid part of Beccaria's work as system builder, that is, his lectures on economia pubblica delivered between 1769 and 1772, and the empirical investigations or consulte stemming from inquiries undertaken in his capacity as a member of government bodies, was for a long time wholly or partly overlooked, or entrusted to unreliable editors, who were ready to print manuscripts the Author himself had not deemed worthy of publication'.

This discomfort is no longer justified. The Elementi di economia pubblica will soon be published, while the Atti di Governo have recently appeared in print. We now know the details of Beccaria's activity in the Supremo Consiglio d'Economia, as Head of the First and then of the Second Department, and as a member of the Consiglio di Governo. We can evaluate his contribution to both theory and practice in the fields of criminal law and economics. The aim of this essay is to assess the consistency between Beccaria's theoretical writings and his field work, with particular reference to freedom of trade (of grains in particular), free bakery, hunting, forest and mines, the Pizzighettone prison, and the Milan house of correction.

Scriveva Melchiorre Gioia nel 1802: "Allorché Beccaria e Verri dicevano delle grandi verità contrarie ai pregiudizi dominanti, senza acquistarsi la taccia di teste calde e di ribelli (spesso unica risposta dell'ignoranza potente), i danni della meta furono messi in tale evidenza" che tra il 1780 e il 1785 il sistema su di essa fondato fu levato in diverse città lombarde. Ma poi “i cangiamenti [...] de' tempi e de' governi, l'ostinazione de' corpi civici, l'ignoranza del popolo fecero risorgere le false inveterate abitudini, e la debolezza cedette ciò che aveva guadagnato la filosofia". ${ }^{1}$ Carlo Cattaneo, dal canto suo, nel ricordare con evidente compiacimento che alcuni anni prima del famoso carcere di Gand, Milano aveva esperimentato la cella segregante come strumento applicabile non solo a fine di lieve correzione, ma anche ai delitti, quale pena severa e rigorosa, sottolineava: "ma qual meraviglia che questi sagaci pensieri nascessero prima che altrove in quel luogo dove Beccaria non solo era scrittore, non solo porgeva publico insegnamento di scienze sociali, ma sedeva autorevole nei consigli dello Stato?". 'Si trattava di un esperimento "ben degno del luogo e del tempo, ove

1 M. GIOIA, Sul commercio de' commestibili e caro prezzo del vitto (1802), in Dettati politici, filosofici, statistici tratti dalle opere minori di Melchiorre Gioia, volume primo Lugano 1850, pp. 303-547, sul punto p. 390 s.

2 C. CatTANeO, Notizie naturali e civili su la Lombardia. Introduzione di M. 
Beccaria scriveva dei delitti e delle pene, e dove magistrati filosofi, come Neri, Carli, Pecci, Verri, e Beccaria stesso, con una vasta riforma, che abbracciava ad un tempo le monete, le imposte, i conti publici, le communi rurali, le manimorte, le strade, i giudizii, le prigioni, le scuole, fondavano la successiva prosperità di questo paese. [...] Onore e gratitudine alla loro memoria!". ${ }^{3}$ Mi paiono citazioni, queste, significative non solo per la loro immediata evidenza, ma anche perché fanno riferimento, con i loro autori e i personaggi richiamati, a quel movimento di pensiero e di azione così felicemente colto e inquadrato da Quadrio Curzio ed altrettanto felicemente espresso con la locuzione "paradigma lombardo", che investì il "campo della storia, della filosofia, della politica, del diritto, dell'economia e, non certo in subordine, della tecnologia" [Quadrio Curzio, 2002, pp. XIV-XVI].

Funzione delle scienze di pubblica economia - si ricordi che l'economia pubblica è definita come "l'arte di conservare ed accrescere le ricchezze in una nazione, e di farne il miglior uso", nonché come "l'arte di fornire con pace e sicurezza non solamente le cose necessarie, ma ancora le comode alla moltitudine riunita" - è quella di suggerire "i mezzi di riunire l'utilità propria con quella del pubblico" in modo tale che "[...] non ci consideriamo più come parti isolate, ma come figli della società, delle leggi e del sovrano; la sfera de' nostri sentimenti diventa più grande e più viva; le passioni esclusive si diminuiscono; le affezioni sociali si dilatano e si inforzano pel potere dell'immaginazione e dell'abitudine; e misurando gli oggetti nelle vere loro dimensioni ci allontaniamo da ogni bassezza e viltà, vizi che nascono sempre dalla falsa misura delle cose". 5 Interesse privato e interesse pubblico, utilità

Talamona. A cura di F. Livorsi e R. Ghiringhelli. Presentazione di E.A. Albertoni in La Lombardia di Carlo Cattaneo, II, Milano 2001, p. 126.

3 C. Cattaneo, Di varie opere sulla riforma delle carceri, in ID., Il Politecnico 1839-1844, a cura di L. AMBROSOLI, Torino 1989, pp. 515-555, in particolare p. 524.

4 C. BECCARIA, Elementi di economia pubblica (d'ora in avanti Elementi), in Scrittori classici italiani di economia politica, parte moderna, tomo XI, Milano 1804, p. I Principj e viste generali, $\mathbb{S}$ I, p. 19. Questo tomo comprende le parti I-III e il cap. I della p. IV, i cui capp. II-IX sono ricompresi nel tomo XII (vedi nt. 5).

5 C. BECCARIA, Prolusione letta il giorno 9 gennajo 1769 nell'apertura della nuova cattedra di scienze camerali nelle Scuole Palatine di Milano da Cesare Beccaria (d'ora innanzi Prolusione), in Scrittori classici italiani di economia politica, parte moderna, tomo XII, Milano 1804, pp. 167-190, in particolare, p. 176. 
propria e utilità del pubblico, dunque, che debbono combinarsi tra di loro, amalgamarsi, ovvero gli interessi privati e le utilità particolari debbono riunirsi tra di loro nell'interesse pubblico, nell'utilità collettiva perché il fine del bene pubblico, che per Beccaria consiste ne "la massima felicità divisa nel maggior numero", 6 possa essere conseguito.

Nello svolgimento e nel compimento di questo processo, Beccaria ripone grande fiducia nella libertà, come forza equilibratrice, quella libertà che "equilibra più d'ogni altra forza gl'interessi degli uomini". Ancora, Beccaria negli Elementi: "Generalmente la libertà assoluta, ossia il non sistema è il migliore di tutti i sistemi che in materia d'annona si possano immaginare dal più raffinato politico". ${ }^{8}$ Con l'immediato riscontro nel Beccaria del 1789, quale consigliere risolutamente avverso alle penali e alle perquisizioni "sempre contrarie allo spirito d'industria che esige libertà e facilità".?

Ma prepotente è la forza egoistica dell'interesse individuale: "Tutte le idee di morale e di virtù, $[\ldots]$ ad ogni minimo vento svaniscono nell'animo umano" ${ }^{10}$ E nel caso in cui il minimo vento minacciasse di mortificare o abbattere le idee di morale e di virtù, si rende necessario l'intervento della legge che freni "l'interesse personale tra quei limiti ne' quali divenga una forza combinata con il bene pubblico, non una contraria e distruttiva di quello". ${ }^{11}$ Funzione della legge è quella di "prevenire quei

6 C. BeCCARIA, Dei delitti e delle pene, a cura di G. Francioni, vol. I dell'Edizione Nazionale delle Opere diretta da L. FIRPO, Milano 1984, Introduzione, p. 23; $\mathbb{S}$ XL False idee di utilità, p.120. Ma v. anche ID., Frammenti sugli odori, in Il Caffè ossia brevi e vari discorsi distribuiti in fogli periodici dal giugno 1764 a tutto maggio 1765, tomo I, Brescia 1765, foglio IV (ristampa a cura di S. RomagnOLI, Milano, 1960), p. 35 (p. 43, nell'ed. Torino 1993 a cura di S. Romagnoli e G. FranCIONI), ove il bene pubblico è identificato "nella massima somma di piaceri, divisa egualmente nel massimo numero d'uomini" [Neppi Modona, 1990, p. 85 s.; j196, 2000, pp. 82-84]. Il testo si legge anche in C. BECCARIA, Scritti filosofici e letterari a cura di L. FIRPO, G. FRANCIONI e G. GASPARI, I Articoli tratti dal Caffè, a cura di L. FIRPO, vol. II dell'Edizione Nazionale delle Opere diretta da L. Firpo, Milano 1984, pp. 23-31, p. 26 per il punto considerato.

7 C. BECCARIA, Elementi [nt. 4], p. II, cap. IV, $\$ 32$, p. 166.

8 Ibid., p. II, cap. V, $\$$ 46, p. 196 s.

9 C. BeCCARIA, Atti di Governo (serie VI: 1789), a cura di R. CANETTA, vol. XI dell'Edizione Nazionale delle Opere diretta da L. Firpo e G. FranCIOnI, Milano 2004 (d'ora in avanti, BeCCARIA, Atti di Governo, XI), doc. 3302, 26 ottobre 1789, p. 707.

10 BeCCARIA, Dei delitti e delle pene [nt. 6], $₫$ XXXVI Della taglia, p. 113.

11 BECCARIA, Elementi [nt. 4], p. II, cap. V, $\$ 48$, p. 199. 
disordini che tendono alla disamalgamazione dell'interesse privato dal pubblico; [essa] deve aspettare quando nascono a rimediare a quelli che turbano gli interessi privati senza fare i pubblici". ${ }^{12}$

In materia di grani, nel cui commercio auspicabile è la libertà assoluta, l'intervento della legge ha ragion d'essere solo quando la libertà assoluta risulti dannosa, ma le modificazioni di essa debbono discostarsi il meno che sia possibile dalla libertà medesima. ${ }^{13} \mathrm{Se}$ "l'autorità pubblica deve essere tutrice del popolo, e con vigorosa fermezza penetrare e dissipare i tenebrosi raggiri dell'imperturbabile avidità di guadagno" ${ }^{14}$ il legislatore, configurato come saggio architetto, che deve far sorgere il suo edificio sull'amor proprio, convinto che l'interesse generale sia il risultato degl'interessi di ciascuno - si ricordi che nel Dei delitti e delle pene Beccaria sostiene che l'autonomia, l'iniziativa individuale, in cui si manifesta la libertà, ove siano fondate sulla considerazione del sociale, hanno gran peso nell'amalgamare gli interessi dei singoli, che, non privati della loro individualità, formano l'interesse pubblico, sulla cui base soltanto si edifica la vera utilità comune ("è falsa idea d'utilità quella che, sacrificando la cosa al nome, divide il ben pubblico dal bene di tutt'i particolari" $)^{15}$ - ebbene, il legislatore, che appunto sia saggio architetto, nel senso ora detto, non sarà costretto con leggi penali e rimedi tumultuosi a separare ad ogni momento il ben pubblico dal bene dei particolari e ad alzare il simulacro della salute pubblica sul timore e sulla diffidenza. Nel Dei delitti e delle pene Beccaria ricorre ancora all'immagine dell'architetto che erige il suo edificio: "[...] il legislatore fa come l'abile architetto di cui l'officio è di opporsi alle direzioni rovinose della gravità e di far conspirare quelle che contribuiscono alla forza dell'edificio". ${ }^{16}$

La legge, dunque, in funzione di garanzia della libertà, della quale deve riconoscere la capacità di conseguire il pubblico bene. Scrive Beccaria negli Elementi: "Tutto deve essere diretto da questa massima

12 C. BeCCARIA, Pensieri staccati, in Scritti filosofici e letterari [nt. 6], III Scritti e frammenti a cura di G. FRANCIONI e G. GASPARI, pensiero XXI, p. 281.

13 BeCCARIA, Elementi [nt. 4], p. II, cap. V, $\mathbb{\$}$ 46, p. 197.

14 Ibid., cap. V, $\$ 63$, p. 220 s.

15 BeCCARIA, Dei delitti e delle pene [nt. 6], $\$$ XL False idee di utilità, p. 120

16 Ibid., $\mathbb{S}$ XLVI Delle grazie, p. 128; $\mathbb{\$}$ VI Proporzione fra i delitti e le pene, p. 41 s. [Cattaneo, 1997, p. 86]. 
che non ha eccezione, cioè che le restrizioni alla libertà non debbono essere poste per l'amore della perfezione ma soltanto per esigenza della necessità, non per far meglio ma per trattenere un disordine" ${ }^{17} \mathrm{Ma}$ quali sono i caratteri della legge elaborata da un saggio, abile architetto? Li indico in modo schematico senza indulgere ad illustrare teoriche, per poi vedere se, come, in quali occasioni abbiano avuto, o no, concreta attuazione. Beccaria esprime propensione per leggi precise, semplici, generali, uniformi, chiare e brevi; avversione per quelle rigorose, complicate, minute, austere, eccessivamente vincolanti. Domina inoltre, anche qui, il fondamentale binomio giustizia-necessità: la legge, come del resto la pena, deve essere giusta e necessaria, è giusta, perché necessaria e, in quanto tale, utile. ${ }^{18}$

Ora, immergiamoci nella realtà delle cose, nella prassi. Giovanni Angelo Sormani chiede una gratificazione di 100 doppie per irrobustire la fabbrica di coperte di lana che "tiene in Asso". Il vice visitatore generale Besozzi, incaricato di assumere informazioni e di esporre le relative risultanze, riassunti gli antecedenti dell'erezione della fabbrica, fa presente che "in oggi non batte che un telaio per alcuni mesi dell'anno", come conseguenza del piccolo capitale disponibile. Ciò nonostante, "per la buona qualità delle coperte di lana che fabbrica, lo smer-

17 BeCCARIA, Elementi [nt. 4], p. III, cap. II, \$17, p. 288 s., nonché p. II, cap. $\mathrm{V}, \mathbb{S} 63$, p. 223 s., in ordine alla panificazione.

18 Occorre disporre per legge ciò che "si crederà indispensabile di prescrivere direttamente. Dico indispensabile perché non le molte e minute, ma le poche e necessarie leggi son quelle che sono osservate" (BECCARIA, Atti di Governo, XI [nt. 9], doc. 3302, 26 ottobre 1789, p. 710). Nel voto espresso dalla corrente, rimasta in minoranza, nella Commissione istituita da Leopoldo II per la riforma del sistema criminale lombardo (infra, nota 128), la prima delle tre argomentazioni svolte contro la pena di morte suona così: "Primo, perché non è giusta, non essendo necessaria [...]". Si doveva ricorrere piuttosto alla pena dei lavori pubblici, che "può rendersi efficacissima ed utile allo scopo d'ogni buona legislazione criminale, che è prima la correzione del reo" (C. BECCARIA, Atti di Governo (serie XI: gennaio-dicembre 1794), a cura di R. CANETTA, II (settembre-dicembre 1794), vol. XVI dell'Edizione Nazionale delle Opere diretta da L. Firpo e G. Francioni, Milano 2009 (d'ora in avanti BeCCARIA, Atti di Governo, XVIII). Appendice Voto degli infrascritti individui della Giunta delegata per la riforma del sistema criminale nella Lombardia austriaca risguardante la pena di morte (24 gennaio 1792), pp. 965-972, sul punto, pp. 968, 972. Il binomio giusto-necessario ripetutamente ricorre nel Dei delitti e delle pene. Se ne vedano [nt. 6] i $\$ \$$ II Diritto di punire, pp. 2932; XXVIII Della pena di morte, pp. 86-95; XXXI Delitti di prova difficile, pp. 100-103. 
cio delle medesime va tutto dì aumentandosi tanto nello Stato che ne' paesi esteri"e, pertanto, va senza dubbio aiutato nella sua intrapresa. Non però con gratificazioni: "le picciole gratificazioni che di tanto in tanto si sono accordate a consimili fabbriche, a poco o a nulla hanno giovato alla loro prosperità, poiché i graziati se ne valgono per lo più ne' loro pressanti bisogni”. Di gran lunga preferibile è assistere il Sormani "con una sovvenzione di L. 10.000 per anni dodeci, senza interesse [...]". Il che lo porrebbe nella situazione "d'avere due telari battenti di coperte, li quali in un anno possono dare n. 1.900 coperte, che diminuirebbero la passività dello Stato in questa parte [...]". Condizioni proposte e necessarie: idonea sicurtà, obbligo di formare due allievi nazionali, e di tenere sempre battenti due telai. E', questo, anche l'intendimento di Beccaria, che tiene pure presente "la vantaggiosa sittuazione della Vallassina per il mantenimento delle pecore, che danno la materia prima di questa fabbrica $[\ldots]]^{\prime 19}$

Nell'affrontare la molteplicità dei casi, che la realtà gli sottopone -non è possibile qui altro fare che procedere per esempi- l'impostazione che Beccaria intende seguire, e che in effetti segue, è chiara. Nel rivolgersi a Kaunitz, che gli aveva "recato le massime dettate e prescritte dalla sapienza di Sua Maestà l'augusto padrone, a regola delle sovvenzioni ed aiuti per animare i commercianti ed ampliare il commercio", Beccaria tiene ben presente che due sono le massime volte "ad introdurre commercio e manifatture dove non ve ne sieno"; a "dilatarle e perfezionarle dove vi sono". "Il procurar ciò è d'avere quello che non s'ha e che realmente giovi d'avere, ed il perfezionare ed ampliare quello che s'ha, e che in effetto giovi di ampliare". Per soddisfare la prima, ecco le sovvenzioni, in quanto "il solo caso della prima le richiede e le merita [...]". Per soddisfare la seconda, ecco i premi, "i quali veramente sono i soli che a proposito distribuiti, possono animare la industria domestica in quello che s'ha". A queste massime, stabilite da

19 C. BeCCARIA, Atti di Governo (serie III: 1784-1786) a cura di R. CANETTA, vol. VIII dell'Edizione nazionale delle Opere, diretta da L. FirPo e G. FrAnCIONI, Milano 1993 (d'ora in avanti, BeCCARIA Atti di Governo, VIII), doc. 1209, 19 agosto 1785, p. 485 s., ma v. anche C. BECCARIA, Atti di Governo (serie IV: 1784-1786) a cura di R. CANETTA, vol. IX dell'Edizione Nazionale delle Opere, diretta da L. FIRPO e G. FranCIONI, Milano 1998 (d'ora in avanti BeCCARIA, Atti di Governo, IX), doc. 1689, Attività del Dipartimento III nel 1786 (relazione, 31 marzo 1787), pp. 226 s., 244. 
Sua Maestà, Beccaria intende conformarsi con la "più impegnata attenzione e vigilanza". ${ }^{20}$

Assai complessa era la situazione, meglio la richiesta avanzata dal lanificio Guaita: chiedeva il pagamento delle esenzioni daziarie arretrate a far tempo dal 1770 per un ammontare di circa 30.000 lire, nonché una somministrazione di 100.000 lire senza interessi. La somministrazione, che Beccaria tiene ben distinta dalla sovvenzione -le sovvenzioni talora impropriamente recano questo nome "poiché non tendono direttamente al promovimento del commercio, ma servono di ripiego alla Regia Camera per porre in circolazione la moneta di rame, dandosi questa con idonea sicurtà a $1 / 2 \%$, per essere restituita dopo un anno in moneta nobile [...]", ${ }^{21}$ consisteva dunque, nel caso, nella dazione della somma indicata in monete di rame, che dovevano essere restituite nel giro di dieci anni, ripeto, senza interesse alcuno, mentre in genere, tiene a precisare Beccaria l'interesse variava dall' $1 \frac{1}{2} \%$ al 3 e $1 / 2 \%$. Ad un rilievo formulato dalla Corte (il rilievo, che riguardava la locuzione "solito interesse", riferito a quello dell' 1 e $1 \frac{1}{2} \%$, era fondato sul fatto che "l'Imperatore vuole che [tale interesse] sia fissato al 3 e $1 / 2 \%$, eccetto il primo anno"), Beccaria rispondeva che l'interesse dell'1 e $1 \frac{1}{2} \%$ era definito "solito" non già con riferimento "alle sovvenzioni che si fanno dal fondo del Commercio ["uno fra i diversi strumenti - né l'unico sotto l'aspetto finanziario - a disposizione dei governanti per intervenire, in particolare, nell'attività manifatturiera" [Trezzi, 1982, p. 175]", quanto piuttosto "alle somministrazioni che si fanno in moneta di rame, per essere restituita in moneta nobile [...]". ${ }^{22}$ Ritornando al lanificio Guaita, della sua richiesta veniva interessato il

20 BeCCARIA Atti di Governo, VIII [nt. 19], doc. 1421, 2 ottobre 1786, p. 837 s.

21 Ibid., p. 838.

22 BeCCARIA, Atti di Governo, IX [nt. 19], doc. 1814, 11 giugno 1787, p. 434 s. Le risorse del fondo del Commercio potevano avere, per Beccaria, due applicazioni: "[...] I $\mathrm{I}^{\circ}$ sovvenire le nuove e veramente importanti manifatture coll'accordare all'intrapenditori che faranno con fondamento sperare di saperle sostenere soccorsi in denaro con sicurtà anche bancarie coll'interesse del $3 \frac{1}{2} \%$, dopo un anno gratuito; $2^{\circ}$ di premiare quelli che migliorano e aumentano le già esistenti, in proporzione della importanza e quantità di questa miglioria. Quando i progetti erano diversi in qualche aspetto da ciò che si esige con queste massime, e che nondimeno si è creduto conveniente di non rigettarli, si è ricorso allo spediente delle somministrazione in moneta di rame [...]" (ibid., doc. 1689, Attività del Dipartimento III nel 1786 (relazione, 31 marzo 1787), p. 245). 
Procuratore Generale affinché si pronunciasse in merito. Il lanificio chiedeva inoltre che venissero aumentati, e in modo sensibile, "i dazi d'entrata de' panni forestieri”. Beccaria è per la negativa. L'aumento doveva essere di notevole entità perché ne ritraessero effettivo vantaggio le manifatture nazionali, le quali erano poco numerose. V'era pertanto da temere che in realtà "l'aumento [...] si risolva in un aggravio per il pubblico, che deve ad ogni modo provvedersi dall'estero della maggior parte di queste". Da ultimo, ecco la richiesta di un qualche "sussidio" per potersi provvedere dall'estero di un arrotino per le forbici che servono alla cimatura dei panni e di un "fabbricatore di scardassi". Di costoro lo Stato è privo e del loro acquisto avrebbe potuto avvantaggiarsi non solo il lanificio, ma, per l'appunto, l'intero Stato. L'affare non appariva a Beccaria di gran momento, però pensava che la "graziosa concessione" di 100.000 lire per dieci anni, ove fosse andata a buon fine, doveva mettere in grado il lanificio di provvedere a sé di per se stesso senza ulteriore aggravio per il fondo del Commercio. Termina il suo voto con una riflessione, che può valere come massima: "quando le manifatture non possono sostentarsi che con continue largizioni del Regio Erario, vi è sempre da temerne la pronta decadenza, invece di uno stabile incremento". ${ }^{23}$

A interventi di sussidio per la propria attività manifatturiera era interessata la fabbrica milanese Gattoni che, in aggiunta ad altre provvidenze, aveva chiesto un prestito di 40.000 lire in moneta di rame da restituire in moneta nobile in quattro anni all' $1 \frac{1 / 2}{2} \%$. Secondo Beccaria

23 BeCCARIA, Atti di Governo, VIII [nt. 19], doc. 1436, 12 ottobre 1786, pp. 853-856. Il voto, per quanto riguarda l'arrotino, non sembra congruente con quanto si legge in BeCCARIA, Atti di Governo, IX [nt. 19], doc. 1689 Attività del Dipartimento III nel 1786 (relazione, 31 marzo 1787), p. 228. E' opportuno rilevare come, dopo aver interloquito a Como sul finire del 1787 con la Congregazione municipale, con l'Intendenza politica e con la Camera mercantile e dopo aver visitato alcune fabbriche ivi operanti, Beccaria avesse espresso un giudizio negativo sul livello qualitativo del prodotto delle manifatture comasche ("Generalmente, la fabbricazione delle stoffe è assai cattiva in Como, e per il lustro e per la disuguale tessitura, e queste stoffe si riducono presso che quasi da pertuto ai soli mantini, li amoere; i lustrini sono spariti dai telai; alcuni pochi rasi rigati e sogli si fanno ancora [...]" [Caizzi, 1968, p. 118]. Non mancavano però le eccezioni, tra queste il lanificio Guaita, "che è il solo lanificio di Como ed il più considerabile dei pochi esistenti nella Lombardia Austriaca" (BECCARIA, Atti di Governo, IX [nt. 19], doc. 2140, 3 e 17 dicembre 1787 (relazione e voto), pp. 854 864, sul punto, p. 861 s.). In ogni caso "[...] i Guaita ebbero alti e bassi, ma non riuscirono mai a fare a meno degli aiuti del Governo [...]” [Capra, 1984, p. 474]. 
"[...] l'obbligare per patto la Camera a dare questa somministrazione per quattro anni veste piuttosto l'aria di una sovvenzione che di semplice somministrazione introdotta unicamente per ismaltire la moneta di rame, allorché stagna nelle Regie Casse [...]”. E poi, sotto questo aspetto la manifattura progettata dal Gattoni non poteva essere ricompresa tra "quelle favorite nelle recenti massime di Sua Maestà dateci per norma”. Che il Gattoni, dunque, eriga la sua manifattura e poi potrà chiedere "qualche somministrazione nelle forme regolari, quando le circostanze di questa Cassa lo permettano". ${ }^{24}$

Particolarmente favorevole alle sovvenzioni Beccaria si rivela in ordine alle attività mineralogiche e siderurgiche. Una minuta di consulta di Beccaria (1774) c'informa sulla procedura che si era soliti seguire allorché un privato, scoperta una miniera 'promettente', avesse ritenuto opportuno investirvi danaro e forza di lavoro. Il Magistrato Camerale, pervenuta che sia la supplica dell'interessato, provvede a informare il Podestà competente per territorio -"Giusdicenti dei rispettivi luoghi"affinché assista il richiedente nello svolgimento delle attività necessarie e riferisca "il risultato rimettendo i campioni": ciò a tutela degli interessi della Regia Camera, dei titolari di eventuali diritti dei proprietari dei fondi ove è sita la miniera, ma anche a tutela di chi per avventura fosse stato in precedenza investito di concessione "nel medesimo circondario". La pratica passa poi al Fisco, che, insieme con le parti interessate, provvede alla stesura dei capitoli. Di questi si fa consulta al Governo "per poscia passare sulle superiori deliberazioni all'investitura". ${ }^{25}$

Molte furono le richieste avanzate: tra le tante, ricordo i casi delle miniere e ferriere a Introbio ${ }^{26}$ dei forni e fucine a Begna. ${ }^{27}$

24 BecCaria, Atti di Governo, VIII [nt. 19], doc. 1492, 4 dicembre 1786, pp. 928-930; IX [nt. 19], doc. 1689, Attività del Dipartimento III nel 1786 (relazione, 31 marzo 1787), p. 231.

25 C. BeCCARIA, Atti di Governo (serie I: 1771-1777) a cura di R. CANETTA, vol. VI dell'Edizione Nazionale delle Opere, diretta da L. FIRPO, Milano 1987 (d'ora in avanti, BECCARIA, Atti di Governo, VI), doc. 259, $1^{\circ}$ luglio 1774, p. 435 s. Il 16 luglio Firmian manifestava a Carli la propria approvazione intorno alla procedura adottata dal Magistrato (ibid., doc. 260, p. 437).

26 BeCCARIA, Atti di Governo, VIII [nt.19], doc. 1332, 12 giugno 1786, p. 719; doc. 1349, 10 luglio 1786, p. 739 s.

27 Ibid., doc. 1411, 21 settembre 1786, p. 826; doc. 1471, 20 novembre 1786, p. $897 \mathrm{~s}$. 
Importante era stata l'attività estrattiva dalla cava del Passo, capace di fornire ottimo granito, che poteva sostituirsi a quello estero, nella lastricazione delle strade milanesi. "[...] fu esposto che il presente lastrico della città consistente nelle sole guide era di quadretti 121.500 , il cui valore è di circa centomilla scudi, la qual somma insieme ad altre molto maggiori che si sogliono fare in altre molte opere di migliarolo estero [o miarolo, questo il nome del granito], d'ora in avanti rimarranno nello Stato [...]". Di qui la necessità di "continuare vigorosamente l'incaminamento di quella cava, e procurare le facilitazioni all'introduzione di quel sasso", il che coinvolgeva inoltre il "regolamento delle circostanti acque". Di qui anche la necessità di intervenire con "eccitamenti finanziari" [Frumento, 1963, pp. 314317], ovvero con premi che, nel caso, consistevano in medaglie d'oro, ciascheduna "di zecchini 24". ${ }^{28}$ In questa materia Beccaria trovò un valido collaboratore in padre Ermenegildo Pini -"cuore saggio e intendente, il delegato alle miniere vigilava [...]" [Frumento, 1963, p. 354]-, del quale, oltre alla specifica competenza, commendava l'economia nelle spese e l'esattezza nel darne conto. "Le minute sono da lui giustificate colla citazione anche dei confessi [...] Siccome però alcuni di questi confessi gli sono necessari per il seguito dei conti che tiene con diverse persone, così ho creduto conveniente che gli ritenesse presso di sé". ${ }^{29}$ Una fiducia piena, dunque, che non risultava mortificata dal fatto che, in occasione di un viaggio effettuato per visitare due nuovi forni norvegiani in Valcavargna e in Valsassina, nonché le cave di granito del Passo, aveva presentato una nota spese relativa, in parte, all'oggetto miniere, in parte, all'oggetto strade, le prime rimborsabili dai proprietari dei due forni, che avevano ottenuto la pattuita gratificazione, le seconde dal fondo delle Strade. Sarebbe stato bene, pertanto, che l'esperto minerologo avesse separato questi oggetti "per attribuire la contingente ai rispettivi fondi. Si eccita lo stesso

28 Ibid., doc. 1234, 10 dicembre 1785, p. 547; doc. 1423, 3 ottobre 1786, p. 840.

29 Ibid., doc. 1234, 10 dicembre 1785, p. 548. L'attività estrattiva della cava del Passo aveva confermato la fondatezza delle ottimistiche previsioni. Ed ora, 1787, il Pini avanzava la proposta di aprire nuove cave, per l'estrazione della beola, nei pressi del Lago di Piona. "Non posso che convenire -scrive Beccaria nel suo voto- coi plausibili suggerimenti del Padre Pini, e noi saremo anche per questo titolo debitori al suddetto di una scoperta avvantaggiosa allo Stato, passivo di tutto questo prodotto di tanto uso" (BeCCARIA, Atti di Governo, IX [nt. 19], doc. 1733, 30 aprile 1787, p. 323 s.). 
padre Pini a farlo, per portar poi tutto il risultante alla cognizione del regio Imperial Consiglio". ${ }^{30}$

Rimaniamo ancora nel settore siderurgico. A Francesco Mornico, che intendeva costruire in Valsassina una fucina stiriana - che involgeva "il lavoro per noi nuovo dell'acciaio" ${ }^{31}$ - era stata concessa, su sua richiesta, una sovvenzione di L. 13.000 da convertirsi in gratificazione all'avvio della fucina [Frumento, 1963, p. 335 ss.]. La richiesta originaria, invero, era stata diversa e più complessa: la richiesta ora ricordata si accompagnava, in alternativa a quella che subordinava l'obbligo di erigere la fucina stiriana al prestito di L. 30.000 senza interessi per 16 anni. Fra le due "proposizioni" del Mornico, ragionava Beccaria, la meno onerosa per la Camera era la seconda, in quanto "gli interessi semplici delle 30.000 lire per 16 anni al 3 e $1 / 2 \%$ importano una donazione di 1. 16.800". E, d'altra parte "una gratificazione di L. 13.000 è meno imbarazzante per la regia Camera che un lungo imprestito di L. 30.000". ${ }^{32}$ In modo forse più chiaro Beccaria ribadiva i motivi della sua preferenza. Quando si tratta di "intraprese per le quali non conviene mai perder di vista i molti rischi che corrono gli intraprenditori, il metodo di dare mediocri sovvenzioni gratuite, da convertirsi poi in gratificazioni ad opera compiuta e lavoro incamminato, dentro un breve spazio di tempo, come si è praticato, è più vantaggioso per assicurare l'esito di simili intraprese per l'intraprenditori stessi e per la vera economia del fondo del Commercio, che l'altro di accordare più generose sovvenzioni, le quali non si potrebbe fare a meno di darle a tenui interessi e restituibili dopo lungo tempo".33 Imboccata dunque la strada preferita da Beccaria, non rimaneva al Mornico che fornire idonea sicurtà "per liberarsi dall'obbligo della restituzione" di quanto ricevuto a titolo di sovvenzione, in caso di inadempimento dell'obbligo assunto. Ritenuta idonea la sicurtà indicata nella persona di Massimiliano Manzoni, "proprietario della miniera di ferro del monte Artino in Valsassina, ${ }^{34}$ Beccaria si dimostra non convinto dell'opportunità di un patto che, inserito nel pro-

30 BeCCARIA, Atti di Governo, VIII [nt.19], doc. 1530, 27 dicembre 1786, p. 973.

31 BeCCARIA, Atti di Governo, IX [nt. 19], doc. 1689, Attività del Dipartimento III nel 1786 (relazione, 31 marzo 1787), pp. 255-257.

32 BeCCARIA, Atti di Governo, VIII [nt. 19], doc. 1390, 31 agosto 1786, pp. 795-800

33 BeCCaria, Atti di Governo, IX [nt. 19], doc. 1689, Attività del Dipartimento III nel 1786 (relazione, 31 marzo 1787), p. 254 s.

34 Ibid., doc. 1774, 21 maggio 1787, p. 392. 
getto, prevedeva la possibilità per il Mornico, di "mutarla [la fucina stiriana] dopo un anno, ancorché riuscisse felicemente". Si tratta di un patto che a Beccaria risultava "per lo meno inutile e lo direi sospetto, se non si trattasse del Mornico, onest'uomo, e di un mediatore come il Padre Pini". ${ }^{35}$ La conclusione? "Conviene cancellarlo", questo patto. Si era nel febbraio 1787. Qualche mese dopo pervengono le osservazioni della Corte: rivolto l'invito a "concedere con più accortezza le sovvenzioni per la costruzione di forni", approvava che la "la sovvenzione, sia tramutata in premio". Beccaria, nel dare riscontro a tali osservazioni, risponde in un modo, che sotto il profilo tecnico, non risulta limpido. Certo, era conveniente incoraggiare quegli imprenditori, che intendevano correre il rischio dell'intrapresa volta a "introdurre la costruzione dei forni" così importante perché producono "una maggior quantità di ferro in minor tempo, con il risparmio di un quarto del carbone", ma altrettanto certo era che dopo che "l'esperienza ha convinto ognuno, non deve più esservi luogo a gratificazioni per quest'oggetto". ${ }^{36}$ Ma per altri oggetti, invece, sì. Tal Pietro Ferriù risultava per Beccaria meritevole dei "trecento scudi proposti in favore di un introduttore di una fabrica nuova ed utile per il paese, e di dargli i mezzi di o perfezionarla o dilatarla". Occorreva comunque prudenza: i due terzi della somma avrebbero dovuto essere depositati presso la Camera mercantile perché fossero convertiti a beneficio della fabbrica stessa, provvedendola degli utensili e delle scorte necessari. L'altro terzo poteva essere lasciato alla libera disponibilità dell'interessato "per incoraggirlo e supplire a quei urgenti bisogni in cui deve ritrovarsi un onesto, abile ma povero artefice". Era anche il caso di tal Monfardini, che chiedeva una gratificazione per aver ottenuto una vernice oleosa per carrozze, simile alla vernice di Parma. La Camera mercantile, interpellata, è d'accordo sulla gratificazione, ma non solo: era opportuno che la Reale Accademia lo premiasse con medaglia d'oro. Similmente Beccaria, che, favorevolissimo circa la gratificazione, "trattandosi di un discretissimo premio di lire 200 di Milano", sorvola in ordine alla medaglia. ${ }^{37}$

35 Ibid., doc. 1613, 12 febbraio 1787, p. 122 s.

36 Ibid., doc. 2005, 24 settembre 1787, p. 686 s.

37 C. BECCARIA, Atti di Governo (serie V: 1788), a cura di R. CANETTA, vol. X dell'Edizione Nazionale delle Opere diretta da L. Firpo e G. Francioni, Milano 2000 (d'ora in avanti, BeCCARIA, Atti di Governo, X), doc. 2633, I ${ }^{\circ}$ settembre 1788, p. 558; doc. 2295, 17 marzo 1788, p. 161. 
Anche in prosieguo di tempo, non mancheranno rilievi da parte della Corte circa l'opportunità delle sovvenzioni, anche sotto forma di dazione di monete erose. Così, per esempio, data la difficoltà in cui si trovano le già esistenti fabbriche di Como, risulta inopportuno concedere sovvenzioni a nuovi fabbricanti; troppe sono state le sovvenzioni concesse ai setaioli, mentre sarebbe bene, piuttosto, concedere sovvenzioni alle manifatture da promuovere, tra le quali la filatura della seta non può di certo essere annoverata. Ancora, stanno bene le sovvenzioni, ma non nella straordinaria misura che si è raggiunta nel 1787; non pochi sono i beneficiari che non adempiono le condizioni per la restituzione dei capitali ricevuti. Insomma, "le sovvenzioni debbono essere considerate una sorta di premio per gli intraprenditori che adottano nuovi metodi di lavorazione" e il Consiglio di Governo dovrebbe essere sollecito nell'individuare tali manifatture. ${ }^{38}$

Circa la situazione comasca, sulle cui difficili vicende fu spesso chiamato ad intervenire [Caizzi, 1957, pp. 24-36; Capra, 1984, pp. 575 577], Beccaria notava come nella disastrosa annata 1787 la manifattura serica si era sostenuta "col lavorerio di speculazione, affine di mantenere i più abili operai", occupati gli altri nella filatura e nella tessitura del lino oppure nel lavoro manuale di bonifica (il prato Pasquè), per correggerne la malsania [...]". Fattosi ora il raccolto dei bozzoli del baco da seta ("galette") e, quindi, tornati tessitori e filatori al loro dismesso lavoro, il negare somministrazioni in monete di rame a chi le chiede al fine di ampliare il proprio lavoro potrebbe produrre "sconcerti" per i molti operai che rimarrebbero oziosi. Inoltre la generalizzata negazione di tali sovvenzioni, ovvero, meglio, di tali somministrazioni in moneta erosa prive-

38 Ibid., doc. 2718, 6 ottobre 1788, p. 663 s.; doc. 259511 agosto 1788, pp. 513 515. Due anni dopo, nel 1790, avendo sotto gli occhi "il bilancio del fondo di commercio relativo all'anno medesimo" e rilevando che esso presentava un notevole avanzo, il Kaunitz sollecitava l'impiego del fondo stesso, dal momento che le "circostanze della Lombardia rendono necessaria una efficace spinta per promuovervi diversi rami di manifattura suscettibili di maggior incremento, tanto per diminuire in alcuni la passività del Paese, che per portarne altri alla attività [...]”. E terminava i suoi rilievi con questa considerazione: "[...] sarà sempre preferibile alla cura di economizzare su di un fondo che ha la fissata sua destinazione, quella di farne un buon uso, combinandone le misure coi riguardi dovuti all'agricoltura, e l'incoraggiamento dell'industria d'ogni genere purché sia ben assortita alla condizione del paese”. Cito da Romani, 1977, p. 201 s. e nota 203. 
rebbe "l'Erario e il pubblico dei vantaggi" - ché indubbiamente di vantaggi per il Governo si tratta - connessi all'uso "di mettere in circolazione la giacente moneta di rame [...] e di animare le manifatture, specialmente del nazionale prodotto delle sete". Già, vantaggio dell'Erario, ribadirà Beccaria, secondo un' argomentazione della quale anche in prosieguo di tempo si avvarrà: "[...] perché si è applicato impropriamente il nome di sovvenzione alle somministrazioni in moneta di rame, che non servono direttamente al promovimento delle manifatture, ma sono un ripiego per mettere in circolazione la moneta erosa". ${ }^{39} \mathrm{Si}$ tratta di un'argomentazione che Beccaria aveva già svolta in risposta ad una richiesta di sovvenzione da parte della milanese fabbrica Gattoni, in ordine alla quale si era deviato dalle regole per varie considerazioni, tra le quali, questa: si era concesso "l'uso della casa all'acquirente prima della stesura del contratto". Beccaria specifica: "si trattava di luogo vuoto e che esiggeva considerabili adattamenti dall'essere di ergastolo a quello di fabbrica per parte dell'acquirente" ${ }^{40}$ Tutto sommato si trattava di un buon affare. L'edificio era di vaste dimensioni, "ma [...] per la sua costruzione e situazione era di difficile vendita, né mai certamente per il prezzo che era costato alla Regia Camera, fu venduto per mille lire di più della stima dell'ingegnere camerale Giussani, con l'obbligo al compratore di assumersi il livello di cui era gravato il detto sito [...]". ${ }^{41}$ Ciò poneva particolari problemi circa la natura della sicurtà prestata e della sua idoneità.

Con la vicenda Gattoni, che ora abbandono, siamo posti a contatto, o meglio, immessi nella realtà carceraria della Lombardia austria-

39 BeCCARIA, Atti di Governo, X [nt. 37], doc. 2718, 6 ottobre 1788, p. 663 s.; doc. 2503, 30 giugno 1788, p. 405 s. In merito all'impiego dei disoccupati nel "lavoro manuale sul prato Pasquè per correggere la malsania di quel luogo troppo vicino alla città", v. infra, nota 135. Per un caso di sovvenzione, che in realtà è somministrazione, si veda quello dei fratelli Campioni, impegnati nella costruzione di impianti per la raffinazione del ferro e per i quali si prevedeva anche "un donativo di L. 5.200, donativo che può contemplarsi per una vera gratificazione" (BECCARIA, Atti di Governo, IX [nt. 19], doc. 1584, 29 gennaio 1787 , pp. 83-85).

40 BeCCARIA, Atti di Governo, X [nt. 37], doc. 2532, 14 luglio 1788, p. 438.

41 BeCCARIA, Atti di Governo, IX [nt. 19], doc. 1689, Attività del Dipartimento III nel 1786 (relazione, 31 marzo 1787), p. 231. Era risultata accettabile la proposta d'acquisto libero fuori d'asta di lire 27.550, “coll'obbligo all'aquirente di assumere sopra di sé il canone di cui è gravato il luogo verso il Luogo Pio Trivulzi di L. 750". BECCARIA, Atti di Governo, VIII [not. 19], doc. 1492, 4 dicembre 1786, pp. 928-930. 
ca della seconda metà del Settecento, nel caso, in particolare, con l'Ergastolo di Milano, che veniva ora dismesso e sul quale aveva posto gli occhi Gattoni per stabilirvi la sua manifattura e con l'Ergastolo di Pizzighettone che accoglierà d'ora in poi parte di coloro che, a vario titolo, erano incappati nelle maglie della giustizia. Beccaria fu ripetutamente chiamato a fare fronte alle tante questioni che le due istituzioni, insieme con la Casa di correzione, lo si può ben dire, quotidianamente proponevano. Era una realtà che aveva attratto e al contempo preoccupato anche Leopoldo II. A Pizzighettone v'era l'Ergastolo, dove i condannati a morte, che al tempo (1791) erano otto, conducevano una vita tale da far apparire la morte come una liberazione. "Sempre incatenati al pancone, non hanno che pane e acqua il che fa che sono sempre malati". A costoro andrebbe accordato di prendere qualche volta l'aria altrimenti muoiono di malattia, rilevava e suggeriva Leopoldo. Migliori erano le condizioni dei condannati a tempo, al momento in numero di "400 sotto la direzione di un Sopraintendente e di 40 Esecutori. I condannati s'impiegavano ai lavori delle fortificazioni o alla campagna, o a tessere, o filar cotone. Non hanno altro che del cattivo di munizione, e acqua, il resto bisogna se lo guadagnino col lavoro; sono ai ferri, ma alloggiano in certe casematte buone, comode, sane e ariose, e molto forti e ben custodite. Vi sono tutti i comodi necessari, hanno dei panconi per dormire con degli strapunti". Quanto alle donne esse sono "17 in una stanza comoda, con buoni letti, ben vestite, e forse troppo ben trattate". ${ }^{42}$

Lo preoccupava anche lo stato dell'Ufficio di Polizia: "Le cose di pulizia non si fanno punto, neppure le cose pettorali [Gorla, 1981, 584-588; Pansini, 1986, p. 10 s.] che si mandano al tribunale di prima istanza o collegialmente". ${ }^{43}$ Ecco i provvedimenti. Quanto all'Ergastolo di Pizzighettone, richiesta la lista dei detenuti e ricevuta tosto la nota dei carcerati insieme con le osservazioni del Direttore e le riforme auspicabili - una sorta di Rapporto generale che 'fotografa-

42 Osservazioni di Sua Maestà l'Imperatore sulla Lombardia (d'ora innanzi Osservazioni leopoldine), in Haus-Hof-und Staatsarchiv, Wien (HHSAW), Familien Archiv, Sammelbände, Karton 21, p. 67 s. Si tratta della relazione, contenente una corposa serie di osservazioni, giudizi, impressioni e valutazioni, dettata da Leopoldo al segretario Stefani in occasione del viaggio in Lombardia dei mesi di maggio e giugno 1791.

43 Ibid., p. 152. 
va' la situazione "al dì 24 maggio 1791", opera di Michele Donadeo, ${ }^{44}$ - era disposta una serie di provvidenze a favore dei condannati, a tempo ed a vita: era necessario "di far fare a quell'Ergastolo una Infermeria che non sia come adesso una di quelle prigioni, ma ariosa e ventilata, dove si custodiscano bene i malati, gli si diano lenzuoli, e maggiore nutrimento nel tempo della convalescenza per metterli in forze, si dia ai condannati dei pagliacci fuori che ai bollati e condannati a vita". Questi ultimi "non dovranno più essere nella medesima carcere, ma essere distribuiti uno o due nelle altre carceri con una catena tanto lunga da poter passeggiare, e darli il comodo di poter lavorare per guadagnarsi qualche cosa". ${ }^{45}$ Ricordo soltanto che Beccaria e Morosini, incaricati di dar corso al motu proprio leopoldino, assunte le debite informazioni dall'Ispettore, disposero che "essendo in oggi la rispettiva catena soltanto braccia 4, possa allungarsi altre braccia 10 , ritenuto però che il lucchetto che attualmente si attacca alle balze de' detti condannati per assicurarli mediante la catena affissa al muro, riescendo d'un enorme peso alli stessi condannati, si possa invece attaccare all'anellone conficcato nel muro vicino a terra, e non in alto, cosicché non abbiano i condannati a portare il peso dello stesso lucchetto e possano più comodamente passeggiare" ${ }^{46}$ Beccaria, in rappresentanza del Magistrato Politico Camerale, Morosini, in rappresentanza del Supremo Tribunale di giustizia, insieme con il direttore della Facoltà medica di Pavia Johann Peter Frank, con il regio architetto Piermarini e con il soprintendente alle fabbri-

44 Alla richiesta (ibid., p. 156) si rispose con una Nota dei carcerati nell'Ergastolo di Pizzighettone nel 1785, ed osservazioni del Direttore sopra il medesimo e riforme da farvisi. Allegato n. 27 alle Osservazioni leopoldine [nt. 42], karton 23, pp. 812-817, ov'è anche il Rapporto generale dimostrativo il numero de' condannati al Regio Ergastolo, a firma di Michele Donadeo, relativo "al dì 24 maggio 1791".

45 Ordini diversi dati da Sua Maestà in occasione della sua gita in Lombardia sulle Provincie di Mantova Cremona Lodi Pavia Como, e spediti nel 16 giugno 1791. Allegato n. 9 alle Osservazioni leopoldine [nt. 42], karton 23, p. 538.

46 C. BECCARIA, Per migliorare la sorte dei condannati. 1791, in ID., Opere, a cura di S. RomagnOLI, volume secondo, [Firenze] 1971, doc. n. LVII, 14 settembre 1791, pp. 723-726; ma v. anche C. BECCARIA, Atti di Governo (serie VIII: 1791), a cura di R. CANETTA, vol. XIII dell'Edizione Nazionale delle Opere diretta da L. FIRPO e G. FrAnCIONI (d'ora innanzi, BECCARIA, Atti di Governo, XIII), doc. 4375, 10 ottobre 1791, pp. 562-564. 
che camerali Pertusati furono coinvolti nei non facili e brevi lavori relativi all'infermeria dell'Ergastolo di Pizzighettone. ${ }^{47}$

Ma il disegno di Leopoldo era tanto vasto quanto ambizioso: intendeva porre rimedio alle innumerevoli disfunzioni che affliggevano l'amministrazione della giustizia. Ed era questo il compito affidato alla Giunta istituita con motu proprio sovrano del 16 giugno 1791, della quale faceva parte anche Cesare Beccaria, appositamente incaricata di riformare il sistema criminale lombardo [Cavanna, 1975, pp. 81-85; 2005 , pp. 365-368]. V'era un problema di fondo, cruciale da affrontare: "Quello però che più di tutto potrebbe giovare sarebbe, se si potesse ottenere, la diminuzione dei delitti col prevenire i medesimi, e col rendere più facili i loro scoprimenti. Questi oggetti però della massima importanza, e che sono connessi in gran parte colla costituzione fisica, ed ecomomica dello Stato esiggono un lungo detaglio, ed esame, e si procurerà per quanto è possibile di discuterlo nella Giunta" ${ }^{48}$ Che allo "scoprimento dei delitti" e alla loro repressione andasse affiancata la prevenzione era idea di certo non nuova, un'idea espressa e ribadita più volte nel tempo da Kaunitz e da Firmian: "la frequenza stessa delle pene gravi, usata nello Stato di Milano con poco buon successo sembra vie più giustificare l'osservazione fatta dai giureconsulti li più illuminati, e dai filosofi che il rigore della pena è un riparo debole ai delitti". ${ }^{4}$ Occorreva occuparsi delle minori "delinquenze che conducono, infettando il costume a' delitti gravi”. Specialmente gli oziosi, i ladruncoli, i rei di aggressione, soprattutto se colti in flagrante, dovevano essere, con

47 Ibid., doc. 4142, 18 luglio 1791, p. 274 s.; doc. 4167, 25 luglio 1791, p. 309; doc. 4375, 10 ottobre 1791, pp. 562-564; C. BECCARIA, Atti di Governo (serie IX: 1792) a cura di R. CANETTA, vol. XIV dell'Edizione Nazionale delle Opere diretta da L. FIRPO e G. Francioni, Milano 2007 (d'ora in avanti, Beccaria, Atti di Governo, XIV), doc. 4655, 7 maggio 1792, p. 201; doc. 4751, 9 luglio 1792, pp. 306-308; doc. 4790, 23 luglio 1792, p. 356; doc. 4904, $1^{\circ}$ ottobre 1792, p. 508; C. BECCARIA, Atti di Governo (serie X: 1793) a cura di R. CANETTA, vol. XV dell'Edizione Nazionale delle Opere diretta da L. FirPo e G. Francioni, Milano 2007 (d'ora in avanti, BeCCARIa, Atti di Governo, XV), doc. 5322, 21 maggio 1793, p. 296, documento che, a quanto risulta, è l'ultimo che, tra quelli pubblicati nell'indicata sede, riguardi l'infermeria dell'Ergastolo di Pizzighettone.

48 Oggetti da prendersi in considerazione per la sistemazione delle Preture. Allegato n. 10 alle Osservazioni leopoldine [nt. 42], karton 23, p. 474

49 Rapporto di Kaunitz, 17 maggio 1780, in HHSAW, Vorträge, fz. 204. 
procedimento sommario, puniti "non già coll'inefficace solito precetto di emendarsi, e con una breve carcerazione, ma con pena corporale, cioè mediante l'inflizione di battiture come si usa in questi Stati, ed altrove" ${ }^{50}$ "La Casa di correzione doveva essere un castigo dell'ozio" e per gli autori "dei delitti meno gravi" ${ }^{51}$ al fine di "ridurli con tale castigo ad un più laborioso e migliore genere di vita". ${ }^{52}$ I dediti all'ozio, i piccoli borsaioli in tal modo sarebbero divenuti utili manifatturieri e avrebbero contratto "ivi l'abitudine di travagliare", come rilevava Firmian, nell'auspicare che il Senato si allontanasse dalla pratica di punirli con il carcere "dove a poco a poco divengono veri ladri". 53 Insomma, la Casa di correzione, come luogo di emenda per "discoli" e per i rei di lievi delitti, aveva trovato in queste convinzioni la sua origine. Degli anni Venti del Settecento era il progetto Trotti. Del 1749 è il progetto di Carlo Maria Recalcati, Capitano di giustizia, per il quale la Casa, destinata ad accogliere "mariti ubriachi e oziosi, teppistelli e giovinastri, donne lussuose e oziose, ruffiani e borsaruoli", avrebbe dovuto costituire una concreta alternativa al carcere, "scuola peggiore e nella quale non si lavora" ${ }^{54}$

Il primo concreto passo fu compiuto con l'istituzione, il 26 gennaio 1758, della Regia Delegazione sopra la Casa di correzione, dopo 4 anni iniziarono i lavori di edificazione che si protrassero per altri 4 anni. Causa della dilunga furono le difficoltà finanziarie. Nel settembre 1765 i lavori erano quasi ultimati e nel marzo dell'anno seguente vi fecero ingresso i primi detenuti. Di un progettato Albergo dei poveri, nulla si fece. A metà degli anni Ottanta, Giuseppe II avrebbe predisposto la costruzione di una Casa di lavoro libero da affiancarsi a quella di lavoro forzato. Sin dalla sua inaugurazione, e per molto tempo ancora, la Casa di correzione adempì una funzione diversa da quella originariamente divisata. Già dai primi mesi del '66 risultano rinchiusi 53 condannati alla galera che avrebbero dovuto espiare la pena in tutt'altro

50 Ibidem.

51 Rapporto di Kaunitz, maggio 1769, in HHSAW., Vorträge, fz. 202.

52 Rapporto di Kaunitz, 5 giugno 1775, in HHSAW., Vorträge, fz. 204

53 Il parere di Firmian si legge nel rapporto di Kaunitz, $1^{\circ}$ ottobre 1775, in HHSAW., Vorträge, fz. 204.

54 C.M. RECALCATI, consulta, 12 gennaio 1769, in Archivio di Stato Milano (ASM), Uffici giudiziari, p.a., cart. 207. 
luogo, fosse questo una nave straniera, il carcere pretorio o quello del Capitano di giustizia, o un apposito stabilimento come il futuro Ergastolo, e non certo un istituto destinato ad accogliere i corrigendi. Una commistione tra costoro e i delinquenti che si protrasse nel tempo, anche dopo l'istituzione dell'Ergastolo, e che tradiva la filosofia per la quale era stata pensata e voluta: quella della prevenzione e della riabilitazione, nonché della redenzione attraverso il lavoro e l'istruzione professionale. Insomma, la Casa di correzione funzionò quale valvola di sfogo per risolvere i problemi di sovraffollamento delle altre carceri. $\mathrm{Ma}$ non solo, in quanto v'erano motivi di carattere economico che determinavano forti resistenze al trasferimento all'Ergastolo dei galeotti e dei condannati al pubblico lavoro: si trattava, infatti, di evitare che i telai con tanto sforzo installati nella Casa rimanessero fermi per mancanza di mano d'opera.

Una svolta si ebbe con il dispaccio reale 16 ottobre 1775, che accoglie idee e progetti di Kaunitz: si attuava la direttiva di inviare i galeotti all'Ergastolo, considerandosi come male minore quello di mantenere i condannati al lavoro pubblico: ciò per non pregiudicare le manifatture e per farli comunque lavorare, anche se non pubblicamente, perdendosi, in tal modo, il fine dell'esemplarità della pena (Liva, 1990, pp. 70-75). A metà degli anni Ottanta si concretizza l'idea di realizzare una Casa di lavoro libero accanto a quella di lavoro forzato. Quest'ultima, i cui lavori ebbero inizio al principio del 1787, subito venne chiamata Casa di polizia. A mezzo 1788 poteva essere annunciato il completamento di questa fabbrica "aggiunta alla Casa di correzione ad uso di Casa di Polizia", ${ }^{55}$ ove, secondo il Piano legislativo redatto dal consigliere Secco e dal fiscale Risi - per la parte edile era stato interessato l'architetto Piermarini, ${ }^{56}$ - avrebbero dovuto trovar posto oziosi e mendicanti, i soli tenuti a lavorare, impiegati nella filatura della lana e del lino venendo remunerati con moneta della Casa stessa e, pertan-

55 ASM, Uffici giudiziari, p.a., cart. 258, 20 maggio 1788.

56 Si vedano le lettere di Wilczeck ai due commissari Secco e Risi, al Piermarini e al Taverna, assessore provinciale della Giunta delle pie fondazioni, dalla quale sarebbe dipesa la Casa di lavoro libero, in ASM, Uffici giudiziari, p.a., cart. 258. Il Piano è trasmesso a Kaunitz da Wilczeck con lettera accompagnatoria 22 agosto 1786 (ibidem); l'approvazione del Sovrano è comunicata con poscritto.alla lettera di Kaunitz a Wilczeck 2 ottobre 1786 (ibidem). 
to, solo in questa spendibile; i debitori, sino ad allora tenuti alla Malastalla, venivano trasferiti al nuovo reclusorio -proprio dall'alienazione dello stesso, unitamente a quella dell'Ergastolo che, "mediante il trasferimento dei condannati a Pizzighettone non è più d'alcun uso", era previsto che si ricavassero i fondi necessari per erigere il nuovo istituto- senza obbligo di lavoro, "giacché non venendo alimentati a spese della Casa, non debbono obbligarsi a lavorare per essa", godevano di un regime improntato ad una certa libertà; infine, gli arrestati segreti "rei di qualche delitto politico", sottoposti a regime segregante: occupavano stanze particolari, rimanendo sempre in isolamento, senza possibilità di parlare nemmeno con le guardie. Quanto alla Casa di lavoro libero, la sua sorte non fu di certo gloriosa: per la mancanza di fondi; per i locali che, destinati al lavoro, erano stati ricavati da quelli della stessa Casa di lavoro forzato, nonché per i lavori, che, di cattiva qualità, non erano né venduti né commissionati.

Il dibattito sull'erezione dell'Ergastolo si avvia negli anni Sessanta. La sua costituzione, anche se determinata in concreto dal rifiuto da parte di Venezia di accogliere i condannati al remo, fu, invero, il frutto di una libera scelta di politica criminale. Se è vero che, fino al 1764, il problema era stato quello di trovare per questi condannati un posto nelle sovraffollate carceri cittadine in attesa della loro effettiva traduzione al remo, è altresì vero che il problema cruciale risulterà di poi essere un altro. Lo pone in chiara evidenza il malcontento manifestato da Kaunitz - "Non so chi ne abbia avuto la commissione o l'incarico: so che siamo stati mal serviti, e lo cononoscerà anche l'E.V. [...]" ${ }^{17}$ - per l'operato del Capitano di giustizia Parravicini e del fiscale Besozzi, i quali, incaricati di elaborare un progetto "per rendere utili li condannati alla pena della galera", non avevano colto quale fosse il vero intento della sovrana: quello di far scontare la condanna ai propri sudditi in Patria, per non "perderli", certo, ma anche per il principio della esemplarità della pena, rimanendo pertanto insensibili alla ideologia ispiratrice, fondamentalmente utilitaristica, della politica che nel penale la Corte viennese intendeva perseguire. ${ }^{58}$ Il 18

57 Lettera di Kaunitz a Firmian, 2 giugno 1766, in ASM., Uffici giudiziari, p.a. cart. 207.

58 "Prego l'E.V. voler ritenere questo principio e far presente ancora alli Delegati per tal materia, che l'opera de' Sforzati cessa esser vantaggiosa quando non può effettuarsi senza spesa assai maggiore all'utilità cha allo Stato da quella risulta" (ibidem). 
luglio 1766 è al lavoro la Giunta, composta da Firmian, che la presiedeva, Della Silva, Santucci e Besozzi, la quale perviene a questa conclusione: avendo preso atto che si era "risolto che li sudditi di questo Regio Dominio, che non sono condannati in vita alla Galera, debbono impiegarsi al lavorerio delle fortificazioni”, stabiliva il principio per il quale al remo andavano inviati, oltre agli stranieri, i nazionali condannati a vita, $i$ protervi (vale a dire i recidivi), nonché, infine, i rei di atroci delitti. Tutti gli altri, invece, andavano internati nelle carceri, dalle quali, per fare posto, si traducevano alla Casa di correzione -"in cui si faranno esercire"- quei condannati che avessero posseduto "qualche arte, assegnando loro qualche mercede" ${ }^{59}$ Il problema si presentò, presumibilmente, nel 1772, quando si constatò l'eccessivo numero dei detenuti nella Casa di correzione e nell'Ergastolo, con pesante onere per l'Erario, poiché i Veneziani non accettavano più i nazionali condannati alla galera. ${ }^{60} \mathrm{Si}$ pensò di rivolgersi alla Spagna, ma invano. E dal 1781, i galeotti, tutti indistintamente, avrebbero scontato la pena all'Ergastolo. Se mai, il vero problema verteva sul come impiegarli.

Immergiamoci nella realtà dell'Ergastolo di Pizzighettone, quale risulta dalle carte beccariane. E facciamo subito conoscenza con Michele Donadeo, ispettore dell'Ergastolo, colui che soprintendeva alla vita che in esso, nei suoi molteplici aspetti, si svolgeva. Lo conosciamo nel 1789, in un voto steso da Beccaria e preceduto da una altisonante intitolazione: "Affare riservato e pervenuto al Dipartimento II senza numero, e riferito nel Regio Imperial Consiglio di Governo per ordine di Sua Eccellenza (i.e., Joseph Wilczeck) nella sessione 28 dicembre '89". Beccaria, "per obbedire ai venerati comandi di Sua Eccellenza", ha ripreso in esame la relazione inviatagli dall'Ispettore dell'Ergastolo, insieme con un Progetto di nuovo regolamento. "A tre punti principali si dirrigono le rappresentanze del Donadeo. Primo, propone secondo lui un nuovo regolamento dell'Ergastolo; secondo, chiede la rimozione de' due Assistenti e Coadiutore; terzo, nella rappresentanza a me diretta chiede, in vista delle circostanze che espone, un aumento di soldo". Per quanto riguarda il nuovo regolamento proposto, Beccaria non rile-

59 V. gli appuntamenti 18 luglio 1766, in ASM, Uffici giudiziari, p.a., cart. 207 [Liva, 1990, p. 108 s.].

60 Poscritto alla lettera di Kaunitz a Firmian, 27 agosto 1772, in ASM, Uffici giudiziari, p.a., c. 208. 
va sostanziali differenze con l'attuale, "supposto che questo sia tenuto in esatta e costante osservanza, di che vi è forte motivo di dubbitare, almeno per colpa de' subalterni, da quanto espone [...]" l'Ispettore stesso. ${ }^{61}$

Prima di esaminare le altre due "rappresentanze" di Michele Donadeo, mi pare utile passare in rassegna, in rapidissima rassegna $\mathrm{i}$ disordini che la non "esatta e costante osservanza" del regolamento comportavano. Veniamo a conoscenza di negligenze nella sorveglianza dei lavori, che si svolgevano nell'Ergastolo e di furti di materiali usati per tali lavori; ${ }^{62}$ di irregolarità commesse dall'Ispettore stesso e denunciate dal primo Assistente, il quale, dal canto suo, ha confessato di aver permesso che vari ergastolani andassero, sia pur sotto scorta, nei dintorni per visitare parenti, ciò su autorizzazione dell'Ispettore, secondo una prassi da tempo invalsa (Beccaria rileva: "[...] mi pare così incongruo e pericoloso quest' arbitrio che duro pena a credere che ciò possa mai essere stato autorizzato"); $;^{63}$ dell'uso da parte non solo dell'Ispettore, ma anche dei subalterni di far lavorare per proprio conto i condannati; ${ }^{64}$ irregolarità nella compilazione dei registri ${ }^{65}$ e dei "protocolli mensuali"; ${ }^{66}$ del fatto che era stato distribuito cuoio per far scarpe, ma parte di esso "erasi disperso", così come disperse risultavano non poche paia di scarpe; ${ }^{67}$ della fabbricazione di monete false in

61 BeCCarIa, Atti di Governo, XI [nt. 9], doc. 3473, 28 dicembre 1789, pp. 940-943.

62 C. Beccaria, Atti di Governo (serie VIII: 1790), a cura di R. CAnetTA, vol. XII dell'Edizione Nazionale delle Opere diretta da L. FIRPO e G. Francioni, Milano 2005 (d'ora in avanti, BeCCARIA, Atti di Governo, XII), doc. 3578, 15 febbraio 1790 , p. 165 .

63 Ibid., doc. 3609, 15 marzo 1790, p. 205 s.

64 BeCCARIA, Atti di Governo, XIII [nt. 46], doc. 3980, 26 marzo 1791, p. 78 s.

65 Ibidem.

66 BeCCARIA, Atti di Governo, XV [nt. 47], doc. 5698, 2 dicembre 1793, p. 709; doc. 5761, 30 dicembre 1793, p. 783, con riferimento all'"Ergastolo figliale di Mantova”; C. BECCARIA, Atti di Governo (serie XI: gennaio-dicembre 1794) a cura di R. CANETTA I (gennaio-agosto 1794), vol. XVI dell'Edizione Nazionale delle Opere diretta da L. Firpo e G. Francioni, Milano 2009 (d'ora innanzi, Beccaria, Atti di Governo, XVI-I), doc. 5873, 24 febbraio 1794, p. 148 s.

67 BeCCARIA, Atti di Governo, XII [nt. 62], doc. 3642, 19 aprile 1790, p. 251 s.: "Fu incaricata l'Intendenza Politica di Cremona di sentir quell'Ispettore e di metterlo in avvertenza perché in avvenire sia più oculato onde prevenire simili inconvenienti”. 
Ergastolo ${ }^{68}$ del prestito in danaro fatto da due condannati in favore del Coadiutore dell'Ergastolo; ${ }^{69}$ del mancato impiego della seta data all'assistente Cittelli per far corpetti ai condannati. ${ }^{70}$

Le accuse contro l'Ispettore andavano moltiplicandosi. Beccaria è prudente: si può pensare che tali accuse derivino da spirito di vendetta e non di verità. In ogni caso, sarebbe stato bene che l'Intendente politico - il marchese e patrizio lodigiano Matteo Sommaria [Mozzarelli, 1990, pp, 79, 83 s.] - assumesse "le più segrete ma accertate informazioni" e perfezionasse così in via economica le cose per farne relazione riservata al Consiglio di Governo, facendo però presente "all'Intendenza predetta che nelle informazioni stia ben lontano da qualunque forma di processo, al qual effetto potrà o personalmente portarsi nel luogo e sentire l'ispettore con la maggior moderazione, o eccitar riservatamente con lettera l'Ispettore medesimo a rispondere ai gravami $[\ldots]]^{71}$

Eguale prudenza Beccaria mostra di fronte al ragioniere dell'Ergastolo, Archinti, che chiede di poter giustificare le mancanze che gli vengono attribuite dal Donadeo. Egli accede all'intendimento negativo dell'Intendente politico: se ogni volta che un superiore riprende un inferiore si dovesse "instituire" una specie di processo, "come se si trattasse di punire un reo, di levargli l'impiego ecc., sarebbe troppo esposto il buon ordine e la disciplina, alla quale ogni impiegato è obbli-

68 Ibid., doc. 3738, 19 luglio 1790, p. 383. L'Ispettore dell'Ergastolo aveva altresì dato informazione della "morte di tre condannati mentre stavano eseguendo lavori nelle campagne".

69 BeCCARIA, Atti di Governo, XIII [nt. 46], doc. 4078, 6 giugno 1791, p. 193 s., ove si legge: "E' certamente poi più strano che chi veste, come il Maestri, la qualità d'impiegato nell'Ergastolo, e che deve essere sollecito di coadiuvare al buon ordine e al mantenimento d'una severa disciplina nel medesimo, ove qualunque benché minima indulgenza non può che rendere più audaci quei facinorosi ad abusarne, abbia avuta la poca prudenza o per meglio dire il disaprovevole coraggio di procurarsi in imprestito danaro dagli stessi condannati, e di rendersi per tal modo obbligato a' medesimi col nome in questo caso obrobriosissimo di debitore, e finalmente di esporsi, nelle premesse circostanze, a tutte quelle altre funeste conseguenze che e per la qualità del luogo e dei creditori con fondamento si possono temere". Il Maestri, pertanto, doveva essere punito: l'impunità l'avrebbe reso recidivo e avrebbe indotto altri impiegati a seguirne l'esempio.

70 BeCCARIA, Atti di Governo, XII [nt. 62], doc. 3705, 21 giugno 1790, p. 339.

71 Ibid., doc. 3532, 25 gennaio 1790, p. 106 s. 
gato". Importante, piuttosto, è il modo con il quale si fa la "riconvenzione", a voce o per iscritto, i toni più o meno severi. Se l'Intendente ha eseguito gli ordini del Consiglio nei termini esposti da Siro Archinti (cioè se i termini abbiano oltrepassato le prudenti misure con cui conviene fare simili "ripassate" ad un soggetto che non si vuol perdere, ma correggere) sarebbe stato meglio che egli avesse agito a voce. E poi, essendo emerso che il ragioniere si limitava, nell'esercizio delle sue funzioni, "alla pura e stretta scritturazione, che gli incombe come ragionato, che non invigilava, come è dovere, alla regolarità dei registri, forse distratto da altre occupazioni estranee all'Ergastolo, forse per i dissapori nati fra gli impiegati" (disfunzioni alle quali il Consiglio non avrebbe mancato di porre riparo) ha creduto di ammonirlo, perché raddoppi "quella attività e zelo che è dovuta da qualunque ha l'onore di trovarsi nel reale servizio". Insomma, l'Archinti poteva stare tranquillo o, meglio, raddoppiare i suoi sforzi sul lavoro: "la migliore giustificazione sarà quella di disimpegnare con zelo le proprie incombenze" ${ }^{72}$

Più complesso e delicato è il caso che Beccaria dovette affrontare con riguardo al canonico Gazzola, cappellano dell'Ergastolo. La sua vicenda nell'istituzione comincia bene, pur contrassegnata dalla proposizione di richieste. Richiedeva la qualifica di Parroco, un aumento del compenso di lire 300 assegnatogli per l'assistenza spirituale al carcere, assistenza molto gravosa che, per di più, gli aveva impedito di procurarsi una cura d'anime, con la relativa congrua. L'Intendente politico si mostra favorevole alla sua nomina quale coadiutore. Beccaria, dal canto suo, si rimette alla Commissione ecclesiastica "affinché si compiaccia di esaminare se vi sia luogo a consolare [che vi sia una vena d'ironia nella forma verbale usata?] il supplicante benemerito canonico Gazzola" ${ }^{73}$ Altre richieste: condono del "tenue" debito di lire 48.9 che egli aveva verso l'Ergastolo, concessione di impiegare quattro condannati in certi lavori riguardanti la riparazione della propria abitazione. Si tratta di richieste che Beccaria ritiene accoglibili, tenendo conto della "tenuità dell'oggetto" e del fatto che egli aveva disimpegnato "con lodevole zelo la laboriosa sua incombenza nel dirigere la coscienza dei condannati". Trattandosi di "concessione graziosa", Beccaria, comunque, interessa del caso la Conferenza Governativa, rimanendo in attesa "delle supe-

72 Ibid., doc. 3610, 15 marzo 1790, p. 207 s.

73 Ibid., doc. 3616, 29 marzo 1790, p. 215. 
riori determinazioni" ${ }^{74}$ "determinazioni", che sono favorevoli al cappellano: gli si condona il debito, gli si concede l'impiego gratuito di quattro condannati per due mesi per lo svolgimento di operazioni di riparazione alla casa domestica. ${ }^{75}$

Qualche nuvola comincia però ad addensarsi sul capo del "benemerito canonico" nell'aprile 1794. A protestare è Michele Donadeo, il quale ricorre contro Gazzola per alcune assenze, ma anche contro l'Intendenza provinciale delle finanze di Cremona, che aveva disposto su di un oggetto non rientrante nelle sue competenze. In particolare, l'Intendenza aveva inviato un sacerdote che coadiuvasse il Gazzola nel compimento degli esercizi spirituali senza prima accertarsi che il sacerdote fosse beneviso all'Ispettore, che, come si legge nel voto di Beccaria, è "caricato della responsabilità in tutti gli oggetti che riguardano quel luogo di pena"; tanto più poi ha mancato, in quanto che la Regia Intendenza non può ignorare i disapori che passano tra detto Sacerdote e l'Ispettore". Ma ha mancato anche "il Canonico Gazzola col cercare detto Sacerdote, appunto perché esso pure non può ignorare i mentovati dissapori". Ecco quindi l'nvito: che in futuro "si astenga di chiedere in aiuto sacerdoti che non siano benevisi all'Ispettore $[\ldots]$ ”. ${ }^{76}$

A maggio 1794 si viene a conoscenza che il Gazzola esige dalla cassa dell'Ergastolo "la mercede della messa, applicando la messa medesima ad altri, per percepire così un'altra mercede”. E Beccaria scrive: "Non può poi il Relatore omettere di far presente la necessità di far che le messe siano celebrate nell'Ergastolo in tutti i giorni -Gazzola chiederà che venga tolto l'avviso dell'Ispettore che proibiva la celebrazione della messa quotidiana, secondo un'accusa destinata a cadere, ${ }^{77}$ a condizione però che le medesime sieno applicate per Sua Maestà, da cui viene corrisposta al Sacerdote la mercede". ${ }^{78}$ Beccaria chiede poi informazioni al Vescovo di Cremona, al fine di acclarare "quale sia la condotta e quali i costumi del canonico Giovanni Maria Gazzola". Le

74 BeCCARIA, Atti di Governo, XIV [nt. 47], doc. 4768, 16 luglio 1792, p. 328 s.

75 Ibid., doc. 4813, 6 agosto 1792, p. 385. Occorreva comunque prestare grande attenzione perché dalla concessione di servirsi dell'opera dei condannati "non nasca abuso od inconveniente". Si veda anche doc. 4865, 3 settembre 1792, p. 450.

76 BeCCARIA, Atti di Governo, XVI-I [nt. 66], doc. 5995, 22 aprile 1794, p. 303 s.

77 Ibid., doc. 6098, 2 giugno 1794, p. 437.

78 Ibid., doc. 6052, 19 maggio 1794, p. 384. 
informazioni, secondo l'ormai nota prudenza di Beccaria, avrebbero dovuto essere riservate, "prescindendo da qualunque apparato giudiziario" ${ }^{79}$ La promessa del Vescovo di ammonire il cappellano non è sufficiente, anche perché i riscontri della Curia vescovile non gli sono per nulla favorevoli. Beccaria è dell'intendimento di non "continuarlo nel geloso impiego che copre", perché in un luogo di pena "anche le picciole mancanze si devono guardare come gravi”. Crede pertanto di far consulta alla Reale Conferenza per rimuovere questo soggetto, "per destinarne un altro che sia dotato di sufficiente sapere e di prudenza" e che sia in grado, cosa importante, "di sodisfare anche all'Ispettore dell'Ergastolo, responsale degli inconvenienti che possono nascere in quel luogo di pena" ${ }^{80}$ Il 15 ottobre Beccaria informava il Vescovo di Cremona che il Magistrato Politico Camerale aveva deciso di sostituire il Gazzola con un altro sacerdote e, al contempo, manifestava il suo sempre vivo sentimento di umanità: "Per i giusti riguardi, però, che si devono ad un sacerdote che copre un canonicato nel suddetto borgo", prima di rimuoverlo formalmente dalla predetta incombenza, "gli ha fatto riservatamente insinuare di rinunciarla, su di che si attendono riscontri". ${ }^{81} \mathrm{Il}$ riscontro arrivò ben presto: due settimane dopo Beccaria informava l'Ispettore Donadeo che il Canonico Gazzola aveva "implorato dal Magistrato Politico Camerale il permesso di rinunciare alla di lui incumbenza di Cappellano dell'Ergastolo [...]". ${ }^{82}$

Se intenso e vivo era in Beccaria penalista e pubblico amministratore lo spirito umanitario, non meno vivo era il severo rigore al quale lo richiamava la ragione in nome dei principi di legalità e di certezza del diritto. Alessandro Manzoni aveva scorto in Beccaria colui che aveva riunito "la commozione e il raziocinio", che aveva affrettato "il trionfo della ragione e della umanità [...]" gersi della vita nell'Ergastolo di Pizzighettone pone talora Beccaria di fronte a vicende in cui, sia pur nel piccolo, umanità e ragione sembra-

79 Ibid., doc. 6157, 23 giugno 1794, p. 506 s.

80 Ibid., doc. 6221, 28 luglio 1794, p. 575.

81 BeCCARIA, Atti di Governo, XVI-II [nt. 18], doc. 6384, 15 settembre 1794, p. $764 \mathrm{~s}$.

82 Ibid., doc. 6431, 29 settembre 1794, p. 821 s.

83 A. MAnzoni, Appendice storica su la colonna infame (primo abbozzo del 1823), in Tutte le opere di Alessandro Manzoni, a cura di A. CHIARI e F. GHISALBERTI, volume secondo tomo terzo, Milano 1968, p. 683. 
no confliggere. Un detenuto era fuggito dall'Ergastolo, filiale di Mantova: il custode dei condannati e quattro guardie erano state arrestate e sottoposte a processo. Per quanto riguarda il custode, Desiderio Ferrari, il Delegato assessore Guerrieri aveva deciso che, durante lo svolgimento del processo, alla sua famiglia venisse corrisposto la metà del salario. Beccaria non ritiene corretta siffatta decisione, però riflette che la famiglia del Ferrari era stata di recente trasferita da Pizzighettone a Mantova e per questo "trovandosi in un paese nuovo, sarebbe sforzata a mendicar per vivere". Questa considerazione lo induce a non opporsi al provvedimento, che pur non era conforme alla legge, ma a rimettere al Magistrato politico camerale la decisione se sia o no conveniente "approvare la determinazione di quel delegato fin a processo finito". Una minuta di lettera del 26 agosto 1793 c'informa che al Magistrato era pervenuta la sentenza pronunciata dal Tribunale d'appello di Mantova, che aveva riconosciuto colpevole il Ferrari e non ree le quattro guardie. Ad esse, pertanto, doveva essere corrisposto il salario dovuto, mentre "detto Vice Capo [Ferrari] resta per sei mesi sospeso dal suo esercizio, e perciò non dovrà corrispondersegli alcun soldo, su di che ha il Magistrato medesimo date le opportune disposizioni alla regia Intendenza di Finanza in Cremona, nell'incontro che ha alla medesima ordinato di far cessare la corresponsione della metà del soldo di detto Ferrari che, durante il di lui arresto, venne dal Dicastero accordata alla di lui famiglia". ${ }^{84}$

Più interessante e complesso è il caso che, estraneo alla realtà penitenziaria lombarda, vide coinvolto il perito camerale Giuseppe Gaeta, ingegnere presso la Regia Amministrazione, un caso che ha già attratto l'interesse della storiografia (Canetta, 1973, pp. 34, 82 s.; 2009, p. 1062).

84 BeCCARIA, Atti di Governo, XV [nt. 47], doc. 5373, 17 giugno 1793, p. 349 doc. 5389, 24 giugno 1793, p. 365; doc. 5501, 26 agosto 1793, p. 490 s. Posso ricordare, allontanandomi da Pizzighettone, il caso di tal Lorenzo Odelli condannato, come introduttore di sale estero, a due anni di Casa di correzione ed alla confisca dei beni. Beccaria accede al voto del Fiscale, per il quale, in contrasto con la rigorosa applicazione della legge, l'oggetto della confisca, poche e misere suppellettili, doveva essere restituito alla moglie del contrabbandiere. Di fatto, intervenne successivamente la concessione della grazia da parte dell'Arciduca Ferdinando, con il conseguente provvedimento che concedeva all'interessata il ritiro dei beni sequestrati (BECCARIA, Atti di Governo, XVI-II [nt. 18], Addenda doc. 632.1, 6 e 10 febbraio 1781, p. 1013; doc. 638.1, 10 marzo 1781, p. 1014; doc. 645.1, 2 e 4 aprile 1781, p. 1015. 
Costui si era reso colpevole di comportamenti scorretti ed arbitrari -sostanzialmente consistenti nel taglio e vendita di piante di pertinenza della vacante Abbazia di Santo Spirito di Terzago senza intervento e approvazione della Regia Amministrazione del fondo di Religione in Milano-, che avevano indotto il Procuratore generale a richiederne la rimozione dall'impiego. Una richiesta ispirata a rigore, dunque, che sembra trovare consenziente Beccaria: un'Amministrazione che negli affari più delicati e rilevanti si affidi ai periti deve riporre in costoro la massima fiducia, una fiducia che però deve essere meritata. La loro condotta deve essere "esente non solo da ogni macchia, ma anche da ogni benché menomo sospetto". E Giuseppe Gaeta, questa fiducia, l'aveva tradita. Ma le considerazioni di Beccaria non si fermano qui: l'Ingegnere ha già sofferto la sospensione dell'incarico per il tempo di sei mesi; è, questa, la prima mancanza nella quale sia incorso ed è, pertanto, fondata la speranza che essa rimanga anche l'unica, dal momento che "[...] ove si tratti di un unico atto, si rende più probabile l'emenda". E ancora, Beccaria rivela la sua sensibilità ai diversi riflessi che una mancanza ha nel privato e nel pubblico: la condotta riprovevole attuata nel privato comporta la perdita di una parte soltanto della clientela, mentre nel pubblico "se [...] vien dimesso dall'ufficio dell'Amministrazione, la cosa non può più stare occulta, s'ingrandisce, come per l'ordinario accade, il titolo della dimissione e, col perdere l'impiego, l'Ingegnere Gaeta viene certamente a perdere tutte le clientele, e destituito quindi assolutamente di ogni mezzo per sussistere una intiera famiglia". ${ }^{5}$ Un mese dopo, siamo nel settembre del 1794, quasi al limitar della vita di Cesare Beccaria, una sua consulta c'informa che il Procuratore generale, pur rimanendo fermo nel suo convincimento circa l'opportunità della rimozione dall'impiego dell'Ingegnere, proponeva "che si potesse portare l'affare alla cognizione della Reale Conferenza Governativa, accennando che talvolta i Dicasteri superiori possono trovare delle plausibili considerazioni per non attenersi al rigore di ragione, da cui, per proprio istituto, ritenne il Procuratore Generale di non potersi dipartire [...]".

$\mathrm{Ci}$ ritroviamo così posti, al vivo, di fronte al binomio rigore di ragione, incarnato, nel caso, dal Procuratore generale, e spirito di umanità, del quale si fa alfiere Cesare Beccaria. "[...] Non possiamo [...]

85 BeCCARIA, Atti di Governo, XVI-I [nt. 66], doc. 6321, 25 agosto 1794, pp. 688-690. 
dissimulare che siffatta pena [le dimissioni] ci sembra troppo rigorosa in confronto delle circostanze concomitanti la mancanza di cui il detto Ingegnere viene imputato": egli neppur ha tentato di appropriarsi del ricavato della vendita delle piante; non ha preteso "una partecipazione del prezzo medesimo, oppure chiesta una somma coll'offerta di qualche facilitazione nella perizia", della quale era stato richiesto; manco ricorre il sospetto di dolo nei suoi comportamenti, che non paiono, pertanto meritevoli della "grave infamante pena della cassazione del Gaeta dall'ufficio d'ingegnere", una pena che "lo renderebbe per così dire civilmente morto rapporto al di lui impiego [...]".

Quale sanzione risulterebbe allora, secondo Beccaria, proporzionata alla natura del caso? "Noi saremmo del subordinato sentimento" che, tenendo conto della già sofferta sospensione dal servizio, il Gaeta possa essere ripristinato nell'impiego, previa ammonizione a non tener più in avvenire consimili comportamenti, sotto pena di essere, in caso di inosservanza del precetto, "rimosso senza speranza di esser più repristinato". E poiché durante la sospensione dal servizio, egli ha continuato a "percepire il soldo", di tale soldo dovrà restare privo per il tempo che piacerà alla Reale Conferenza Governativa, oltre a dover reintegrare il fondo di Religione degli eventuali danni sofferti. La consulta si conclude con queste parole: "Per tale maniera, mitigando il rigore fiscale, noi crederessimo bastantemente punito l'Ingegnere Gaeta delle sue irregolarità e mancanze, e siccome trattasi di un impiegato stato sospeso e di cui è stata progettata la rimozione dal Regio Fisco, ci siamo trovati in dovere di subordinare l'emergente alla superiore cognizione della Reale Conferenza Governativa, stando nella rispettosa attenzione di quelle determinazioni che vorrà degnarsi di prendere". ${ }^{86}$ Si tratta di una vicenda sulla quale mi sono soffermato, perché pone in risalto alcuni dei caratteri più significativi della personalità scientifica e umana di Beccaria penalista, di un Beccaria che si avvale di quelle "plausibili -plausibili, perché non tradiscono il principio di legalità- considerazioni per non attenersi al rigore di ragione", alle quali faceva riferimento il Procuratore generale: principio della proporzionalità e della umanità della pena, fiducia nella emenda del reo.

86 BeCCARIA, Atti di Governo, XVI-II [nt. 18], doc. 6435, 29 settembre 1794, pp. 826-832. 
Ma ritorniamo ai disordini che si verificavano nell'Ergastolo di Pizzighettone: un altro riguardava lo spaccio di vino a guardie e condannati. Lo si viene a conoscere quando il Capo guardia dell'Ergastolo chiede di non pagare più la tassa di 45 lire per ogni brenta di vino che dà, per l'appunto, a guardie ed a carcerati. Beccaria vuol saperne di più, prima che il Consiglio di Governo decida in merito: quando ha avuto origine il pagamento di questa tassa? Su che è fondata? A vantaggio di chi va, della Finanza o della cassa dell'Ergastolo ${ }^{87}$ La tassa era stata introdotta il $1^{\circ}$ maggio 1786 dall'Ispettore Redaelli ed il suo ammontare andava a vantaggio dell'istituto di pena. ${ }^{88}$ Non mi soffermo sui vari interventi dei diversi organi e autorità che discussero sulla opportunità della vendita di vino all'interno di un istituto di pena, qual era l'Ergastolo, sulla determinazione di un equo prezzo di siffatta vendita. Il 10 maggio 1790 Beccaria pone fine alla faccenda. L'Ispettore era dell'idea che fosse opportuna la proibizione della vendita; l'Intendente politico riteneva invece utile che i condannati potessero godere dell'uso del vino, utile a preservarli da molte malattie, massime lo scorbuto, ponendo in rilievo che la bevanda potesse essere venduta a titolo di grazia o di premio a coloro che dessero segni di ravvedimento o che avessero problemi di salute. E Beccaria? Liberato il capo custode dal peso arbitrario del pagamento delle 45 lire per brenta di vino a vantaggio dell'Ergastolo, era ammissibile la vendita del vino al minuto a guardie ed a condannati, vendita che doveva avvenire al prezzo che non oltrepassasse le 4 lire di utile sul prezzo della compera, vendita che doveva inoltre essere giustificata di volta in volta dall'Ispettore. Ciò sotto pena della sospensione ed anche maggiore in caso di abuso. In linea di massima era proibito ai condannati di comprar vino (per le guardie Beccaria si rimetteva al Consiglio, il quale avrebbe dovuto valutare che cosa fosse preferibile: che bevessero il vino in loco ovvero nelle bettole?), salvo per quelli in ordine ai quali l'Ispettore, usando di prudente arbitrio, credesse di permetterlo, o per animarli, in occorrenza di opere di maggior fatica, premiandoli con tale condiscendenza, o come più morigerati degli altri, o come utile rimedio preventivo contro lo scorbuto. "Un tal mezzo, bene adoperato, può molto giovare alla disciplina di quel luogo e alla conservazione

87 BeCCARIA, Atti di Governo, XI [nt. 9], doc. 3414, 9 dicembre 1789, p. 869.

88 Ibid., doc. 3470, 28 dicembre 1789, p. 937. 
di quel peculio che è troppo importante che sia riservato alla fine di pena per i condannati ad tempus". ${ }^{89}$

Un aspetto, questo della sorte, della vita stessa dei condannati una volta ritornati in società, al quale Beccaria si mostra particolarmente sensibile, come chiaramente rivelano le considerazioni svolte intorno a quelle "rappresentanze", che ci hanno permesso di conoscere l'ispettore dell'Ergastolo di Pizzighettone, Michele Donadeo. Della prima "rappresentanza”, della quale già si è fatto cenno e consistente nel progetto di un nuovo regolamento, due punti attrassero l'attenzione di Beccaria. Mentre il $\mathbb{S}$ Occorrendogli di far fare induceva la presunzione che l'Ispettore potesse ordinare manifatture ai condannati per proprio conto, il che era contrario a quanto disposto in precedenza dalla Camera dei Conti e dal Consiglio di Governo, il $\mathbb{S}$ Non essendo poi lasciava intendere che l'Assistente ai lavori avesse la facoltà di far percepire ai condannati il terzo o la metà delle loro fatture: "dove si vuol dare al principiante qualche miglior tratamento per animarlo al lavoro per poi toglierlo al ammaestrato, ciocché non sembra conforme al indole rigorosa di un luogo di pena, e più addattato ad una casa di correzione che ad un ergastolo". Se l'intento è invece quello di mettere a disposizione qualche tenue somma all'Assistente da distribuire ai condannati come remunerazione delle loro fatture, ed allora occorre la massima prudenza al fine di evitare che i condannati ad tempus, che sono il maggior numero, al momento della loro liberazione si ritrovino senza denaro. Occorre piuttosto far sì che sia loro "riservato il maggior peculio possibile affinché, fatti liberi, non si trovino quasi in necessità di ritornare ai delitti”. La sorte dei condannati, una volta scontata la pena, preoccupava anche la Corte. Come potranno gli ex-ergastolani "senza mezzi di sostentamento iniziare una vita migliore di quella passata [...]"? Il II Dipartimento, nella persona di Cesare Beccaria, "nel convenire colla superiore osservazione", configurava una duplice soluzione, ribadendo, in buona sostanza, il pensiero ora ricordato: si sarebbe potuto o dovuto moltiplicare i lavori ovvero, attraverso una più severa disciplina, diminuire le occasioni di spesa "per serbar loro un peculio al termine della pena [...]". ${ }^{90}$

Anche su di altri punti del Regolamento proposto si sofferma

89 BeCCARIA, Atti di Governo, XII [nt. 62], doc. 3668, 10 maggio 1790, p. 285 s.

90 BeCCARIA, Atti di Governo, XI [nt. 9], doc. 3473, 28 dicembre 1789, p. 941. s.; XII [nt. 62], doc. 3658, 3 maggio 1790, p. 270 s. (osservazioni e riscontri al n.ro 423). 
Beccaria: in esso si dava facoltà alle guardie di "scaricare l'archibugio" contro chi si allontanasse dal lavoro; si prevedeva l'irrogazione di castighi e di multe ai condannati. Era materia assai delicata, che non poteva essere lasciata indefinita, occorreva, piuttosto, precisione, la massima precisione, anche perché la loro inflizione si rendeva effettivamente necessaria in un Ergastolo. Per la loro determinazione e applicazione occorreva concertarsi con il Supremo Tribunale di giustizia che, del resto, per mezzo della Commissione mista, aveva già determinato i tipi del castigo, che l'Ispettore poteva applicare rispetto alle piccole delinquenze dei condannati. In merito, un anno dopo, il Supremo Tribunale di giustizia riferiva al II Dipartimento del Consiglio del Governo di una nota ricevuta dal Tribunale d'appello relativa ad un appuntamento della Commissione giudiziario-politica circa la qualità dei delitti e delle pene da lasciare in facoltà (il che vuol dire all'arbitrio) dell'Ispettore dell'Ergastolo. Ciò preoccupava l'alta magistratura, perché poteva risultare alterato il disposto delle norme del 1788, che affidavano la vigilanza sul trattamento e sulla disciplina dei condannati all'Ergastolo, per l'appunto, al Tribunale d'appello.

Proprio al Tribunale d'appello era stato richiesto dal Supremo Tribunale di giustizia un parere su un Piano proposto dall'Intendenza politica di Cremona relativo agli aguzzini dell'Ergastolo di Pizzighettone: trasmesso poi alla Commissione giudiziario-politica, "eccitata la Corte de' Conti", il parere sarebbe pervenuto, da ultimo, al Consiglio di governo. ${ }^{91}$ E Beccaria fece sentire la sua voce. In contrasto con quanto opinato dalla Camera dei Conti, Beccaria non vedeva la necessità che il capo aguzzino e i sottoaguzzini, in numero di due o tre, fossero scelti tra i liberi (l'Intendenza politica riteneva che tra costoro dovesse essere scelto solo il capo). Il motivo? " $[\ldots]$ non sarà così facile di trovar fra persone mediocremente oneste chi si adatti a questo libero esercizio, massimamente che la paga conveniente non potrebbe che riuscire di qualche sensibile aggravio alla Camera". E, pertanto: capo, da scegliersi tra i libe-

91 BeCCARIA, Atti di Governo, XI [nt. 9], doc. 3473, 28 dicembre 1789, p. 940943, in particolare, p. 942; XII [nt. 62], doc. 3578, 15 febbraio 1790, p. 165; doc. 3899 , 15 novembre 1790, p. 616. Sugli interventi dei diversi Organi di giustizia, BECCARIA, Atti di Governo, XI [nt. 9], doc. 3395, 30 novembre 1789, p. 842; XII [nt. 62], doc. 3654, 3 maggio 1790, p. 266; doc. 3677, 17 maggio 1790, p. 300. 
ri, coadiuvato da due aiutanti "fra i condannati, da sciegliersi fra i meno cattivi e meno rei di loro". Al capo 20 soldi al giorno; ai condannati 5 soldi al giorno. Secondo Beccaria, l'aumento, invero tenue, rispetto alla paga corrente, 15 e 4 soldi, rispettivamente, non costituirebbe un aggravio sensibile (lire 182.10 in più all'anno) e, d'altra parte, "è più reperibile un agozzino con quella paga che coll'altra troppo tenue, e i condannati sotto agozzini saranno più animati all'adempimento del loro dovere, massime che questi potrebbero essere amovibili ad nutum dell'Ispettore". ${ }^{2}$

Le ragioni della sicurezza sarebbero prevalse sulle ragioni dell'economia ed anche la paga sarebbe aumentata nella sua entità. Nel marzo 1791 l'Ispettore dell'Ergastolo doveva procedere per "invenire gli agozzini liberi" con la previsione di una "mercede di soldi 25 al giorno". E la scelta doveva cadere su persone dai favorevoli "riscontri". Sull'utilità e fondatezza di tali "riscontri" è possibile avanzare, per lo meno, qualche perplessità. Nel luglio 1791 il Capitano di giustizia inviava informazioni intorno a due persone che avevano richiesto di essere assunte come aguzzini nell'Ergastolo: si trattava di tal Ambrogio Cerutti, milanese, che chiedeva 35 soldi giornalieri, e di tal Giacomo Iulitta, novarese, che, di soldi, ne chiedeva 25. Secondo Beccaria, Iulitta poteva "portarsi tosto all'Ergastolo di Pizzighettone ad esercitare l'incumbenza di agozino". Quanto al Cerutti, lo si doveva convocare per comunicargli che il Magistrato non poteva accordargli quanto richiesto, ma "soldi ventinove danari nove, giusta quanto si praticherà col Iulitta". Il soldo degli aguzzini era stato fissato nella misura indicata "all'effetto di distinguerli, in qualche parte, dalle guardie, alle quali restano assegnati soldi 30 , e ciò per ovviare il malcontento di queste nel vedere ad esse parificato l'agozzino, malcontento che al dire di quell'Ispettore si è già manifestato".

L'anno seguente, il Capitano di giustizia proponeva un aumento di soldi 2 denari 9 rispetto alla mercede corrente ch'era di soldi 29.9 a tal Gajani, che avrebbe dovuto sostituire Giacomo Reda "interinalmente accettato per secondo agozino nell'ergastolo di Pizzighettone", il quale era risultato "reo di delitti nello Stato sardo, ed anzi condannato in contumacia a due anni di galera [...]". Costui, allontanatosi improvvisamente dalla sede, sarebbe stato sostituito da un altro aguz-

92 Ibid., doc. 3900, 15 novembre 1790, pp. 617-619. 
zino, i cui "riscontri", che non conosciamo, saranno stati sperabilmente migliori di quelli di Giacomo Reda."

Alla Corte, che avanzava dubbi circa l'utilità della presenza di un camparo presso l'Ergastolo, non riuscendo a immaginare in quali mansioni avrebbe potuto essere utilmente impiegato, Beccaria faceva rilevare che il compito consisteva "nell'invigilare sulla condotta delle guardie che custodiscono i condannati fuori dell'Ergastolo", sui lavori svolti dai condannati e, soprattutto, nella custodia del luogo ove sono riposti gli attrezzi utili a tali lavori. Del resto, "sull'utilità di detto camparo ha avuto il Consiglio dell'Intendenza politica di Cremona, anche recentemente, assentati riscontri" ${ }^{94}$ In ogni caso, tutti costoro, per quanto costretti all'interno dell'Ergastolo, avrebbero dovuto tenere i ceppi ai piedi; la scelta delle persone era affidata all'Ispettore, ma la nomina era subordinata all'approvazione del Tribunale d'appello, "colla specificazione dei titoli, qualità e tempo della condanna [...]"; era in potere di tali guardie di percuotere gli ergastolani, "non a colpi di palosso, che si crede pericoloso come lo credo anch'io [...], ma a colpi di nervate, limitandoli però a qualche percossa, e restringendo tale facoltà delle guardie a' limiti di un semplice ricordo al condannato per indurlo a fare il proprio dovere". ${ }^{5}$ Non solo con il nerbo o con il bastone i condannati potevano essere eccitati al lavoro o ricondotti alla disciplina, ma anche con le multe. In ordine ad esse, ritroviamo qui il Beccaria dei Delitti e delle pene così come

93 BeCCARIA, Atti di Governo, XIII [nt. 46], doc. 3974, 14 marzo 1791, p. 70; doc. 4117, 4 luglio 1791, p. 243. BeCCARIA, Atti di Governo, XIV [nt. 47], doc. 4712, 11 giugno 1792 , p. 263; doc. 4714, 11 giugno 1792, p. 265; doc. 4715, 11 giugno 1792, p. 266; doc. 4727, 18 giugno 1792, p. 280.

94 BeCCARIA, Atti di Governo, XII [nt. 62], doc. 3658, 3 maggio 1790, p. 273 (osservazioni e riscontri al n.ro 692).

95 Ibid., doc. 3900, 15 novembre 1790, pp. 617-619. In questa materia, pertanto, è possibile cogliere quelle note di prudenza e di equità che mi pare caratterizzino la personalità di Beccaria, anche nelle vesti di alto funzionario di Stato. Egli riteneva che il Consiglio di Governo potesse convenire sull'opportunità che, in caso di esecuzione di opere esterne all'Ergastolo eseguite dai condannati, venissero predisposte, con funzioni di sorveglianza, una o due guardie, a seconda del numero degli ergastolani impegnati, ricompensate con 10 soldi in più al giorno per questa ulteriore incombenza. Ebbene: "[...] siccome tale incumbenza è sotto un aspetto lucrosa e sotto l'altro odiosa, così crederei che tale peso o ufficio dovesse esercitarsi per un turno da fissarsi dall'Ispettore, togliendo così al medesimo quella taccia di parzialità che conviene per quanto si può prevenire". 
l'affermazione del principio illuministico della certezza del diritto, della legalità della pena. Ebbene, "con riguardo alle multe siami permesso di osservare -scrive Beccaria- che non mi sembrano le più adattate alla situazione di un condannato, al quale più che senso fa torto la mutilazione del peculio da serbarsegli al finire della pena" ${ }^{96}$ Parole in cui risuona l'eco del principio della proporzionalità della pena.

Rimarrebbe da dire delle due altre rappresentanze avanzate da Michele Donadeo -si è in precedenza esaminata la prima-, nel momento in cui abbiamo fatto la sua conoscenza. Ebbene, con la seconda, l'Ispettore rivelava il suo reciso scontento nei confronti dei due Assistenti e del Coadiutore, tanto reciso e netto che di costoro intendeva disfarsi. Beccaria invita, ancora una volta, alla prudenza. Rimuovere tre soggetti ad un colpo, senza aver sotto mano i sostituti "era un azardare di troppo l'economia di quel luogo". E poiché il più aggravato era uno dei due Assistenti, il Cittelli, si poteva, se mai, cominciare da lui, nel dare un taglio. Il che avrebbe potuto sollecitare gli altri due indiziati di "taglio" ad adempire con maggior correttezza e solerzia il loro dovere.

Con la terza rappresentanza, Michele Donadeo chiedeva un aumento di soldo di 24.000 lire: Beccaria non è per nulla favorevole a siffatta richiesta, anche perché, meglio, soprattutto perché la Camera dei Conti aveva pensato piuttosto che "a misura dai vantaggi che potrà ricavare il luogo della buona economia dell'Ergastolo e dei vantaggi che possono derivare dai lavori dei condannati" l'Ispettore avrebbe potuto essere annualmente gratificato. Beccaria è sorpreso, piuttosto, dalla tenue mercede corrisposta allo scrittore dell'Ergastolo, Giovanni Battista Donadeo, fratello minore dell'Ispettore. Le 300 lire contemplate non permettevano ad un impiegato, che deve quotidianamente aiutare l'Ispettore nelle moltissime scritture che debbano essere fatte, di condurre una vita, che fosse almeno sostenibile. In ogni caso era comunque opportuno sentire la Camera dei Conti, pur ribadendo la necessità dell'aumento di mercede, anche perché, "essendo fratello dell'Ispettore non dovrebbe sperare nell'Ergastolo un alto più utile impiego $[\ldots] " .{ }^{97}$

Qualche avanzamento contraddistinguerà invero la carriera di G.B. Donadeo, anche se presso la filiale (sede distaccata) dell'Ergastolo

96 BeCCARIA, Atti di Governo, XI [nt. 9], doc. 3473, 28 dicembre 1789, p. 942.

97 Ibidem. 
di Mantova. Seguirò schematicamente la vicenda che lo riguarda anche perché veniamo a conoscenza della complessità e, pertanto, della conseguente inevitabile lunghezza dell'iter procedurale di una vicenda che, di per sé, certamente complicata non è.

4 ottobre 1790: al II Dipartimento perviene, per il tramite dell'Intendenza Politica di Cremona la supplica con la quale Giovan Battista Donadeo, scrittore, richiede un aumento dell'attuale salario, quelle 300 lire che anche Beccaria aveva ritenuto, nella sua modestia, ai limiti della sopravvivenza, aumento che non comprende la sua ulteriore mansione di direttore interinale dei lavori dei condannati a Mantova. Chiede anche una gratifica per aver introdotto diverse manifatture nell'Ergastolo. ${ }^{98}$

8 Agosto 1791: il Donadeo rinnova la richiesta. Beccaria sollecita l'Intendenza Provinciale della Finanza di Cremona perché si pronunci se e in quale misura "sia praticabile l'esposto". 99

3 Ottobre 1791: il Donadeo, direttore interinale dei lavori a Mantova, chiede di essere assunto in pianta stabile. Anche in questo caso Beccaria richiede l'intervento dell'Intendenza Provinciale delle Finanze di Cremona. ${ }^{100}$

17 Ottobre 1791: perviene a Beccaria la notizia che l'Intendente delle Finanze di Cremona si recherà a Pizzighettone per decidere l'entità della gratifica da corrispondere al Donadeo. Di tale visita, dice Beccaria, si attende l'esito perché il Magistrato Camerale possa dare "evasione al ricorso". ${ }^{101}$

30 gennaio 1792: l'Intendente Provinciale di Cremona restituisce con il proprio parere le carte che corredano la richiesta di gratificazione del Donadeo: Beccaria le trasmette alla Camere de' Conti. ${ }^{102}$

5 Marzo 1792: la Conferenza Governativa trasmette la richiesta del Donadeo di essere remunerato come direttore in pianta stabile dell'Ergastolo, sede di Mantova. E' richiesto il parere del Magistrato Politico Camerale. ${ }^{103}$

\footnotetext{
98 BeCCARIA, Atti di Governo, XII [nt. 62], doc. 3852, 4 ottobre 1790, p. 557.

99 BeCCARIA, Atti di Governo, XIII [nt. 46], doc. 4203, 8 agosto 1791, p. 352

100 Ibid., doc. 4354, 3 ottobre 1791, p. 536.

101 Ibid., doc. 4377, 17 ottobre 1791, p. 566.

102 BeCCARIA, Atti di Governo, XIV [nt. 47], doc. 4522, 30 gennaio 1792, p. 49.

103 Ibid., doc. 4565, 5 marzo 1792, p. 98.
} 
5 Marzo 1792: il Donadeo espone le ragioni per le quali ritiene di meritare un salario fisso, esibendo anche il prospetto degli introiti dovuti alle manifatture eseguite dai condannati dal 1788 al $1792 .{ }^{104}$

21 Maggio 1792: l'Intendenza Provinciale delle Finanze di Cremona chiede il rimborso spese sostenute dall'Ispettore Barbò in occasione della visita all'Ergastolo. ${ }^{105}$

2 Dicembre 1793: l'Intendenza Provinciale delle Finanze invia, con il proprio parere, la richiesta del Donadeo, II Assistente presso l'Ergastolo, filiale di Mantova, di godere di una gratificazione anche per alleggerirsi del debito di L. 1.537.6.8 che ha verso l'Ergastolo di Pizzighettone. ${ }^{106}$

179424 febbraio: l'Assistente Silvio Manini si lamenta che in Mantova deve svolgere mansioni che non gli competono. Fa presente che vive fuori città con maggiorazione di spese. Beccaria rileva che il Manini ha il compito di redigere i "protocolli mensuali". Rivelatosi incapace, la sua funzione è stata trasferita al II Assistente, il Donadeo. In buona sostanza, il Manini ha meno da fare ed a Beccaria non pare conveniente l'attribuzione di gratificazione alcuna, ovvero di un aumento di salario. Però, l'aumento sensibile di spesa, dovuto al fatto che vive fuori casa, dovrebbe comportare il riconoscimento di un monte spese per l'affitto della casa di L. $100{ }^{107}$

16 Giugno 1794: il Donadeo, Assistente presso l'Ergastolo, filiale di Mantova, ha saputo che l'Intendenza Provinciale delle Finanze di Mantova intende citarlo in giudizio per il debito contratto con l'Ergastolo di Pizzighettone. Chiede che siano sospesi gli atti giudiziari finché il Magistrato Politico Camerale decida sulla sua richiesta di gratificazione, fondata sullo svolgimento delle mansioni di assistente ai lavori senza salario. Ed alla Intendenza Regia si ordinava "la sospensione degli irreparabili, datane notizia alla Regia Camera de' Conti”. ${ }^{108}$

6 Ottobre 1794: la Camera dei Conti ha esaminato la duplice

104 Ibid., doc. 4574, 5 marzo 1792, p. 107.

105 Ibid., doc. 4674, 21 maggio 1792, p. 221, ma v. anche doc. 4724, 18 giugno 1792, p. 276.

106 BeCCARIA, Atti di Governo, XV [nt. 47], doc. 5697, 2 dicembre 1793, p. 708.

107 BeCCARIA, Atti di Governo, XVI-I [nt. 66], doc. 5873, 24 febbraio 1794, p. 148 s., ma anche BECCARIA, Atti di Governo, XV [nt. 47], doc. 5698, 2 dicembre 1793, p. 709. 108 BeCCARIA, Atti di Governo, XVI-I [nt. 66], doc. 6136, 16 giugno 1794, p. 484. 
richiesta del Donadeo e si dichiara favorevole alla prima (gratificazione), contraria alla seconda (sospensione del giudizio).

Beccaria rileva che anche la Camera dei Conti ha accertato che l'opera svolta dal Donadeo, come direttore dei lavori dei condannati, è stata in effetti utile, comportando un risparmio per la Camera stessa. Essa inoltre riconosce che la somma di L. 830.11.2 proposta dalla R. Intendenza di Finanza per gratificare Donadeo "dal 16 gennaio 1792 retro, non è corrispondente alle sue fatiche" e, poiché il suo lavoro è continuato sino al marzo 1793, ritiene che la somma possa essere aumentata senza però quantificarla. Beccaria ritiene che per i 4 anni di servizio meriti una gratifica di 100 zecchini, avuto anche riguardo al soldo di L. 1.800 che i precedenti direttori dei lavori percepivano. Si potrà consultare la Conferenza Governativa per il condono del debito che pressoché in egual misura il Donadeo ha verso la Camera. Per quanto riguarda la domanda di assunzione in pianta stabile, anche Beccaria è per la negativa: il convincimento è rafforzato dal fatto che $\mathrm{i}$ condannati in Mantova stanno per tornare a Pizzighettone. ${ }^{109}$

13 ottobre 1794: la Camera dei Conti ritiene corretto sospendere gli atti giudiziari contro Donadeo, in quanto il debito è deducubile dalla gratifica. ${ }^{110}$

3 novembre 1794: ultimo atto. La Conferenza Governativa approva la gratifica di L. 1.600 al Donadeo, dalla quale deve essere però dedotto il debito di L 1537.6.8. ${ }^{111}$

Si può anche capire perché il Donadeo, forse presentendo come sarebbero andate le cose, risulti da un documento del 31 marzo 1794 tra coloro che hanno fatto richiesta d'impiego come assistente presso la Casa di correzione di Milano. ${ }^{112}$

Di sovvenzioni, di gratificazioni e di premi, ma anche di pene Beccaria s'interessò praticamente non poche volte. Ma, prima di considerare Beccaria in azione, ritorniamo al Beccaria teorico. Dagli Elementi emergono principi e concezioni che ispirano Beccaria funzionario. In

109 Beccaria, Atti di Governo, XVI-II [nt. 18], doc. 6453, 6 ottobre 1794, p. $851 \mathrm{~s}$

110 Ibid., doc. 6460, 13 ottobre 1794, p. 860

111 Ibid., doc. 6500, 3 novembre 1794, p. 910. Il voto è così redatto: "Corrente. Si eseguiranno le superiori disposizioni. Beccaria Bonesana".

112 BeCCARIA, Atti di Governo, XVI-I [nt. 66], doc. 5960, 31 marzo 1794, p. 263 s. 
quest'opera, per l'appunto, leggiamo: “[...] fine generale e principio insieme reggitore di tutta la politica economia" è quello "di eccitare nella nazione la maggior quantità possibile di travaglio utile", ${ }^{113}$ che dal legislatore, accorto e abile architetto, "non sarà frenata con assoluti e diretti divieti che la rendono più preziosa alla reattiva immaginazione, ma con ostacoli indiretti che deviino a poco a poco dal tumulto e dal disordine [...]". ${ }^{114}$ Del resto, "[...] l'indole universale dell'umana natura, [è] più sicuramente regolata dagli ostacoli che da' divieti; che si precipita ciecamente verso l'interesse presente ed immediato, trascurante il futuro [...]" ${ }^{115}$ Ancora, "Non già che ogni disciplina [delle arti\} debba essere tolta, ma perché è verissimo che le prescrizioni non debbono essere impiegate se non dove sono necessarie; e dove il premio può ottenere il fine voluto dalle leggi, ivi la pena sarebbe dannosa". ${ }^{116}$

Sono, questi, utili suggerimenti che Beccaria teorico offre al legislatore e al funzionario, quindi a se stesso: il saggio architetto deve procedere, operare non imponendo divieti, ma ponendo ostacoli; non infliggendo pene, ma concedendo premi. Gli ostacoli sono strumenti ai quali il legislatore può e talora deve ricorrere per mortificare, per frenare quella forza simile alla gravità che spinge l'uomo a privilegiare il proprio, particolare interesse a discapito dell'utilità collettiva ch'è premessa della comune felicità. Gli ostacoli per essere utili non debbono essere dettati dall'amor di perfezione, ma dalla necessità. In ordine a certe manifatture, particolarmente esposte al pericolo di frodi non immediatamente rilevabili (stoffe colorate, pelli, metalli), utili risultano le leggi che disciplinano le dosi degli ingredienti, i tempi della lavorazione, non solo, ma anche quelle che assoggettano a controllo il manufatto e che contemplano l'impressione di un segno visibile, il bollo, come riconoscimento della sua perfezione. Scrive Beccaria che siffatta imposizione, che si risolve in precauzione, "non sarà mai riputata una violenza ed un legame fastidioso per

113 BeCCARIA, Elementi [nt. 4], p. I, Principj e viste generali, $\$ 17$, p. 35.

114 Ibid., p. I, cap. III, $₫ 38$, p. 74. Con riferimento alla vita dissoluta, seconda causa della rarità delle nozze.

115 BECCARIA, Prolusione [nt. 5], p. 173, ove inoltre si legge: “[...] amante la varietà e la mutazione, ma nel giro delle consuete cose, dagli esempi delle quali è piuttosto guidata che da' ragionamenti; desiderosa del molto agire, ma colla minor fatica possibile; dalla certezza, sia del bene, sia del male, animata e frenata, avvilita dall'arbitrio, e dalla incertezza".

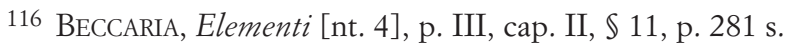


la libertà delle arti [...]; né i buoni manifattori giammai se ne querelano [...] se non quando si volessero troppo pedanteggiare, o si molestassero con inutili formalità, o di questi bolli se ne facesse un articolo troppo oneroso di finanza, o un laccio per fare inciampare in pene pecuniarie i poco avveduti $[\ldots]$ ". Anche il bollo, insomma, per risultare effettivamente utile, doveva essere informato alla libertà. Nel senso che tutti i manifattori dovevano essere liberi di assoggettarsi alle leggi disciplinatrici una determinata produzione, la quale, a riprova dell'osservanza di tali leggi, porterà il bollo. Le merci, mediante il bollo, "avranno la pubblica fede ed autorità garante della bontà e fedeltà con cui sono eseguite”, mentre quelle prive di bollo saranno sottoposte all'esame ed alla valutazione dei singoli acquirenti, correndo il grave rischio della "diffidenza che possono risvegliare mancando di questo solenne testimonio". Beccaria così conclude: "Parmi che un tal mezzo sarebbe più conforme a quello spirito di libertà con cui le arti vogliono essere trattenute, né meno efficace del metodo universale e perquisitorio, perché sufficiente sarebbe a conservare la buona fede dell'esterno commercio; e tutti i buoni e utili manifattori vi si sottometterebbero tanto più volentieri, quanto questa sommessione darebbe loro un vantaggio ed una preferenza sopra i renitenti". ${ }^{117}$

Portiamoci ora, ancora una volta, a Como, 1789. Una pezza di mantino (i.e., lustrino) fabbricata a Como era stata portata a Milano, accompagnata dalla perizia eseguita da Antonio Maria Pizzala, che asseriva "non poterla giudicare imperfetta bensì di sua fattura tollerabile". Sorgono contestazioni da parte della ditta Cossa, per conto della quale era stata fabbricata la pezza oggetto della contestazione, e vengono richieste altre perizie. Sullo sfondo, da tener presente, il disordine dei tessitori e operai comaschi, che si rendeva di giorno in giorno più sensibile, con grave discredito delle manifatture locali e con altrettanto grave danno per i negozianti fabbricatori. Dal confronto delle perizie risulta che quella del Pizzala era dovuta, mi rifaccio al testo, ad "arbitrio" o "imbecillità", meglio la seconda, dal momento che nel voto si legge: "Io voglio credere che il male sia provenuto dal secondo vizio piuttosto che dal primo sapendo io esser egli uomo vecchio e rimbambito né più capace di accedere a una così importante incombenza [...]". Altre pezze della stessa provenienza presentavano identici difetti,

117 Ibid., p. III, cap. IV, $\$ 32$, p. 331-333. V. anche infra, testo corrispondente a nota 143 . 
riconosciuti anche all'estero. Beccaria di fonte a situazione così difficile pensa ad alcune provvidenze: a) invio a Como di uno dei commissari periti milanesi, che esaminasse tutti i telai battenti, riconoscesse le pezze poste in essi e che notasse tutti i difetti che determinavano il loro rifiuto. Pensava inoltre che utilmente si sarebbe potuto contrassegnare le pezze con il sigillo camerale di Como; b) il Commissario, nel caso in cui avesse trovato resistenze nell'espletamento delle sue funzioni, avrebbe potuto far ricorso al braccio della giustizia, "col mettere guardia alle case visitate per ogni occorrenza e per ovviare agli insulti”. Ciò però non doveva essere fatto "che nell'assoluta necessità, bastando che la man forte sia pronta solamente all'occorrenza"; c) la Camera mercantile non doveva più notificare alcuna pezza destinata alla Germania, se prima non fosse stata "visitata" in presenza degli abati e del proprietario, "rigettando assolutamente le difettose ed approvando con un bollo adattato quelle che si riconosceranno di buona qualità". Si imponeva inoltre la necessità di trovare un nuovo perito che "fosse uomo imparziale, intelligente e di petto forte", meglio se forestiero. Se poi ciò dovesse comportare maggior esborso per il salario, poco male, "purché la mira fosse di sciegliere un soggetto abile e non di sussidiare un uomo bisognoso". ${ }^{118}$ Ov'è da notare che i "soccorsi elemosinieri", malvisti da Beccaria a livello collettivo, non debbano trovare applicazione neppure a livello individuale.

Gli ostacoli, dei quali si diceva, possono essere di diversa natura e assumono veste diversa a seconda della materia per la quale si rendeva necessaria la loro instaurazione. L'ostacolo può consistere in un tributo capace di favorire o sfavorire, attraverso esenzioni o aggravii, determinate colture, ovvero di operare, ora come freno, ora come stimolo "alle diverse sorta d'industria, sicché rallentati o accresciuti a proposito, fanno della confusa e molteplice varietà d'interessi un tutto che collima al bene universale della società [...]" ${ }^{119}$

Per quanto riguarda il grano: il venditore, nel caso in cui debba sopportare l'elevato costo di un trasporto lungo e difficile, senza risentire vantaggio alcuno dal rialzo del prezzo determinato appunto dal costo del trasporto, ha tutta la convenienza a vendere il grano all'inter-

118 BeCCARIA, Atti di Governo, XI [nt. 9], doc. 3186, 24 agosto 1789, pp. 550554. Sul Pizzala v. anche ibid., doc. 3302, 26 ottobre 1789, p. 709: "Il Commissario perito Pizzala è assolutamente incapace [...]".

119 BECCARIA, Elementi [nt. 4], p. II, cap. IV, $\$ 35$, p. 174. 
no dello stato. Ebbene, qualora si rendesse necessario intervenire per limitare la libertà di commercio del cereale, ciò si potrebbe fare rendendo artificialmente "lungo e difficile e dispendioso" il trasporto, che "di sua natura" sia facile e breve. Strumenti utili a questo fine sono le gabelle poste ai confini dello stato, in funzione di ostacolo, non già le proibizioni assolute. ${ }^{120}$

Speculari rispetto alle gabelle, sono le gratificazioni. Se queste ultime sono stimolo al commercio, le gabelle, come appena detto, sono ostacoli frapposti al commercio stesso. "Le gabelle sono pagamenti del commerciante al sovrano ed allo stato, quando egli faccia un tale e tale commercio che riesce meno utile; le gratificazioni sono pagamenti del sovrano e dello stato a chi fa tali e tali altri commercj considerati come utili alla nazione. Le gabelle sono allungamenti e difficoltà di trasporti; le gratificazioni, accorciamenti e facilità de' medesimi". ${ }^{121}$

Gabelle e gratificazioni sono, possono, essere strumenti utili per una manovra complessa, sempre che la necessità imponga di scostarsi dal regime di libertà assoluta. "Così di alcuni generi, di cui si voglia ritardare o diminuire l'uscita, ed accrescere ed incoraggire l'entrata, s'imporrà la gabella all'uscita e la gratificazione all'entrata, in modo che il prodotto della prima serva ad introdurre il fondo per l'altra. Parimenti può essere utile d'imporre la gabella all'entrata e la gratificazione all'uscita, allorché siavi bisogno d'introdurre una coltura d'un genere del quale il paese manchi, e nel medesimo tempo ne sia suscettibile". ${ }^{122}$

Il rapporto che lega gabelle e gratificazioni è simile a quello che intercorre tra pene e premi. Quest'ultimo rapporto è fissato in una frase degli Elementi, che già ho avuto occasione di riferire: "[...] e dove il

120 Ibid., p. II, cap. V, $\mathbb{\$} 48$, p. 199 s.: “[...] in questa maniera si avrà il doppio vantaggio di conservare in apparenza tutta la possibile libertà, che animando gli uomini alla fatica e all'industria del commercio si mantiene alacre e vigorosa, e nel medesimo tempo di frenare l'interesse personale tra quei limiti ne' quali divenga una forza combinata con il bene pubblico, non una contraria e distruttiva di quello".

121 Ibid., p. II, cap. V, $\$ 59$, p. 214.

122 Ibid., p. 214 s. E' quanto fecero gli Inglesi con l'Atto di navigazione: “[...] imposero la gabella all'entrata del grano e la gratificazione all'uscita, in quel tempo appunto che il territorio non somministrava grano sufficiente alla consumazione ed andava per la massima parte incolto; perché allora tutti i coltivatori a gara si affaticarono per seminare e raccogliere una derrata così preziosa”. 
premio può ottenere il fine voluto dalle leggi, ivi la pena sarebbe dannosa". ${ }^{123}$ Una massima che riposa su quella che per Beccaria è funzione primaria della legge, la funzione pedagogica: la legge, infatti, deve non già costringere, reprimere, quanto piuttosto educare, guidare. La nazione milanese, del resto, "è docile, ubbidiente, niente facinorosa [...], ma è pigra e si avvilisce facilmente, ha bisogno d'incoraggimento, di una migliore educazione, a cui veramente tendono le paterne sovrane providenze". ${ }^{124}$

E' preferibile dunque che la legge, perché il pubblico interesse prevalga sul privato, faccia ricorso, anziché allo stimolo negativo del timore della pena, a quello positivo consistente nella speranza del premio: "Un altro mezzo di prevenire i delitti è quello di ricompensare la virtù. Su di questo proposito osservo un silenzio generale nelle leggi di tutte le nazioni del dì d'oggi. Se i premi proposti dalle accademie ai discuopritori delle utili verità hanno moltiplicato e le cognizioni e i buoni libri, perché non i premi distribuiti dalla benefica mano del sovrano non moltiplicherebbeno altresì le azioni virtuose? La moneta dell'onore è sempre inesausta e fruttifera nelle mani del saggio distributore". ${ }^{25}$ Ricordo qui che Gian Rinaldo Carli, nominato nel 1765 presidente del Supremo Consiglio di economia, aveva visto accettato il suo "Piano de' premi che annualmente si dovranno assegnare tanto per le

123 Ibid., p. III, cap. II, $\mathbb{S} 11$, p. 282. Anche Melchiorre Gioia riponeva grande fiducia nel premio, nella ricompensa, più che non nella pena: "Si possono certo reprimere i delitti colle pene, ma più sarà forte la ricompensa destinata alla virtù, più scemerà la necessità di reprimere colle pene i delitti”. Due erano le motivazioni di fondo che lo inducevano a collocarsi nel solco del pensiero beccariano, una di economia processuale, l'altra di politica economico-criminale: "1. Perché per punire l'ommissione di un servizio fa duopo essere sicuri che l'individuo aveva il potere di renderlo, e ragioni legittime non s'univano a dispensarlo; è quindi necessario un processo talvolta difficile, sempre costoso, e per lo più molesto a molti; 2 . Se operiamo solo per timore della pena, noi eseguiamo quegli atti solamente che bastano per evitarla, ad imitazione degli schiavi. All'opposto gli sforzi eccitati dalla speranza della ricompensa, come negl'operaj liberi, sono molto maggiori, soprattutto allorché è libero il campo dei concorrenti”. M. GioIA, Del merito e delle ricompense. Trattato storico filosofico [...], in Nuovo prospetto delle scienze economiche [...]. Tomo VIII. Serie II. Trattati pratici, I. Filadelfia 1819, p. 175 s.

124 BeCCARIA, Atti di Governo, IX [nt. 19], doc. 1846, Codice generale (relazione, [giugno 1787]) Brevi riflessioni intorno al Codice generale sopra $i$ delitti e le pene per ciò che riguarda $i$ delitti politici, pp. 477-490, in particolare, p. 484.

125 BECCARIA, Dei delitti e delle pene [nt. 6], $\mathbb{X}$ XIV Ricompense, p. 126. 
accademie quanto per l'agricoltura, arti e manifatture, esaminando nel tempo medesimo, e consultando i modi onde poter sollecitamente redimere il dazio della seta greggia, da destinarsi principalmente a quest'oggetto" [Apih, 1973, p. 244].

Dei principi espressi in sede teorica, come si è or ora ricordato, Beccaria fece pratica attuazione. Proprio rivolgendosi a Carli, dal quale era stato incaricato nel 1771 "di invigilare in questa Regia Zecca alla comandata operazione di battere centomille lire, parte in venti soldi e parte in dieci, e principalmente di accudire alla necessaria restaurazione degli utensigli di Zecca che, come è noto a Vostra Eccellenza, si sono trovati in pessimo stato, e di tenere in soggezione gli operari”, Beccaria si rivolgerà per mettere in luce i meriti del regio assaggiatore Brusasorci, che si era distinto per "l'esattezza, attività perizia e zelo il più sincero" nel sostenere le fatiche del suo lavoro e del "Legnani [che] non ha risparmiato di tutta la sua attenzione e presenza", avendo svolto non solo il suo ufficio di "prevosto agli operari, ma anche le parti di amministratore e ragionato". L'uno e l'altro, pertanto, meritavano "in via di premio e di salario insieme venti zecchini per ciascheduno". E meritevole di premio era risultato anche Giuseppe Saint-André, "ch'aveva ideato, eseguito e messo in lavoro in Mantova un ingegnoso ordigno in cui molte donne lavoravano contemporaneamente ed equabilmente lino, cotone, lana, bavella". ${ }^{126}$

Nel 1790, sui primi di settembre, Beccaria era a Como per fronteggiare la preoccupante sollevazione dei tessitori serici disoccupati, e molti erano stati i provvedimenti proposti o di già attuati: erano stati incarcerati sette tessitori arrestati in osteria. Provvedimento legittimo perché attuava un avviso dell'Intendente politico - il già sindaco fiscale

126 BeCCARIA, Atti di Governo, XVI-II [nt. 18], Addenda, doc. 8.1, 29 ottobre 1771, p. 975 s.; IX [nt. 19], doc. 1689, Attività del Dipartimento III nel 1786 (relazione, 31 marzo 1787), p. 232. Ricordo anche, toccando tutt'altro tema, lo spietato utilitarismo economico, al quale, secondo un atteggiamento piuttosto diffuso, si ispirava Carli "[...] una mala vita fa più esempio di una mala morte [...]"; "Non si propone la prigione perpetua, perché inutile, ma una vita schiava a servizio della società. Vita miserabile e d'esempio e nel medesimo tempo utile alla società. Io che sono amico degli uomini, la penso così". Gian Rinaldo Carli a Gravisi, 29 aprile 1765, in Baccio Ziliotto, Trecentosessantasei lettere di Gian Rinaldo Carli capodistriano, cavata dagli originali ed annotate, in Archeografo triestino, terza serie, voll. IV-VII. Trieste 1909-1914. Cito da APIH, 1973, p. 224. 
Giuseppe Pellegrini [Mozzarelli, 1990, pp. 79, 82, 84] - che prevedeva la carcerazione per quei tessitori che fossero stati colti "uniti tra di loro in maggior numero di tre". Ora, però, tornata la quiete, era bene lasciar cadere siffatto provvedimento "che poteva dar luogo a molti arresti incongrui ed odiosi", dal momento che "[...] quei rimedi straordinari che sono proficui nelle occasioni straordinarie divengono, restituito l'equilibrio, perniciosi, oltrecché danno ansa ai cervelli torbidi di credere che si abbia paura di loro". Era bene, invece, conservare il provvedimento politico là dove perseguiva "i tessitori oziosi in qualche numero uniti a questuare talvolta per le strade in un modo risoluto ed esigente [...]".

Assai utile sarebbe stato, secondo Beccaria, l'elenco dei tessitori - il loro numero era rilevante: circa seicento i "telari operosi", almeno duecentocinquanta i "tessitori oziosi" - in quanto, se ben fatto, poteva essere "la base di tutte le provvidenze future". Potevano essere distinti in tre classi, diversamente disciplinate: $\mathrm{i}$ tessitori che attualmente hanno un lavoro, ove "facessero i molesti con importune questue o con promuovere soccorsi e fazioni”, avrebbero dovuto essere colpiti da "un più serio castigo degli altri"; per i "tessitori accidentali", vale a dire coloro che non sono abitualmente occupati come tali, ma che, piuttosto, esercitano un altro mestiere (per esempio, il barcaiolo), ovvero che "si sottraggono alla campagna per il più dolce e libertino vivere cittadinesco", nessuna provvidenza era prevista, ma dovevano inesorabilmente essere "rimandati al primo loro mestiere"; da ultimo, quelli "provetti nel serificio", che altro mezzo non hanno per vivere "essi e le loro famiglie, e questi sono quelli che meritano speciale provvidenza e compassione". Siffatto elenco poteva in effetti risultare utile anche per un provvedimento di fondamentale importanza, che tocca una materia per la quale, come già sappiamo, Beccaria mostra particolare sensibilità. "Tutta la tendenza dei fabbricatori comaschi [...] è quella di avere gratificazioni e soccorsi gratuiti di danaro dalla camera, coi quali soccorsi fabbricando con vantaggio sopra gli altri fabbricatori dello stato, hanno il doppio vantaggio di fabbricare a preferenza a minor prezzo e con maggior guadagno [...]" [Caizzi, 1957, p. 31 s.]. Occorreva opporsi a questa tendenza, a questa "sagace speculazione" che è stata "forse la principal sorgente delle passate turbolenze", col non accedere ad alcuna domanda di soccorso da parte dei fabbricatori e col diminuire indirettamente il sovrabbondante numero di tessitori, tra i quali, mediante la concorrenza, sarebbero stati respinti coloro che "sanno e possono abbracciare un altro mestiere". Coloro che non hanno "altro scampo a vivere che il serificio" e che non trovano occupazione 
presso i fabbricatori comaschi potrebbero lavorare in Como per conto di imprenditori milanesi, incoraggiati coll'indennizzo delle spese di mantenimento di un institore, nonché di quelle del trasporto a Milano delle pezze fabbricate in Como. Si trattava di uno "spediente" in cui Beccaria riponeva fiducia: "avrebbe il vantaggio di dare impiego a quei tessitori che lo meritano, di eludere la speculazione comasca, e di emancipare il Consiglio e il fondo del Commercio di dover sempre accorrere con sussidii alle troppo frequenti sospensioni comasche, e potrebbe forse introdurre in Como esempio di una maggior varietà e bontà di travaglio $[\ldots]$ coll'introdurvi il lavoro delle saglie, de' veri lustrini, degli amoerre, de' rasi, riducendosi presso che tutte la fabbricazione comasca a' soli mantini, e questi per lo più scadenti e viziosi in qualche parte". Era inoltre uno "spediente" ben più "plausibile" e "giovevole" rispetto ad un altro provvedimento che si aveva in animo di prendere: quello di "fare ben presto una leva forzata dei più robusti inquieti fra questi tessitori per consacrarli al militare". La contrarietà era determinata dal fatto che si trattava di un rimedio non giusto, perché non necessario. Beccaria sottolineava: "Un tale ripiego sarebbe certamente sbrigativo ma violento ed ingiusto per un gran numero di essi”. ${ }^{127}$ Sul binomio giustizia-necessità riferito alla pena ritornerò. Per ora mi limito a ricordare come le parole di Beccaria ora riferite risuoneranno ancora, nella loro sostanza, quattro anni più tardi, il 24 gennaio 1792, quand'egli, insieme con Risi e Gallarati Scotti manifesterà il suo pensiero intorno alla pena di morte in seno alla Commissione istituita da Leopoldo II, rimanendo in minoranza: la pena di morte, se "è la più sbrigativa per disfarsi dai rei, non è la più conveniente per reprimere i delitti”, sono le parole col quali si conclude il voto, che racchiude, appunto, anche il pensiero beccariano. ${ }^{128}$

Della difficile situazione economica e sociale vissuta dalla città comasca Beccaria aveva già fatto conoscenza alcuni anni prima, quando fu chiamato a pronunciarsi, raccolti i rapporti delle Camere mercantili di Cremona, Mantova, Como e Milano [Caizzi, 1957, p. 27;

127 BeCCARIA, Atti di Governo, XII [nt. 62], doc. 3838, 17 settembre 1790, pp. 531-540 Ragguaglio fatto al Reale Consiglio di Governo dal Consigliere Marchese Don Cesare Beccaria Bonesana delle operazioni da esso eseguite in Como affine di stabilire la pubblica tranquillità di quel paese.

128 BECCARIA, Atti di Governo, XVI-II. Appendice Voto degli infrascritti individui [...][nt. 18], p. 972. V. retro, testo corrispondente a nota 18. 
Caizzi, 1968, p. 193 s.], intorno ai sedici articoli di cui si componeva un "editto sulla disciplina degli operai del setificio". Alcuni di questi articoli erano inutilmente troppo coattivi e quindi da respingere. Si pensi al primo articolo: "Che ogni lavorante o garzone che, senza legittima causa, mancherà di essere al suo travaglio ne' giorni feriali ed alle ore stabilite debba essere licenziato". Applicato con rigore, come dovrebbe essere ogni provvedimento normativo, due erano le conseguenze prevedibili. Il datore di lavoro al fine di non perdere operai abili, sì, ma non sempre assidui al lavoro, non avrebbe, egli stesso, applicato la legge. Seconda conseguenza: "l'uso di oziare il lunedî" era un inconveniente diffuso, riscontrabile "in tutte le nazioni, anche più attive e industriose della nostra". Del resto, il pretendere "un lavoro forzato il lunedi”" avrebbe significato ottenere un lavoro cattivo e, in buona sostanza, in contrasto con l'interesse dello stesso imprenditore. Ecco, allora, la necessità che entri in campo la libertà, insieme con l'interesse. "Facciano i fabbricatori delle convenzioni coi loro lavoranti quali convengono al proprio interesse, e si potrà meglio ottenere dall'obbligazione di un contratto di un uomo libero ciocché non si ottiene, se non male e quasi mai, dalla forza usata contro di uno schiavo".

Un altro articolo (art. VI) proibiva al fabbricante di concedere a lavoranti e garzoni alcuna anticipazione o sovvenzione: a costoro doveva essere corrisposta solo la somma che avranno guadagnato. Obiezioni: senza sovvenzioni sarebbe stato difficile attrarre lavoranti forestieri "più abili e attivi di quello che lo siano i nazionali naturalmente"; era una legge che offendeva troppo direttamente il diritto di proprietà, "che non deve togliersi che per motivi gravissimi e per una necessità indispensabile al mantenimento della società civile". E questo non era di certo il caso in questione.

L'art. XIV si riferiva ai "sovvertitori degli operari altrui". Certo, occorreva impedire che i fabbricanti, con promessa di maggior guadagno, attraessero nella propria fabbrica quegli operai altrove occupati, ma non condivisibile era la pena di 5 anni di galera, che doveva essere irremissibilmente scontata, comminata dall'editto "a chi tenta l'emigrazione delle arti e manifatture". Si trattava di una pena contraria alla natura del delitto, in quanto il suo autore contravveniva sì "a una legge di uno Stato", ma non alle "leggi della natura, per le quali offese sembrano doversi serbare le pene afflittive e infamanti", qual era quella della galera. Ritenendo, pertanto, solo una parte dell'articolo in esame si eliminava ciò che in esso vi era di odioso, e, in pari tempo, si conser- 
vava "il più che si può di una legge vecchia e altronde non eccessiva e giusta, trattandosi di veri seduttori ed ingaggiatori maliziosi”. La pena proporzionata alla gravità del delitto era quella che, contemplata da un editto del 1764, prevedeva la sanzione pecuniaria "di 25 scudi e anche maggiore e corporale secondo le circostanze de' casi”, pena utile, questa, e conforme alla natura del delitto. L'articolo successivo dell'editto, ed è l'ultimo che qui considero (art. XV), contemplava l'assegnazione annua di tre medaglie per coloro che nel lavoro avessero profuso, distinguendosi, diligenza e buona condotta. L'obiettivo, condiviso da Beccaria, era quello di assicurare la più puntuale osservanza della disciplina edittale, contribuendo in pari tempo ad assicurarne la dolcezza. ${ }^{129}$

Intorno alla concessione di premi Beccaria aveva espresso invero qualche incertezza, quando, sempre in ordine alla critica situazione comasca, si era pronunciato intorno all'opportunità stessa di approntare e di promulgare un editto per affrontarla e risolverla. L'Intendente politico di Como, che si faceva interprete della volontà di esponenti della Camera mercantile, di "qualche altro dei principali negozianti fabbricatori, Monsignor Vescovo e il signor conte Giovio", sosteneva la necessità di una "legge disciplinaria". Anche Beccaria era convinto dell'opportunità di qualche provvidenza: "la difficoltà consiste nel scegliere la meno vincolante e la meno pericolosa, e scelta poi che sia, sostenerla col dovuto rigore". Tenendo sempre presente che, se i rimedi dolci sono lenti, quelli violenti sono pericolosi: una pericolosità che riposa su di una considerazione di natura economica. Una legge troppo severa, troppo vincolante può determinare in tempi brevi l'emigrazione degli operai con l'effetto, benefico, di allontanare i soggetti "cattivi e poco operosi, la perdita de' quali sarebbe supplita dal maggior travaglio di quei che rimangono", ma con l'effetto anche, assolutamente non benefico, dell'aumento del costo della mano d'opera. Un provvedimento eccessivamente aspro avrebbe inoltre potuto "allontanare gli operari forestieri dal venire a cercare lavoro e domiciliarsi in paese".

L'Intendente politico avanzava inoltre tre proposte, che Beccaria attentamente valuta. Quanto a quella che vietava ai fabbricanti di aumentare il salario agli operai, premesso che "dovrebbe essere inte-

129 BeCCARIA, Atti di Governo, IX [nt. 19], doc. 1770, 14 maggio 1787, pp. 372 388. Intorno al rapporto fabbricanti-operai, v. anche doc. 1689, Attività del Dipartimento III nel 1786 (relazione, 31 marzo 1787), p. 237. 
resse degl'intraprenditori di non farlo ordinariamente", Beccaria era del parere che un divieto espresso per legge fosse troppo vincolante e dannoso, "troppo angustiante il commercio". In quanto tale, forte, fortissimo era il rischio dell'elusione del provvedimento. La seconda proposta concerneva le penali "le quali egli fa giungere fino alla Carcere, alla Berlina". Anche in questo caso Beccaria rileva come eccessiva fosse la severità adottata: occorrendo non confondere "i vizi coi delitti, sono di gran lunga preferibili le multe alle pene afflittive, e soprattutto alle infamanti". L'Intendente proponeva ancora che l'asprezza delle minacce venisse temperata colla promessa di premi "agli esatti e assidui operari”. Qui Beccaria manifesta quella incertezza intorno ai premi alla quale ho fatto poco fa riferimento. Infatti, ove si fosse largheggiato nella loro concessione, troppo elevata sarebbe stata la somma di danaro impiegata; ove si fosse voluto tener troppo stretta la mano, sarebbero sorti sospetti di parzialità, scatenando le lamentele dei delusi. E non sarebbero, da ultimo, mancate le difficoltà nella determinazione e valutazione di quei "minuti fatti" utili ad assicurare la corretta ed equa distribuzione dei premi. A Beccaria sembrava che, nel caso, "sufficiente premio" fosse quello del maggior utile che i lavoranti avrebbero tratto dal maggior lavoro. ${ }^{130}$

Da Beccaria il premio era configurato, in generale, come una ricompensa. Le arti s'incoraggiano premiando il lavoro svolto: "Dunque premiar l'opera già fatta, sarà la massima più salutare e il mezzo più efficace a promuovere le manifatture. Il premio è di uno solo, ma l'emulazione è di molti [...]". ${ }^{131}$ Si tratta di una massima che trova coerente riscontro nella prassi: "Il premiare la cosa fatta è sempre stato il mezzo più efficace ad incoraggire le cose da farsi, quando sia adoperato con imparzialità e costanza, ed è ad un tempo quello che è il meno rischioso, ed imbarazzante per il Regio erario, o se i premii sian piccoli, ma frequenti anche preso la cosa in grande, ed alla lunga forse il meno dispendioso [...]". ${ }^{132}$

130 Ibid., doc. 1654, 12 marzo 1787, Brevi riflessioni che si subordinano dal Relatore per le superiori determinazioni, pp. 171-180.

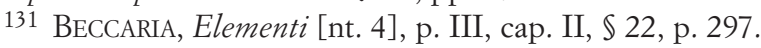

132 C. BECCARIA, Relazione sui mezzi più idonei per promuovere la costruzione di case rustiche nel Mantovano. 22 giugno 1789. Il documento è pubblicato in CANETTA, 1973, doc. n. 16, p. 159 s. 
Nello stesso modo Beccaria pensava intorno ai privilegi, purché non fossero esclusivi. Infatti, se dai più il privilegio era ritenuto utile al promovimento delle arti, in esso Beccaria coglieva un ostacolo. I privilegi possono in realtà essere davvero utili quando non siano concessi in esclusiva ad alcuni a preferenza di altri, "[...] quando siano concessi non alle persone direttamente, ma alle azioni conformi al pubblico bene, in modo che a chiunque sia aperto l'adito del godimento del privilegio, perché sia in suo potere di fare quell'azione, a cui quello va annesso". Pare non vano soffermare un poco l'attenzione su quanto Beccaria scrive nell'argomentare la sua avversione per quei privilegi "che si accordano a tali manifattori contro tutti quelli che potrebbero intraprendere il medesimo lavoro". Le arti, invero, "come le cose tutte, non prosperano quasi mai nelle mani di un solo. Tale è la legge eterna che contribuisce a legare gli uomini in società". L'arte, che rimane nelle mani di uno o di pochi manifattori, non solo rimane "sempre languida e imperfetta", ma procura arricchimento non già alla nazione, quanto piuttosto al singolo privilegiato. Grande è il vantaggio che deriva dalla libertà per ciascheduno di professare o esercitare l'arte preferita: v'è emulazione nella tensione a perfezionarla, e concorrenza nell'obbiettivo di ridurne il prezzo. "Dippiù, non ho difficoltà di qui ripetere, perché importante, ciò che altrove ho accennato, cioè che la concorrenza dei manifattori abbassando il prezzo della manifattura e perfezionandone l'opera, aumenta di più la ricerca e lo spaccio, di quello che non scemi alla lunga il profitto di ciascheduno in particolare, supposto che questi avesse il privilegio esclusivo, il quale se esclude gli altri dall'esercitare un'arte simile, esclude anche ed aliena una parte dei compratori dal procacciarsi le produzioni di quella”. Contrarietà netta, dunque, contro i privilegi esclusivi, contro le privative. Piuttosto che accordare privative, la "sana politica" vuole che si rimanga privi di una qualsivoglia arte. Preferibile è fissare premi e gratificazioni in favore di colui che, per primo, avrà il coraggio di "arrischiare un'intrapresa". Al contrario dei privilegi esclusivi, che sono d'intralcio al fiorire e al progredire delle arti, i premi le favoriscono, assicurandone l'espansione. Dal momento che l'uomo "è meno mosso dall'ambizione che dalla speranza di un bene più reale ed immediato", i premi costituiscono "i più efficaci animatori delle arti, e faranno incurvare l'inerte alla fatica, e renderanno sagace l'industrioso nell'inventare e finire le opere sue".

Talora la manifatture ricevono somme che "la generosa munificenza del sovrano somministra a chi si esibisce a sostenere un'intrapresa". Anche questo risulta per Beccaria uno strumento non utile per 
incoraggiare le arti. Ciò per due motivi: chi riceve un capitale risulta troppo avvantaggiato rispetto agli altri, impossibilitati a sostenere la concorrenza. In buona sostanza, s'introdurrebbe non una manifattura, ma un manifattore. Inoltre, chi riceve un capitale "cercherà di campare sopra di esso contentandosi di esibire un'apparenza di travaglio, più per conservarsi il diritto di prolungare la restituzione e di chiedere nuovi soccorsi, che per corrispondere con lealtà alle benefiche mire del sovrano". La conclusione è quella che già si è avuto occasione di ricordare: "Dunque premiar l'opera già fatta, sarà la massima più salutare ed il mezzo più efficace a promuovere le manifatture. Il premio è di uno solo, ma l'emulazione è di molti, e la speranza che è uno dei più grandi agenti dell'uomo socievole, mette in fermento l'interesse privato di ciascheduno; e il profitto che risulta da questa prima spinta è tale, che in seguito quasi senza il premio la manifattura si dilata e rinvigorisce". ${ }^{133}$

Nella prassi poi, anche in una situazione di preoccupante disoccupazione, è bene non pensare a premi e gratificazioni, è bene non ricorrere ai "soccorsi elemosinieri", i quali in realtà sono "perniciosi se non sono un compenso del lavoro o un sussidio alla fisica potenza". Era opportuno, piuttosto, pensare ad un lavoro che risultasse utile, oltre che alla collettività, anche a coloro che ne erano privi, in modo tale che potessero guadagnare di che vivere. ${ }^{134} \mathrm{Nel}$ caso, la bonifica di un luogo malsano - l'attenzione era di frequente rivolta alla necessità e utilità di "rendere asciutta la palude presso il Borgo Vico", vale a dire il prato Pasquè - rispondeva a questa duplice esigenza. ${ }^{135}$

133 BECCARIA, Elementi [nt. 4], p. II, cap. V, $\$ 57$, p. 212; p. III, cap. II, $\$ \$ 18$ 22, pp. 289-298. Nello stesso senso, fondamentalmente, anche Pietro Verri nelle sue Meditazioni sull'Economia politica (Livorno 1772). Testo a cura di R. PESTARINO. Commento di P.L. PORTA, in ID., Scritti di economia, finanza e amministrazione a cura di G. Bognetti, A. Moioli, P.L. Porta, G. Tonelli, vol. II, tomo II dell'Edizione Nazionale delle Opere, Roma Milano 2007, $\mathbb{X}$ X De’ privilegi esclusivi, pp. 441-443.

134 BeCCARIA, Atti di Governo, IX [nt. 19], doc. 2061, 22 ottobre 1787, p. 755.

135 Ibid., doc. 2099, 12 novembre 1787, pp. 803-805; doc. 2140, 3 e 17 dicembre 1787, pp. 854-864: "L'opera, proposta fino da principio, si era quella di abbassare la foce del torrente Cosia collo scavo della ghiaia per rovesciarla sull'adiacente prato detto Pasquè, reso dalle ordinarie e straordinarie escrescenze del Lago paludoso, di modo che, principalmente nell'estate, infesta l'aria di Borgo Vico, fino sotto le mura della città" (p. 856); doc. 2147, 17 dicembre 1787, p. 873 s., ove si specifica che l'area del prato era di 70 pertiche. 
Si era nel 1787: non pochi anni dopo, nel 1794, Beccaria si mostra fermo nel suo convincimento. Un centinaio di abitanti di Biumo, castellanza di Varese, avevano vivacemente protestato presso il Deputato del censo per "l'eccessiva carezza dei grani, e segnatamente del melgone". Dai Deputati dell'estimo si era pensato di distribuire, al fine di acquietare i disordini, delle elemosine straordinarie a carico dei fondi dell'Ospedale. Altre, secondo Beccaria, dovevano essere le vie da seguire per risolvere la situazione, certamente non quella di impiegare le entrate dell'Ospedale per un fine diverso da quello istituzionale, il soccorso e la cura degli infermi. E dunque: sollecitare i proprietari terrieri a soccorrere i "terrieri miserabili"; impiegare costoro, a preferenza di persone estere, nei lavori di riattamento della strada provinciale che da Varese porta al confine comasco; "sentire di nuovo quei Deputati dell'Estimo se nel distretto territoriale della comunità di Varese o nel vicinato si potesse intraprendere qualche utile opera pubblica, benché non fosse d'istantanea necessità, all'effetto di far travagliare le mentovate persone miserabili [...]". ${ }^{136}$

Il premio poteva anche occasionalmente risolvere problemi di aprovvigionamento. Nel 1790, per fare un esempio, si era reso difficoltoso il sopperire al fabbisogno di due generi di "somma necessità", quali erano il carbone e la carbonella. Inopportuno appariva a Beccaria il partito di aumentare la meta - "incomparabilmente sarebbe stato maggiore l'utile dei conducenti ed il danno del pubblico se si fosse preso il partito di accrescer di qualche poco la meta a detto genere"-; senza dubbio più vantaggioso quello di provvedere alla "rimozione de' Ghiaiati", che rendono difficile il trasporto lungo il Ticino, duplicando la spesa, ma di non facile e immediata soluzione, in quanto "ciò dipende dai concerti colla $\mathrm{R}$. Corte di Torino". "Non saprebbe quindi il Relatore, nelle attuali circostanze, che adottare il partito dei premi", cioè di "allettare con qualche premio i conducenti di questo genere" così necessario per superare le difficoltà del trasporto. Si trattava comunque di un espediente, che doveva trovare applicazione "unicamente quando il bisogno lo richiede": Beccaria temeva che "l'esempio di quest'anno" divenisse "pericoloso pei susseguenti". ${ }^{137}$

136 BeCCARIA, Atti di Governo, XVI-I [nt. 66], doc. 5836, 10 febbraio 1794, p. 105 s.; doc. 5891, 3 marzo 1794, pp. 169-172.

137 BeCCARIA, Atti di Governo, XII [nt. 62], doc. 3576, 15 febbraio 1790, p. 162; doc. 3878,25 ottobre 1790 , p. 591 s.; doc. 3658,3 maggio 1790, p. 270 s. (osservazioni e riscontri al n. 422). 
Di premi, di gratificazioni Beccaria parla anche con riferimento a coloro che sono investiti di una pubblica funzione: il teorico sembra, nel caso, sopraffatto dal funzionario, dal Beccaria pragmatico. Da un lato, infatti, eccolo pensare che "interessare i custodi nell'invenzione" dei beni contrabbandati, risultava "metodo troppo abusivo", ${ }^{138} \mathrm{ma}$, dall'altro, "Per animare i Ricettori, Guardie di finanze ed uomini d'armi a ben invigilare contro l'estrazioni clandestine de' grani, gioverebbe forse lasciare a loro profitto non solo il grano confiscato, ma parte della penale ancora". ${ }^{139}$ Si era nel 1781 e, circa dieci anni dopo, nei lavori della

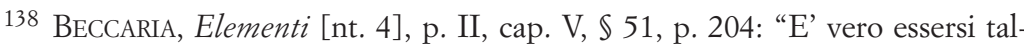
volta usato d'interessare i custodi nell'invenzione, ma questo metodo è troppo abusivo per chi pensa allo spirito della legge e del divieto, il quale consiste a far sì che la merce non esca, non vi siano invenzioni e non vi siano patti co' custodi, onde divenga un oggetto di rendita e di privativa ciò che è un oggetto di sicurezza e di precauzione".

139 C. BeCCARIA, Atti di Governo (serie II: 1778-1783), a cura di R. CANETTA, vol. VII dell'Edizione Nazionale delle Opere diretta da L. FirPo e G. FrAnCIONI, Milano 1990 (d'ora innanzi, BECCARIA, Atti di Governo, VII), doc. 688, Nuovo Piano annonario (relazione, 15 settembre 1781), n. 2 Riflessioni alle Idee relative alla formazione del Piano annonario ed alle osservazioni e quesiti rimesse con lettera di Governo 26 marzo 1781, settimo, p. 558 s. Nella Circolare ai Regi Cancellieri delle pievi montuose, a firma di Verri e di Beccaria, si legge che le pene previste nell'editto 9 maggio 1784 non valevano a raffrenare le contravvenzioni consistenti "nell'arbitrario taglio de' boschi comunali, anche per opera degli stessi comunisti" e che, pertanto, il Tribunale aveva stabilito di concedere in favore dei Regi Cancellieri la metà delle multe di spettanza delle comunità, al fine di animarli "ad invigilare sull'osservanza degli ordini in tal proposito [...]" (BECCARIA, Atti di Governo, VIII [nt. 19], doc. 1236 (minute di consulta e di circolare, 12 dicembre 1785 e 16 gennaio 1786), pp. 551-554, in particolare, pp. 552, 554. Le pene previste dall'editto richiamato (lo si legge in minuta, ibid., doc. 88517 marzo 1784, pp. 21-27) consistevano nella perdita della legna tagliata e nel pagamento di uno scudo "per ogni fascio o carica che vi avrà tagliata", qualora l'illecito fosse stato commesso nei boschi di uso comunale. Qualora fosse avvenuto nei boschi di riserva, la sanzione pecuniaria ascendeva a 2 scudi (nn. 5, 7, p. 22 s.). Sul diverso regime "dei boschi che dovranno essere di riserva o d'ingaggio e di quelli che dovranno servire per uso focolare e per pascolo del bestiame", cfr. ibid., doc. 1112, 9 aprile 1785 Istruzioni sul miglioramento de' boschi e fondi comunali della Valsasina (pp. 357-360); Istruzioni sul miglioramento de' boschi e fondi comunali della pieve di Porlezza (pp. 361-364) [Trezzi, 1979, pp. 271-279]. Ambedue le Istruzioni sono a firma di Verri e di Beccaria. Melchiorre Gioia prenderà in considerazione l'editto 9 maggio 1784 -così come quello del 16 gennaio 1786, che, per quanto "più sensato", del pari non ebbe "la debita esecuzione [...]” (M. GIOIA, Sul Dipartimento del Lario discussione economica, Milano 1804 , p. 84) - per esprimere il suo assai severo giudizio: "Esistono varj regolamenti sì 
commissione istituita da Leopoldo II per la progettazione della riforma del sistema criminale lombardo venne espressa la convinzione che era bene assicurare ai custodi e alle guardie il diritto di partecipare alle multe per i generi contrabbandati ed al frutto delle invenzioni come stimolo ad esercitare con zelo e diligenza la loro funzione [Massetto, 1994, p. $521 \mathrm{s.}$. Beccaria ribadiva siffatta impostazione a proposito della figura dei campari così come prevista nel Piano della caccia. Proprio con riferimento a costoro, egli pensava che "L'aumento poi dell'onorario da pagarsi dai contravventori può essere ad un tempo di qualche compenso alla modicità del salario e di stimolo all'esatta e diligente custodia della regalia". ${ }^{140}$

Poniamo ora mente al Dei delitti e delle pene. Il $₫$ XXXVII configura un premio che assume natura del tutto particolare: non consiste nella partecipazione ad una multa o ad una invenzione, nella concessione di moneta, erosa o non che fosse, o di una medaglia, magari in oro zecchino, ma in qualcosa che sotto il profilo penale è per l'interessato di grandissimo momento, in quanto involge la sua libertà, di più, la sua

consuetudinarj che scritti per la conservazione de' boschi, e segnatamente quello del 9 maggio 1784. Tutti questi regolamenti non hanno mai prodotto altro effetto che quello delle leggi dirette, cioè spese per farle eseguire, costante trasgressione; né la cosa poteva altrimenti succedere. Difatti I. ${ }^{\circ}$ la legge stabilisce delle pene pecuniarie contro le persone che vanno a tagliar legna ne' boschi comunali di riserva; ora questa gente è quasi sempre nell'impotenza di pagarle; quindi $2 .^{\circ}$ i cancellieri censuarj e gli agenti municipali sia per compassione, sia per risparmiare una pubblica spesa, sia per non farsi degli inimici, lasciano in riposo la legge e i trasgressori; $3 .^{\circ} \mathrm{i}$ denunciatori particolari sicuri di non ricevere parte della multa, vedendo l'indifferenza delle autorità, s'astengono dalle denuncie per non gettarsi in qualche imbarazzo. Quindi la distruzione non limitata dalla legge si estende fin dove s'estendono le forze fisiche de' comunisti moltiplicate pel disordine de' tagli" (ibid., p. 83 s.).

140 BeCCARIA, Atti di Governo, XI [nt. 9], doc. 2851, 19 gennaio 1789, pp. 3686. Sul punto, p. 70, nonché p. 59 s.: "Quest'onorario [dovuto dal contravventore al camparo] non è espresso nella grida quanto debba essere, ma nella tariffa, perché l'editto suppone che debba essere detratto a favore de' campari dal totale della multa. Quando però segue composizione, come quasi sempre si suole comporre in una somma oltre l'onorario, che è di lire sette per ogni invenzione di cacciatore con lo schioppo e di quattro di cacciatore senza schioppo, essendosi ridotte le multe a minor somma, si può aggiungere alla multa, che è di ragione della Camera, anche l'onorario, che potrebbe portarsi indistintamente a due scudi per incoraggire sempre più i campari, scarsamente pagati, alla dovuta vigilanza”. 
stessa vita. Il premio, infatti, può consistere nell'impunità promessa "a quel complice di grave delitto che paleserà i suoi compagni”. E Beccaria, "cui certo non mancava lo spirito dell'economista" - come ebbe a rilevare un penalista positivo tra i più storicamente sensibili qual è Mario Pisani, proprio qui, in questa sala nell'ottobre del '97 [Pisani, 1998, p. 50] - ponderò "inconvenienti" e "vantaggi" di tale "spediente". E dopo aver ponderato, sia pur preda al rimorso ed al tormento, opta per la soluzione che privilegia l'impunità del delatore, rispetto a quella del suo silenzio, che poco o nulla giova al bene pubblico. Risultava, inoltre, per lui preferibile una legge generale che promettesse "tale impunità al complice palesatore di qualunque delitto", legge che doveva provvedere anche al bando del delatore stesso, rispetto "ad una speciale dichiarazione in un caso particolare"[Vassalli, 1990, p. 31 s.; Neppi Modona, 1990, p. 92].141

Di certo, tentennamento alcuno Beccaria non ebbe nel respingere l'utilità di "mettere a prezzo la testa di un uomo conosciuto reo ed armando il braccio di ciascun cittadino farne un carnefice". Con la taglia, ché di questo si tratta, la nazione mostra tutta la sua debolezza: "chi ha la forza per difendersi non cerca di comprarla”. Dunque, espediente di una nazione debole è quello di spargere diffidenza tra i consociati anziché sviluppare i legami di amicizia, la fiducia tra gli animi, di per sé "sospettosi, degli uomini"; di corroborare, anziché di mortificare e dissolvere quella potentissima forza disgregatrice del tessuto sociale che e è l'egoismo. Insomma, si tratta di uno strumento configurabile, nel quadro della "morale pubblica" che è piegata "ad ubbidire alla privata", come immorale: uno strumento che non deve essere impiegato, ma assolutamente respinto in quanto si oppone "a questa così necessaria riunione della morale e della politica" - tra di loro distinte, certo, ma accomunate dal medesimo fine al quale tendono -, "a cui gli uomini dovrebbero la loro felicità, le nazioni la pace, e l'universo qualche più lungo intervallo di tranquillità e di riposo ai mali che vi passeggiano sopra". ${ }^{42}$

La propensione che Beccaria manifesta per il premio, tanto nella teoria quanto nella pratica, viene ad assumere un ruolo assai importante nella sua concezione della legge e nella sua filosofia della pena, con-

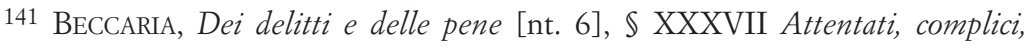
impunità, p. 114 s.

142 Ibid., \$ XXXVI Della taglia, p. 113 s. 
cezioni e filosofia che in modo netto e chiaro si riflettono, propagandone gli effetti, nel settore della pubblica economia [Di Simone, 2000, p. 60 s.]. Lasciamoci guidare da una frase degli Elementi di economia pubblica, che è rivelatrice dell'idea di fondo, alla quale, in materia, Beccaria si affida: "Dunque la disciplina delle arti non deve essere coattiva e legislatrice, se non dove si prevegga che non mai o troppo tardi l'interesse privato giungerà ad unirsi col pubblico, e dove la scoperta delle frodi è lenta e remota, ed il guadagno che apportano è presente e considerabile". In tali casi "le leggi coattive, che impongono condizioni per le quali non si faccia tale cosa se non in tale maniera, e pene proporzionate ai contravventori, sono senza dubbio necessarie ed utilissime". In caso contrario "il complesso dell'arte intiera cadrebbe in discredito e svanirebbe una parte delle forze produttive di uno stato"..$^{143}$

Mi soffermo ancora sugli Elementi, toccandone qualche aspetto particolare. Alcune pagine importanti sono dedicate alla coltura e alla preservazione dei boschi, nonché alla disponibilità della legna "che quantunque non sia derrata d'alimento, lo è però di necessaria consumazione [...]". Il suo uso è necessario per i bisogni della vita, così come lo è per il "servizio quasi universale che rende a tutte le arti e manifatture $[\ldots]$ ". Sono sufficienti queste considerazioni "per conoscere quanto sia importante l'abbondanza e la facilità del commercio della legna". Nel determinare "i principi direttivi della economia de' boschi", quelli che si ispirano alla libertà sono preferibili e preferiti, ancora una volta, a quelli che "vengono suggeriti dal severo spirito di regolamento". Occorre pertanto non già iniziare con la proibizione di tagliare, con il divieto di estrarre, ma ben piuttosto, con rendere facili i trasporti, allargare le strade, rendere i canali agevolmente navigabili. "Bisogna dunque prima tentare quale effetto nasca dalla libertà avanti di intraprendere le rigorose precauzioni della schiavitù, rendere i boschi utili ai proprietari, sopprimere tutti i valori intermedi, e allora si vedrà facilmente abbondare una derrata così necessaria e così vantaggiosa”.

Ma la libertà nel suo provvido operare può incontrare ostacoli, siano essi i particolare rapporti dell'agricoltura con il commercio, gli elementi di diversa natura che possono negativamente incidere sull'interesse del proprietario alla conservazione del bosco, la difficoltà e la

143 BeCCARIA, Elementi [nt. 4], p. III, cap. IV, $₫ 32$, p. 330 s. 
lentezza della riproduzione del bosco stesso. Insomma, "la conservazione de' boschi può essere uno di quegli oggetti, che malgrado il sistema generale di un'assoluta libertà, può essere soggetto a qualche regolamento". ${ }^{144}$

E nel 1775 venne il tempo in cui parve opportuno sottoporre "la conservazione de' boschi [...] a qualche regolamento". Beccaria fu infatti chiamato ad esprimersi intorno ad una minuta di editto per il regolamento dei boschi, che dava attuazione ad una direttiva di Firmian ispirata a questa massima: "Le determinazioni però da prendersi non dovrebbero in massima restringere la libertà ad alcuno di far quell'uso che più troverà comodo, né indurne un sistema vincolante nemmeno riguardo i boschi”. Beccaria si trovò di fronte ad un progetto sul quale, secondo l'abituale coscienza dell'alta sua funzione - "il dovere della mia carica esige che io dica il mio parere con quella schiettezza che accompagnar deve i voti d'ogni ministro" -, espresse il proprio parere, che è chiarissimo fin dall'esordio: "Nella minuta s'adempie il proposito del positivo regolamento, ma si allontana troppo dall'altro intento che non sia troppo vincolante".

In effetti, è sufficiente scorrere il testo della minuta per rendersi conto della fondatezza di questo giudizio. Gli articoli di cui si compone si risolvono in una serie di vincoli, di divieti e di proibizioni muniti di severe sanzioni, delle quali pare opportuno proporre qualche esempio. Colui che, contro la volontà del padrone, avesse ardito di "far taglio alcuno sia di piante che di legne e di foglia", sarebbe incorso, oltre che nelle spese processuali, nella sanzione pecuniaria di cinque scudi da applicarsi per metà a vantaggio del denunciante "sì palese che segreto", e per metà a vantaggio del Fisco. Era inoltre prevista "la refezione e reintegrazione del danno al padrone del bosco" (art. 3). Per i danni prodotti dagli animali, la pena, a carico dei loro padroni, era di scudi cinque o di scudi uno, a seconda che si trattasse di bestia grossa o piccola (art. 4). Trovavano applicazione le "pene imposte dalle Nuove costituzioni di questo Stato al titolo De poenis $\mathbb{S}$ si quis incendium versiculus $S i$ vero id factum fuerit et versiculus $S i$ quis autem", nei confronti di chi avesse acceso un fuoco nei boschi "per consumare i ceppi e radici delle piante e ridurre in tal forma i detti siti ad altro uso, o per qualsivoglia altro effetto" (art. 5). ${ }^{145}$ Per

144 Ibid., p. II, cap. VI, \$\$ 67-69, pp. 224-229. 
tutti coloro che - "Deputati dell'Estimo, Sindici e Cancellieri" - risultassero complici nelle vendite, proibite alle Comunità, dei "loro boschi oltre il termine di un anno, e col patto così detto di grazia estensivo sino al termine di venti e più anni per terminarne il taglio" era comminata la pena di cinquanta scudi solidalmente, oltre la rimozione dall'ufficio e la nullità dei contratti (art. 11). La "pena di scudi dieci per volta $[\ldots]$ oltre la reintegrazione del danno ai proprietari, e anco maggiori secondo le circostanze dei casi [...]" colpiva chi avesse violato la proibizione di "levare la scorza alle querce ed altre piante $[\ldots]$ incidere le piante vive di larice, peccia pino ecc. per ricaverne la pece e la resina [...]" (art. 28). Da ultimo, ricordo ancora come a tutela delle "piante dei castagni fruttiferi", di fondamentale importanza sia per l'alimentazione degli abitanti del luogo sia per il profitto derivante dalla vendita dei frutti, a chi le tagliasse, per farne carbone o altro uso o, comunque, le danneggiasse, fosse comminata la pena di cinque scudi per pianta, "refezione del danno, ma eziandio [al]la carcerazione e altre maggiori secondo la circostanza dei casi" (art. 17).

Beccaria riflette. La modula di editto prevede troppe contravvenzioni che hanno il loro fondamento non nella malizia, ma nella negligenza o inavvertenza tali da dar luogo a liti e processi. Si configura un sistema che, anziché essere osservabile, sarebbe tale da esser pretesto di vessazioni e di lucro per i campari. Ed ecco l'alternativa, di per se stessa fonte di perplessità e di timore: o l'editto è osservato ed allora ne

145 Constitutiones Dominii Mediolanensis, decretis et senatus-consultis nunc primum illustratae curante comite GABRIELE VERRO [...], Mediolani 1747, liber IV de poenis, $\mathbb{S}$ Si quis incendium versicc. Si vero id, Si quis autem, p. 141. Le pene erano assai severe. Se il fuoco fosse stato acceso in luogo inabitato, la sanzione pecuniaria era quella del quadruplo del valore del danno procurato, danno la cui entità era determinata dalla dichiarazione giurata del soggetto passivo del reato, non disgiunta dalla previa stima giudiziale. Anche per questa pena ricorreva comunque la formula che d'ordinario corredava qualsivoglia risposta sanzionatoria prevista dal testo del 1541, testo fondamentale per la Lombardia spagnola ed austriaca: "et etiam alia poena graviori, usque ad mortem inclusive, arbitrio Principis, vel Senatus". Se poi l'incendio si fosse propagato "in alieno", questo è il testo della norma: [...] puniatur (ultra damnum, de quo stetur juramento damni passi, praevia taxatione ut supra) arbitraria poena etiam corporali, arbitrio Principis, vel Senatus, secundum facti qualitatem, per detentionemque procedi possit". 
consegue una massa di reclami e di sconcerti che scoraggiano gli abitanti della campagna con discapito dell'agricoltura; o non è osservato, "come è più probabile ad accadere, e questa legge correrebbe la sorte di tante altre che per essere state troppo minute e vincolanti sono andate in disuso ed in obblivione, con grave discapito della pubblica autorità e coll'avere solamente dato luogo a molte querele inutili".

Beccaria ragiona. Due sono oggi le cause che producono diminuzione dei boschi e delle piantagioni, considerato il disordine in quella generalità che conviene giacché gli inconvenienti particolari ben rare volte possono essere oggetto di legislazione: a) l'interesse degli abitatori della campagna alla distruzione dei boschi è per ridurli a miglior coltura, non per l'uso che ne fanno quando sono comunali; b) difficoltà nei trasporti. Per togliere la prima non è necessario, pensa e scrive Beccaria, la coattiva volta a proibire in qualsivoglia maniera e a qualsivoglia fine la distruzione dei boschi, coattiva moderata dalla previsione di possibili dispense da parte del Governo in casi particolari. Non molto vi è da sperare da una legge che ha frequente bisogno - e questo è il caso - di dispense. E poi una legge così cogente e vincolante scoraggerebbe l'agricoltore, l'affittuario, il possessore; renderebbe odiosa la proprietà dei boschi, che, anzi, dovrebbe esser resa pregevole e desiderabile; rivolgerebbe tutti gli sforzi a ottener dispense, a illudere la legge. "E' troppo noto ai lumi di questo Dicastero che tali coattive non possono essere dettate che dalla pubblica positiva necessità, ma non mai per conseguire una semplice utilità o vantaggio".

Beccaria rileva ancora: i boschi dei particolari vengono distrutti per ridurli a miglior coltura, poiché i possessori trovano utile ciò. Anche in questo caso l'interesse privato non può essere mortificato o abbattuto con leggi vincolanti e sanzioni punitive. La via da seguire è un'altra. La rendita dei boschi è minore di quella dei campi aratori. E i campi aratori nuovamente coltivati non pagano che per quella qualità nella quale sono stati stimati nel censo. Cioè: gli antichi campi aratori sono posti in prima squadra, i nuovi nelle ultime. Con maggior prodotto pagano meno di tributo. Occorre eliminare questo vantaggio. Toltolo, si toglierebbe il motivo di distruggere i boschi. Quindi niente coattive: Beccaria vorrebbe che i boschi esistenti fossero pur fatti a campo secondo la volontà dei possessori, ma che, resi campi, passassero immediatamente in prima squadra e pagassero il tributo corrispondente.

Quanto ai boschi comunali, sono distrutti perché gli utenti hanno 
grandissimo interesse alla loro depredazione. Hanno, di contro, un remoto e assai languido interesse alla conservazione di una proprietà, che è realmente di nessuno. La loro vendita ne renderebbe gelosa la custodia. Per quanto riguarda la seconda causa, consistente nella difficoltà dei trasporti, essa è "tolta col riattamento e conservazione delle strade, né solamente delle maestre e regie ma delle traverse e comunali, che come i vasi del corpo umano minimi e capillari, se sieno impediti ed imbarazzati, rendono difficile ed inferma la circolazione ne' vasi maggiori della vita". E poi, da ultimo, occorrerebbe aprire canali navigabili. ${ }^{146}$ Pare davvero di leggere le parole che Beccaria ebbe a scrivere negli Elementi. ${ }^{147}$

146 BeCCARIA, Atti di Governo, VI [nt. 25], doc. 380, Regolamento dei boschi (relazione [1775]), pp. 656-670. Beccaria avrà occasione di ritornare frequentemente ed in modo importante sulla materia dei boschi [Capra, 1984, pp. 483-485], (oltre ai documenti citati in nota 139 e qui, di seguito, v., per esempio, BeCCARIA, Atti di Governo, VII [nt. 139], doc. 743, 28 settembre 1782, p. 698; doc. 877, Proposizioni fatte dal Marchese Consigliere Beccaria nell'atto della relazione (appunti su verbale, 10 dicembre 1783), pp. 872-883; BECCARIA, Atti di Governo, VIII [not. 19], doc. 891, 23 aprile 1784, pp. 35-39; doc. 1192, 27 luglio 1785, p. 458 s.). Sono documenti nei quali, dato lo stretto rapporto che lega la materia dei boschi con quella delle miniere e delle fucine (non potendo ora illustrare questo legame nei suoi vari ed importanti risvolti, mi limito a riportare alcune parole che si leggono in una relazione di Beccaria: "Per eseguire nella miglior maniera la delegazione di cui Sua Altezza Reale mi ha onorato relativamente al miglioramento dei travagli delle miniere di ferro ed alla sussistenza dei medesimi, dipendenti dalla ristorazione e conservazione dei boschi [...] La sussistenza dei travagli delle miniere dipende onninamente dai boschi, dei quali perciò Sua Maestà comanda la ristorazione ed aumentazione nei siti vicini alle medesime" (BECCARIA, Atti di Governo, VII [nt. 139], doc. 764, 29 gennaio 1783, pp. 726-737, in particolare, pp. 726, 730), ed a ricordare che l'anno successivo si ordinava che nelle vendite e nei livelli dei boschi comunali, come pure nelle vendite del loro taglio periodico, venissero preferiti "i proprietari delle miniere di ferro o dei forni in cui esse si fondono come pure i proprietari delle fucine grosse, in cui si affina o purga il ferro prodotto dai forni [...]" (BECCARIA, Atti di Governo, VIII [not. 19], doc. 885, 17 marzo 1784, p. 25) [Frumento, 1963, p. 359-366]), ritroviamo all'opera persone che, impegnate nel settore, già ci sono note, quali Francesco Mornico e padre Ermenegildo Pini (cfr. retro pp. 305-307), il quale, sempre fortemente stimato da Beccaria, nel 1782 era stato "destinato superiormente a soprintendere colla singolare sua perizia ai lavori delle miniere ed anche alla miglioria de' boschi inservienti al buon travaglio delle stesse [...]" (BECCARIA, Atti di Governo, VII [nt. 139], doc. 747, 2 dicembre 1782, pp. 702-704, sul punto, p. 702).

147 V. retro p. $351 \mathrm{~s}$. 
Circa dieci anni più tardi, nel 1787, Beccaria avrebbe avuto modo di ribadire la sua fiducia nel libero commercio della legna: "[...] finalmente atteso l'esempio di Cremona, Lodi e Pavia, che non ha calmiere in questo genere, non si possa abolire la meta anche a Milano, e quali ne sarebbero le conseguenze, in confronto dei vantaggi da sperarsi per la maggior affluenza de' venditori e per il minor incentivo alle frodi introdotte o per l'avidità del guadagno malgrado il rigore della meta, o per rifarsi della medesima quando non sia veramente proporzionata al primo costo". ${ }^{148}$

Eccoci ad un altro Piano, quello per la caccia, al quale già si è avuto occasione di fare riferimento. Si tratta di una materia -"materia che", scrive Beccaria, "ha molto interessanti rapporti con tutto il restante sistema di publica amministrazione" ${ }^{149}$ - nella quale particolarmente intenso è il rapporto tra diritto penale ed economia, non solo per la sua natura e sostanza intrinseche, ma anche sotto il profilo della sistematica penalistica. Mi spiego. Ci mette sull'avviso una comunicazione della Corte al III Dipartimento del Consiglio di Governo, che suona così: "Sollecita [la Corte] la revisione dei vecchi editti in materia di caccia, confermando che alla potestà politica dovrà essere attribuita la giurisdizione primitiva". Il riscontro è il seguente: "Raccolte finalmente le opportune notizie, si sta attualmente estendendo il Piano del nuovo regolamento delle Cacce, che a suo tempo sarà rassegnato". Ma chiaro, quanto mai chiaro è Beccaria al riguardo: "Ora [...] che sono giunte e da porsi in corso le leggi punitive tanto criminali che politiche [...] in virtù delle quali non si può più dubitare che, la caccia essendo un ogetto meramente politico, anche le trasgressioni alle leggi della medesima non siano punibili con quelle regole e da quei tribunali che a simili oggetti sono competenti $[\ldots]$ "... ${ }^{150}$

La competenza, che nel penale viene attribuita alla "potestà politica", deriva dalla distinzione introdotta dal Codice penale di Giuseppe II tra delitti politici e delitti criminali. La repressione dei delitti politici era disciplinata nella seconda parte del codice penale del 1787, che comprendeva oltre ad una nutrita serie di infrazioni di polizia, anche

148 Atti di Governo, IX [nt. 19], doc. 1590, 5 febbraio 1787, p. 92 s.

149 BeCCARIA, Atti di Governo, XI [nt. 9], doc. 2851, 19 gennaio 1789, p. 37.

150 BeCCARIA, Atti di Governo, X [nt. 37], doc. 2206, 21 gennaio 1788, p. 50 s.; BeCCARIA, Atti di Governo, XI [nt. 9], doc. 2851, 19 gennaio 1789, p. 36 s. 
delitti come la bestemmia, l'eresia, l'adulterio, la sodomia e i furti minori. La normativa ivi accolta disorientava non solo per il tipo di risposta sanzionatoria, per lo stravolgimento di schemi tradizionali e consolidati, ma anche perché creava una pericolosa dipendenza delle magistrature di prima istanza (podestà e pretori locali) dalle Intendenze politiche. In altre parole i delitti politici erano giudicati, sì, dai magistrati ordinari di prima istanza, come ho appena detto, ma solo in quanto essi operavano come delegati del Governo, dal quale ricevevano la loro autorità e da cui dipendevano.

In realtà il quadro ora delineato ha come riferimento normativo, non già la Giuseppina, che mai entrò in vigore in Lombardia, ma - oltre alla Norma interinale del processo criminale [Provin, 1990; Pagano, 2011, pp. 172-176], che si lega al nome di Karl Anton von Martini [Capra, 1985; Cassi, 1990, pp. 362-369] e alle norme che disciplinavano le competenze e le funzioni delle Intendenze politiche [Mozzarelli, 1990, pp. 89-108] - le Instruzioni per li magistrati politici sul modo dell'inquisizione, condanna ed esecuzione contro i rei di delitto politico $(30$ aprile 1787), cui seguirono il Piano giurisdizionale per la cognizione e punizione de' delitti politici, nonché la Norma per la procedura da osservarsi ne' delitti politici (11 ottobre 1787). Un complesso, quest'ultimo, di norme procedurali provvisorie che, approvate da Giuseppe II, dovevano, o avrebbero dovuto garantire dal pericolo di vessazioni i cittadini indiziati, circoscrivendo l'ambito di competenza della Polizia entro ristretti confini: "Occorre qui ripetere la nozione preliminare, che l'Officio di Polizia non ha ingerenza alcuna nella procedura inquisitoriale, o nella condanna neppure ne' casi di delitti politici, o di Polizia, fuorché l'arresto dei delinquenti già sufficientemente indiziati per tali, e dopo quello seguito deve consegnare i detenuti alla custodia, o alla carcere del Giudice competente [...]" [Brambilla, 2003, p. 106 nota 89]. La cognizione e la punizione dei delitti politici dovrebbe appartenere "à rispettivi podestà e pretori locali dipendentemente dal Dicastero politico ch'è il Governo, e ne' casi criminali rispettivamente de due tribunali d'appellazione [di Milano e di Mantova]".

Non sto ora a dire del ricordato dispaccio dell'11 ottobre 1787 , che innovava, attribuendo la giurisdizione politica nelle città alle Congregazioni municipali (fuori di esse, nelle campagne, rimaneva ferma la giurisdizione delle preture, dipendenti, come del resto le stesse Congregazioni, dall'Esecutivo). Era un sistema di norme dettato dall'amor di perfezione (negativo, ormai lo sappiamo, per Beccaria). 
Giuseppe II l'aveva programmato sul presupposto teorico di una combinazione, non già di una confusione delle funzioni. Kaunitz si rendeva conto delle difficoltà che l'innovazione comportava: "La divisione dei delitti in criminali e politici tutta nuova e ignota in passato a' giudici, particolarmente d'Italia [...] rende necessario di camminare nella procedura giudiziaria con molta delicatezza e circospezione per non urtare e collidere la podestà politica con i Tribunali di Giustizia" ${ }^{151}$ Collisioni in effetti si verificarono, nelle città di provincia, tra le due autorità locali, l'intendente politico e il pretore.

Disorientamento ed opposizioni ben presto si manifestarono anche nell'opinione pubblica. Pietro Verri, tra altri: "Si creò una capricciosa divisione ne' delitti, formando una classe di delitti politici, e con

151 Le Instruzioni, unitamente al post scriptum di Kaunitz 30 aprile 1787 dal quale sono tratte le parole trascritte anche nella pagina che precede, sono in ASM, Giustizia punitiva, p.a., cart. 1, fasc. 2; il Piano giurisdizionale e la Norma per la Procedura sono in ASM, Dispacci reali, cart. 266; Giustizia punitiva, p.a., cart. 3. [Garlati, 2006, p. 502 ss., con squarci di law in action di grande interesse]. In realtà le paventate collisioni sarebbero state scongiurate solo dall'effettiva chiarificazione del complicato, se non confuso, quadro delle competenze in materia di delitti politici, dalla risoluzione dei conflitti di competenza a loro volta subordinati all'adattamento e poi all'introduzione del Codice giuseppino in Lombardia. Ma i lavori della Giunta istituita da Giuseppe II (dispaccio 3 ottobre 1787, cui sono unite le Instruzioni per l'adattamento da farsi del Codice Generale austriaco sopra i delitti e le pene, in ASM, Dispacci reali, cart. 266; Giustizia punitiva, p.a., cart. 3) non pervennero al divisato obiettivo. Pertanto, "Il mancato adattamento del Codice Penale austriaco, il successivo abbandono della bipartizione fra delitti politici e criminali [20 gennaio 1791], l'abolizione della Polizia governativa e delle Intendenze Politiche [30 gennaio 1791] avrebbero però fatto naufragare ogni tentativo innovatore" [Provin, 1990, p. 47. Ivi, pp. 121-125, sono pubblicati gli Appuntamenti presi nella Conferenza 17 luglio 1786 sopra $i$ seguenti quesiti. Si tratta di un documento prezioso in quanto, posto a cavaliere tra l'editto 11 febbraio 1786 e l'entrata in vigore della Norma interinale, verbalizza una seduta del Consiglio di Governo alla quale prese parte anche il Martini. La risposta al quesito "Decimoquarto. Quale sarà il confine fra l'autorità della Polizia e la Criminale?" è così formulata: "Si è detto che spetteranno alla Polizia tutte le contravvenzioni agli Editti veglianti in materia di Polizia, e Governativa; ed ove si tratterà di prevenire i delitti, mediante la detenzione de' sospetti, vagabondi, validi mendicanti. Dovendo poi consegnarsi alla Giustizia Criminale li detenuti, contro i quali emergeranno titoli di Criminalità. Che poi a suo tempo si devolveranno pure alla Polizia i delitti minori, che nel nuovo Codice Austriaco saranno esclusi dalla categoria dei delitti Criminali, e dovranno punirsi in via sommaria, ed economica: e che frattanto si riterrà ciò, ch'è stato appuntato al num ${ }^{\circ}$ quarto circa li delitti riferibili, o non riferibili”, ibid., p. 125]. 
questo vocabolo si stabilì che il Capo del Consiglio Governativo senza formalità di giudizio, di solo suo ordine, potesse condannare anche a pene afflittive e disonoranti senza altra difesa o processo". ${ }^{152} \mathrm{E}$ Beccaria? Il Beccaria chiamato ad esprimere il suo giudizio sul codice penale, giuseppino appare, come dire, più timido rispetto a quello di trent'anni prima. Innanzitutto si allontana dal principio del diritto penale a soggetto unico abbracciato nel Dei delitti e delle pene: "A chi dicesse che la medesima pena data al nobile ed al plebeo non è realmente la stessa per la diversità dell'educazione, per l'infamia che spandesi su di un'illustre famiglia, risponderei che la sensibilità del reo non è la misura delle pene, ma il pubblico danno, tanto maggiore quanto è fatto da chi è più favorito [...]". Ovvero, in modo più secco: "Io mi ristringerò alle sole pene dovute a questo rango, asserendo che esser debbono le medesime pel primo e per l'ultimo cittadino". ${ }^{153}$ Ma da questi principi, per l'appunto, si allontana: "[...] nei delitti politici $[\ldots]$ si deve avere moltissimo riguardo alla condizione delle persone, perché il bastone che può correggere un facchino, avvilisce ed annienta un nobi-

152 P. Verri, Pensieri del Conte Pietro Verri sullo stato politico del Milanese nel 1790, in ID., Scritti politici della maturità, a cura di C. CAPRA, vol. VI dell'Edizione Nazionale delle Opere, Roma-Milano 2010, IV Idee politiche del Conte Pietro Verri da non pubblicarsi (1763?-1790), pp. 390-425, sul punto, p. 402. Similmente già si era espresso Beccaria. Nel rivolgersi a Wilczeck, capo del Consiglio di Governo dal 1786, lo invitava a riflettere che, se era vero che nei delitti politici la sorte del reo, quanto all'inflizione delle pene del bastone, della berlina e del lavoro pubblico, pareva dipendere "da un corpo collegiale", era altresì vero che la collegialità, "paragonando la maniera con cui [essa] è costituita nel Consiglio di Governo, e l'influenza del voto dei singoli individui con quella dei Tribunali d'apellazione" competenti per i delitti criminali, era soltanto apparente. La conseguenza? "[...] che i rei politici sono in peggior condizione dei rei criminosi”. Beccaria si avvede che queste considerazioni potevano essere intese come volte a colpire e scalfire l'autorità di Wilczeck e prontamente scrive: "Vostra Eccellenza è troppo grande di animo per imputarmi l'ardire di una tale riflessione, la quale non è certamente applicabile al caso presente, ma può venire nel futuro la circostanza che ne faccia vedere l'inconveniente, e l'Eccellenza Vostra sa meglio di me che le leggi e i codici devono esser fatti per la diuturnità dei tempi, e non per le persone che attualmente hanno in mano la pubblica autorità" (BECCARIA, Atti di Governo, IX [nt. 19], doc. 1846, Codice generale (relazione, [giugno 1787]) Brevi riflessioni [nt. 124], p. 489 s.).

153 BeCCARIA, Dei delitti e delle pene [nt. 6], $\$$ XXI Pene dei nobili, p. 74 s. [Burgio, 2000, p. 95 s.]. 
le, un onesto negoziante e qualunque civile persona, e involge tutta la di loro famiglia nella più luttuosa ignominia [...]". In buona sostanza, Beccaria, mentre rimane fedele alla vecchia tesi egualitaria per quanto riguarda i delitti criminali, "nei delitti politici, che non suppongono malizia, $[\ldots]$ e che non tendono direttamente a distruggere la società né offendono il diritto naturale, che sono mere colpe e non doli [...]" lo status personale riemerge nella sua rilevanza [Sbriccoli, 1997, p. 180 nota 4]. ${ }^{154}$

Pochi mesi dopo aver manifestato queste opinioni nelle Brevi riflessioni elaborate intorno al Codice penale giuseppino, Beccaria fu chiamato a pronunciarsi in merito alla pena da infliggere a sei macellai contravventori, rei di "uccisione di bestie cavalline" e della vendita vietata delle loro carni. Esclusa, in disaccordo con il voto del Fisco, l'applicabilità della pena della frusta, in quanto non ricompresa tra le pene politiche, la scelta era limitata tra quelle del bastone, della berlina e dei pubblici lavori (c.p., p. II, cap. II, $\$ 10$ ). Si trattava di pene afflittive e infamanti e, in quanto tali, non idonee a conseguire lo scopo al quale avrebbero dovuto tendere le pene politiche: la correzione del reo, in primo luogo e, di poi l'esempio. Pertanto esse "devono essere commisurate in modo che l'esempio non nuocia alla correzione, che è il loro scopo principale, e perciò devono essere di qualità molto diversa dalle prime [le pene criminali], di gran lunga più miti, meno durevoli e, per quanto si può, niente infamanti, giacché, prodotta l'infamia, svanisce ogni speranza di correzione, che si vuole e che si deve esigere dalle pene politiche". Se questo era l'auspicio del Beccaria delle Brevi riflessioni, la realtà imponeva ora la scelta tra le pene indicate, che, "per essere annoverate fra le pene politiche, possono dalla potestà economica senza pregiudizio della facoltà giuridica erogarsi $[\ldots]$ ". Esclusa dunque la frusta, la preferenza espressa da Beccaria cade sulla pena della berlina, non già su quella dei lavori pubblici in ferri, in quanto essa "è una pena assai più esemplare per quelli che fossero tentati di commettere simili frodi" e capace, inoltre, di soddisfare "quel pubblico che ne è stato la vittima e che si aspetta di vedere la punizione di questi rei". La pena dei lavori pubblici, da scontare nell'Ergastolo di Pizzighettone, così come il carcere annuale con inasprimenti, "sottraendo i delinquenti dagli occhi del pubblico, non lascia di

154 BeCCARIA, Atti di Governo, IX [nt. 19], doc. 1846, Codice generale (relazione, [giugno 1787]) Brevi riflessioni [nt. 124], p. 483. 
essere egualmente infamante, ma di gran lunga meno esemplare e meno notoria”.

Non potendo indugiare sulle pene, e sulla loro quantificazione, indicate da Beccaria come giuste e necessarie per ognuno dei sei contravventori, basti qui offrire il quadro sanzionatorio che, in generale, risulta dalla relazione beccariana: esposizione alla berlina per una sola volta - ovvero "doppia" o anche triplicata, "[...] da infliggersi nella distanza di otto giorni dall'una all'altra esposizione, ritenuto [il reo] nell'intervallo in carcere stretta con ferri ai piedi" - nell'ora in cui maggiore fosse il concorso del popolo. Il condannato doveva inoltre portare al collo un cartello esprimente il titolo del delitto commesso. Al momento della dimissione, al reo doveva essere intimato il precetto "d'astenersi in avvenire da simili contravvenzioni" sotto comminatoria, in caso di violazione del precetto stesso, dell'inflizione di una pena che poteva consistere nella duplicata esposizione alla berlina, nell' "immediata prigionia", nei pubblici lavori "e anche maggiore secondo le circostanze". Rispetto al Beccaria del Dei delitti e delle pene, rispetto al Beccaria teorico, che dire? L'esecuzione delle pene in pubblico, nel tempo in cui più alta fosse l'affluenza del popolo, l'apposizione del cartello al collo del condannato, in forme, dunque altamente spettacolari sotto questo profilo, nulla di nuovo rispetto alla prassi senatoria, nelle cui sentenze si rinvengono con martellante frequenza le formule "hora frequentioris populi concursus", ovvero "in die mercatus" (Massetto, 1994, p. 359 ss.) - rispondevano indubbiamente al fine di distogliere il reo ed i consociati dal commettere nuovi illeciti, con soddisfazione del principio della prevenzione speciale e generale. Era raggiunto "il fine politico della pena", identificato con "il terrore degli altri uomini"[Cattaneo, 1997, p. 84]; "Il fine [della pena] dunque non è altro che d'impedire al reo dal far nuovi danni ai suoi cittadini e di rimuovere gli altri dal farne eguali”. Gli inasprimenti previsti (carcere stretta, ferri ai piedi) sembrano in contrasto con il principio, pur strenuamente difeso, della mitezza della pena, mentre quello della certezza pare contraddetto dalla formula ricorrente "ed altra [pena] anche maggiore secondo le circostanze". E' ben vero che Beccaria, rispetto al voto del Fisco, non ricorre alla locuzione "maggiore od arbitraria", ma è altresì vero che il riferimento alla "qualità delle circostanze", che, non specificate, sono lasciate alla discrezionale valutazione del magistrato, apre le porte all'arbitrio, a quell'arbitrio così aborrito da Beccaria in quanto "distruttore" del dogma della certezza del diritto e, pertanto, in 
assoluta opposizione con "leggi ed ordini altrettanto semplici che universali". 155

Ma poniamo ora mente a Beccaria alle prese con il Piano della caccia, tenendo presente che il Codice penale giuseppino annoverava il cosiddetto delitto di caccia tra quelli politici, ${ }^{156}$ con la previsione di pene corporali, escluse le pecuniarie. Beccaria mette subito le carte in tavola. Gli pareva necessario distinguere nelle contravvenzioni di caccia due classi principali di delitti, "gli uni sono in danno della regalìa semplicemente, gli altri sono qualificati da circostanze di sua natura aggravanti e più direttamente opposte al buon ordine pubblico". Tra i primi rientrano l'andare a caccia senza licenza, l'andare nei luoghi riservati, l'andarvi nei tempi proibiti. Realizzano frode alla regalìa e quindi devono essere puniti con multe, le quali, anche nell'attuale legislazione, sono in vigore per tutti i frodatori delle altre regalie come, ad es., sale e tabacco. Tra i secondi rientrano "l'opposizione minacciosa e violenta a' campari, l'entrare ne' seminati senza riguardo all'altrui proprietà e simili, che sono di sua natura opposte al buon ordine della società e contengono una maggior malizia di quellocché sia la semplice defraudazione della regalia”. In quanto tali, sono punibili con pena corporale, con privazione della libertà e con qualche inasprimento a misura delle circostanze qualificanti il delitto e secondo la qualità delle persone. Questo è il criterio di fondo, che Beccaria esprime: "e con il quale andrò, dove occorre, divisando la qualità della penale conveniente alle rispettive

155 Per Beccaria teorico, cfr. BECCARIA, Atti di Governo, IX [nt. 19], doc. 1846, Codice generale (relazione, [giugno 1787]) Brevi riflessioni [nt. 124], p. 479; BECCARIA, Dei delitti e delle pene [nt. 6], $\$ \$ S$ XVI Della tortura, p. 63; XII Fine delle pene, p. 55; BeCCARIA, Prolusione [nt. 5], p. 188 [Di Renzo Villata, 2000, p. 30]. Per Beccaria pratico, v. Atti di Governo, IX [nt. 19], doc. 2006 (relazione [settembre 1787]), pp. 688-692.

156 Allgemeines Gesetz über Verbrechen und derselben Bestrafung-Codice generale sopra i delitti e le pene, Vienna-Rovereto 1787, parte seconda De' delitti politici e delle pene politiche, cap. IV De' delitti Politici, i quali feriscono la facoltà, e $i$ diritti de' Concittadini, $\$ 30$, il cui punto $b$ contemplava il "furto di animali selvatici intrapreso in qualunque maniera da persona non autorizzata alla caccia sia anche su i propri fondi". Quanto al regime sanzionatorio il $\$ 32$ prevedeva: "La pena del furto come delitto Politico è prigionia, castigo di bastonate, e di altri inasprimenti, e ciò a misura del maggiore, o minor dolo intervenutovi, e del danno recatone al derubato. In caso di ricadute si dovrà prendere provvedimento, affinché il reo anche dopo la sostenuta pena sia tenuto di vista per qualche tempo dalla Polizia ad effetto di tenerlo, mediante regolare condotta, sulla strada di procacciarsi onoratamente il suo sostentamento". 
contravvenzioni, procurando di stabilire, dove convengono, i limiti dentro de' quali il giudice politico dovrà contenersi". ${ }^{157}$ E' da ricordare che il principio nel quale Beccaria fermamente crede, nella determinazione e quantificazione delle pene, è quello che della moderazione e dolcezza. L'esperienza gli aveva dimostrato l'inutilità di percorrere vie diverse da quella indicata: "La severità istessa delle pene comminate ai contraventori dal vigente regolamento $[\ldots]$ ha prodotto l'effetto dell'inosservanza, come suole accadere ogni volta che ai delitti solamente politici e non propriamente criminosi s'impongono pene troppo forti, resistendovi la naturale commiserazione, dal che ne è venuto che tutte le contravenzioni o giacevano impunite o si risolvevano in composizioni delle quali probabilmente il maggior partecipante non era la Camera, che soffriva il danno della contravenzione stessa" ${ }^{158}$

A proposito di un nuovo editto di Riforma dei pesi e delle misure, Beccaria non si allontana dall'indicato principio: "le penali del quale [editto] dovrebbero essere dolci dove si tratti di pura negligenza, più severe dove si tratti di espressa frode; dovrebbe avere poche leggi e poco vincolanti il pubblico, ma si dovrebbe tenere man forte all'osservanza di quelle, parendomi che, colla sola inalterabile fermezza, si possa sperare buon esito dalla voluta riduzione". ${ }^{159}$ Torno a ripetere, Beccaria per delitto criminale intendeva "quello di cui l'indole è tale che tende direttamente alla distruzione del vincolo sociale, quando non fosse punito e represso [...]". Per delitto politico intendeva "quella trasgressione o colpa che, rendendo imperfetta la società, non tende che indirettamente alla di lei distruzione". Che per i primi, "a caratterizzarli non v'è bisogno di leggi positive, poiché per tali sono qualificati dal diritto della natura e delle genti", mentre i secondi "prendono la principale loro qualificazione dalle leggi positive [...]". Occorreva sulla base di questi principi fissare pene corrispondenti e proporzionate all'“indole" dell'illecito. ${ }^{160}$

Il cap. 33 del Piano della caccia prevedeva la fattispecie consisten-

157 BeCCARIA, Atti di Governo, XI [nt. 9], doc. 2851, 19 gennaio 1789, p. 52 s.

158 BeCCARIA, Atti di Governo, IX [nt. 19], doc. 1689, Attività del Dipartimento III nel 1786 (relazione, 31 marzo 1787), pp. 223-267, sul punto, p. 265.

159 BECCARIA, Atti di Governo, VII [nt. 139], doc. 547, 25 gennaio 1780, p. 274 s.

160 BeCCARIA, Atti di Governo, IX [nt. 19], doc. 1846, Codice generale (relazione, [giugno 1787]) Brevi riflessioni [nt. 124], p. 478 s. 
te nel non rispettare i campi seminati. Se condivisibile era la previsione di siffatta fattispecie, non altrettanto lo era la risposta sanzionatoria: 300 scudi d'oro, risarcimento del danno, pena della galera in caso d'impotenza (si ripeteva l'antica massima "Qui non habet in aere luat in corpore"). "La pena prevista mi pare incongrua ed eccessiva e, per conseguenza non eseguita, non eseguibile [...]. Dico incongrua perché l'entrare ne' seminati altrui, calpestando e malmenando l'altrui proprietà, senza riguardo al danno che si può recare -si ricordi che Beccaria, in ciò non poco criticato, sosteneva che la vera misura del reato è il danno procurato alla società- non è una frode della regalìa, ma un atto colpevole che $[\ldots]$ può meritare una punizione ma non pecuniaria, bensì personale $[\ldots]$; per istabilire una pena proporzionata e corrispondente all'indole del delitto, conviene distinguere il cacciatore licenziato dal non licenziato invadente i seminati altrui, e parimenti distinguere due contravvenzioni diverse per punirle diversamente. Il primo, che senza licenza va a caccia, deve punirsi colla stabilita pena pecuniaria, e quando sia colto, e risulti cacciatore, ne' seminati, a istanza del danneggiato, oltre il rifacimento del danno, da giudicarsi da chi sarà di ragione in via civile, debba anche assoggettarsi alla pena politica di carcere o di arresto breve da una settimana fino ad un mese, ed anche di un mese fino ad un anno se sianvi concorsa violenza o minaccia, e colla stessa pena debba punirsi l'invasore de' seminati, ancorché sia cacciatore licenziato, nel qual caso non dovrà subire la pena pecuniaria, ma resterà privato della licenza" ${ }^{161}$

Un'altra fattispecie (cap. 35) proibisce "le cacce generali, ossia a restelli, le tagliole, fossi o lovare per prendere quadrupedi", comminando la pena di 100 scudi. Beccaria, espresso l'auspicio che la norma fosse redatta in termini più precisi ("Conviene definire che sia caccia generale, o caccia a restello, e determinare il numero dei cacciatori abbinati che si richiede a caratterizzare una di queste caccie [...]"), riteneva che la penale di 100 scudi fosse troppo rigorosa e che potesse "bastare la doppia penale di venti scudi ed il doppio onorario di quattro scudi a' campari da pagarsi da ciascun contravventore”. Mitezza della pena, dunque, che però si attagliava solamente a chi contravvenisse al divieto relativo alle "caccie generali", non già per chi ponesse tagliole o facesse fosse: questi mezzi "di far caccia sono tali che non solo

161 BeCCARIA, Atti di Governo, XI [nt. 9], doc. 2851, 19 gennaio 1789, p. 61. 
mettono in grave pericolo il bestiame domestico, ma le persone eziandio e chi, per avidità di cacciagione [...] disprezza non solo il danno ma anche il pericolo personale de' suoi simili, è assai più colpevole e la pena proporzionata dev'essere corporale, oltre la solita pena come contravventore alla caccia e il rifacimento del danno" ${ }^{162}$

La pena dunque deve essere congrua, proporzionata alla gravità dell'illecito, ma, in pari tempo deve essere inevitabile, inesorabile. Si tratta di principi applicati nelle consulte, sulle quali si è finora soffermata l'attenzione, principi che vengono limpidamente riassunti in quell' 'aurea' frase espressa dalla corrente, che risultò minoritaria in seno alla Commissione leopoldina istituita il 24 gennaio 1792: “[...] non tanto la gravità della pena ma l'inevitabilità di quella, purché sia proporzionata ai delitti, è il mezzo più efficace per reprimerli $[\ldots]$ ". ${ }^{163}$ Nella consulta al Piano della caccia ancora si legge: "Ciò che poi, in compenso della pena più mite, dovrebbe stabilirsi per massima costante si è di escludere affatto le composizioni. Quando le pene sono gravi e severe contro delitti di questa natura, che appena si possono chiamar tali [proibizione di andare a caccia senza licenza], ma piuttosto coi più miti nomi di contravvenzioni, colpe ecc., nasce la necessità delle composizioni, reclamando altamente contro l'asprezza delle leggi positive, il sentimento naturale di equità e di commiserazione. Ma dove la legge è di sua natura mite, devono essere inesorabili gli esecutori di essa, perché la legge non cada in disprezzo, e non si apra la porta agli abusi ed alle facili collusioni”" ${ }^{164}$

L'inesorabile applicazione della pena comporta quindi la netta avversione per le composizioni, delle quali un cenno già si è fatto in precedenza, composizioni "sempre dannose all'esatta osservanza delle leggi, massimamente quando sono, come dovrebbono essere sempre, dolci e moderate $[\ldots .$.$] . La composizione dev'essere riservata alla supe-$ riore autorità del Consiglio, per qualche singolare circostanza, che

162 Ibid., p. 63, ove Beccaria così conclude: "Tale pena dovrebbe essere la carcere o l'arresto fino ad un mese, secondo la qualità delle persone, quando la tagliola, fossa o lovara sia nella campagna, perché le poste ne' luoghi frequentati e strade pubbliche sono espressamente soggette alla sanzione politica del Codice penale”.

163 BeCCARIA, Atti di Governo, XVI-II Appendice Voto degli infrascritti individui [...] [nt. 18], p. 969.

164 BeCCARIA, Atti di Governo, XI [nt. 9], doc. 2851, 19 gennaio 1789, p. 59. 
possa meritare il sempre pericoloso deviamento dalla regola generale" ${ }^{165}$ Del resto, si legge ancora nella consulta sul Piano della caccia: "Il piccolissimo prodotto di queste composizioni [...] mentre pochissime o nissune sono le penali intiere che si sono ritratte, fanno vedere, nel caso concreto, quanto sia opportuna l'applicazione alla caccia della massima generale di escludere le composizioni". ${ }^{166} \mathrm{E}$ ancora: "il sopprimere o il comporre arbitrariamente le contravvenzioni è una colpa, che dicesi assai frequente ne' campari e simil sorta di gente, e la penale dev'essere anche corporale oltre il rifacimento della multa, ma, questa, circoscritta dentro certi limiti, come di un mese fino ad un anno, secondo le circostanze più o meno aggravanti, e non maggiore ad arbitrio, come esprime la grida" ${ }^{167}$

I campari, che già abbiamo conosciuto, svolgevano una funzione, sulla carta, assai importante e benefica, ma, nella prassi, erano poco, pochissimo o addirittura per niente apprezzati. In numero di 45 - ovvero di $50,{ }^{168}$ - con il salario annuo di lire 84 , cui si aggiungeva qualche altra assai misera provvidenza, avevano fondamentalmente il dovere di "perlustrare ex officio la rispettiva loro provincia, massimamente ne' siti più esposti alle frodi e più abbondanti di caccia" e di "levare le arme, le reti e tutto ciò che riguarda la caccia alli contravventori trovandoli in fragranti non muniti di licenza, senza però usare atti o parole ingiuriose" ${ }^{169}$ Intorno alla personalità dei campari, sono queste le riflessioni di Beccaria: "Le persone dedite al vino e alla crapula, quelle che si sono rese sospette di delitti o che ne siano realmente stati puniti hanno delle qualità certamente esclusive". Nemmeno gli sbirri possono aspirare ad essere campari, né "coloro che per effetto dell'opinione generale sono communemente risguardati con qualche ribrezzo dal commune degli uomini [...]". E poi, dal momento che chi contravviene alla legge "non commette un'azione per sé indecente, così quelli che devono invigilare all'osservanza di quelle devono essere persone che, ancorché poste nella più bassa sfera, in essa godano di qualche credito di probità e di saviezza. In questa maniera, la qualità delle persone accrediterà l'ufficio e l'uf-

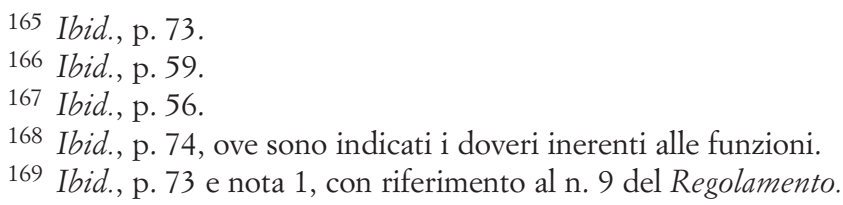


ficio sarà considerato ed ambito dalle persone. Soprattutto crederei che non potesse aspirare all'ufficio di camparo se non chi sapesse leggere e scrivere. Io ho osservato fra i contadini, che generalmente sono i migliori, que' pochi che nella commune ignoranza si distinguono con questo primo grado di coltura, e l'esiggerlo come condizione irrefragabile a poter essere campari, può animare sempre più le rustiche persone ad acquistare questi primi rudimenti delle umane cognizioni [...]". Si tratta di una qualità che si rende necessaria dal momento che i campari devono stendere le denunce, "le quali è bene che siano fatte in iscritto o almeno firmate dal denunciante". ${ }^{170}$

Nei confronti dei cacciatori, i campari dovranno tenere un moderato contegno. Tanto più che "le persone che vanno a caccia sono persone o nobili o assai civili e, quando non lo fossero, sono uomini, sono sudditi e, ancorché contravventori alle leggi di società, non lo sono di quelle di natura che ha incominciato col farci cacciatori, né la vigilanza sulla caccia deve risolversi in guerre ed ostilità". I campari non dovevano mai essere in numero minore di due, ovvero un camparo accompagnato almeno da un testimone: il camparo da solo non può compiere nessuno degli atti che pur gli competono, neppure chiedere l'esibizione della licenza. In ogni caso, costoro potranno e dovranno operare, "ma senza violenza, senza mettere le mani adosso a nessuno, senza minacciar con l'armi, che non devono portare che per necessaria difesa" ${ }^{171}$ Per converso, ove il contravventore rifiuti di fare ciò che deve, la deposizione del secondo camparo o del testimone che concordi con l'assunto del camparo, "basterà a fare piena fede nel giudizio politico della contravvenzione e in tal caso il rifiuto semplice dovrà essere punito con doppia multa. Nel caso il rifiuto si manifesti con opposizione violenta o minacciosa [...] la contravvenzione cangia di natura e deve considerarsi per un vero delitto il quale deve punirsi come politico o criminoso, secondo che sarà stabilito nel Codice dei delitti e delle pene" ${ }^{172} \mathrm{E}$ con questo, abbandono il Piano per la caccia e la relativa consulta beccariana, se non per ricordare che il nuovo regolamento doveva avere due obiettivi di fondo, che si risolvevano in altrettante massime: conservare la regalia e, in piena osservanza di quella "moderazione e dolcezza delle

\footnotetext{
170 Ibid., p. 70 s.

171 Ibid., p. 71 s.

172 Ibid., p. 72.
} 
pene, stata più volte espressamente inculcata dalle osservazioni della Regia Imperial Corte, e coerente alle massime del nostro clementissimo Sovrano, onde angustiare il meno che si può la libertà civile in una azione per se stessa innocente e solamente reprimibile per i rapporti che ella ha colla condizione dell'attuale stato di società". ${ }^{173}$

La libertà, invero, poteva essere angustiata in molti modi. La angustiavano, per esempio, le requisizioni, le perquisizioni. Era mia intenzione abbandonare il Piano per la caccia, ma il tema perquisizione mi vi riconduce. Un suo articolo (n. 21), infatti, poteva dar luogo, secondo Beccaria, a denunzie occulte, a perquisizioni domestiche e per conseguenza ad odiose inquisizioni, vessazioni indebite e a vendette private per un oggetto, la caccia, appunto, "che vuol essere trattato con dolcezza e di cui è espediente che sia punito il solo contravventore colto in fragranti". ${ }^{174}$ La perquisizione domestica "deve riservarsi per i delitti che sommamente interessano la società e non per delitti di questa natura [quelli, per l'appunto, inerenti alla caccia]" ${ }^{175}$ In ogni caso, le perquisizioni sono, generalmente, "inefficaci, se non sono sostenute con qualche rigore, e sempre contrarie allo spirito d'industria che esige libertà e facilità $[\ldots]$ "; ;76 sono "procedure odiose e gravose al commercio". ${ }^{177} \mathrm{La}$ perquisizione domestica poteva essere ammessa "quando trattasi di un regale d'insigne entità, la di cui conservazione interessi sommamente i diritti del Principato, e per conseguenza anche la società

173 Ibid., p. 38. Siffatti rapporti consistevano nella conservazione della selvaggina, a vantaggio dell'erario, certo, "ma ancora per avere una stabile provista di quest'articolo di consumazione e di commercio, non necessario alla comune sussistenza ma non indifferente ai commodi di una colta società"; nella salvaguardia delle campagne coltivate e, in genere, della proprietà dai danni e dalle turbative arrecati dai cacciatori; nel necessario controllo della detenzione e dell'uso delle armi necessarie alla caccia.

174 Ibid., p. 45 s. L'articolo, secondo Beccaria troppo rigoroso per l'indicato motivo, proibiva "la semplice ritenzione di cani da caccia, uccelli, borse, reti, lacci, archibugi ed altri arredi atti alla caccia [...]". Similmente, l'art. 24, per il quale un capo di selvaggina vivo o morto ritrovato "tanto in casa che fuori" costituiva "senz'altro un corpo di delitto, e il portatore si ritiene per delinquente ipso jure et facto". Per Beccaria, il riferimento al ritrovamento fatto "in casa" doveva essere omesso, in quanto dava "ansa alle sempre odiose inquisizioni domestiche [...]" (ibid., p. 46).

175 Ibid., p. 53.

176 BeCCARIA, Atti di Governo, XI [nt. 9], doc. 3302, 26 ottobre 1789, p. 707.

177 BeCCARIA, Atti di Governo, IX [nt. 19], doc. 1770, 14 maggio 1787, p. 373. 
contribuente [...]". ${ }^{178}$ Sotto questo profilo ammissibili per Beccaria risultavano le perquisizioni effettuate dalle guardie di Polizia che, non mai in numero minore di cinque -anche i campari, si ricorderà, non dovevano operare da soli, ma dovevano essere accompagnati da un altro camparo ovvero da un testimone- compivano tali atti, "principalmente alle cantine dei mercanti di vino trasgressori a' veglianti regolamenti”. Proprio al fine di evitare il malcontento dei perquisiti era previsto che nello svolgimento delle perquisizioni intervenisse sempre uno dei commissari ispettori, il che avveniva in genere nelle perquisizioni che "d'ordinario si fanno o per scoprire contravventori agli ordini o qualche altro corpo di delitto; le quali poi non vengono mai intraprese se non precedono fondate suspicioni per lo meno, dal che si desume insusistente l'esposta doglianza de' cittadini che si vorrebbero far ritenere esposti, nel presentaneo sistema, a capricciose perquisizioni e ad indebite vessazioni con scredito dell'Ufficio medesimo". ${ }^{179}$

A Milano era stato istituito l'Ufficio di Polizia, i cui poteri di strumento locale dell'autorità sovrana erano piuttosto ampi: gli era attribuita una serie di competenze discrezionali nelle quali si ravvisava un illiberale strapotere del Governo in campo giudiziario e il pericolo di indiscriminate vessazioni a danno dei privati. Già si è detto come l'attribuzione della competenza giurisdizionale per i delitti politici alla Police giuseppina avesse sollevato attriti e contrasti con la magistratura ordinaria: a Milano, il rischio di siffatti attriti e contrasti era stato eliminato, apparentemente, ma in realtà acuito attraverso la riunione nella stessa persona, Girolamo Carli, delle diverse funzioni, preposto, com'era stato, sia all'Ufficio di Polizia che al Tribunale criminale di I istanza [Garlati, 2006, p. 504 nota 154].

L'unione al vertice delle due magistrature sollevò comunque dissensi in un'opinione pubblica già scossa dalla partizione giuseppina dei delitti in criminali e politici: tale unione "divenne un simbolo degli arbitrii del sistema giuseppino nella mentalità degli esasperati cittadini milanesi" (Cuccia, 1971, p. 107). Si accentuò l'impressione negativa delle riforme di Sua Maestà, ci si considerava esposti a vessazioni a con-

178 BeCCARIA, Atti di Governo, XI [nt. 9], doc. 2851, 19 gennaio 1789, p. 53.

179 BeCCARIA, Atti di Governo, XII [nt. 62], doc. 3806, Evasione del Dipartimento VI [recte II] all' articolo Ufficio di Polizia. Risposta all'appuntamento n. 16 ed annesso allegato 23 agosto 1790, pp. 482-488, in particolare, p. 486. 
danne arbitrarie, pronunciate sulla base di processi, della cui regolarità procedurale si diffidava assai, dal potere esecutivo che si riassumeva praticamente in una sola persona, il plenipotenziario Wilczeck. E Pietro Verri scriveva: "L'idea era eccellente per prevenire i delitti, conservare il buon ordine nella città, e porvi alla custodia non le infami persone degli sbirri" -secondo Beccaria, come si è detto, costoro manco potevano aspirare a divenir campari- "ma degli onorati soldati resi inabili. L'esecuzione fu veramente bestiale", e riporta esempi della brutalità della polizia che "per conservar l'ordine sociale" non esitava ad adoperare il fucile. E l'adoperò anche nei confronti di un carbonaio che rimase ucciso, nei pressi di Santo Stefano. ${ }^{180}$

Beccaria la pensava diversamente [Capra, 1984, p., 536]. Essenzialmente egli coglie l'aspetto positivo dell'opera di prevenzione svolta dalla Polizia tesa "al bene della civile società". Il fine, al quale in passato tendevano le fonti normative (le "Costituzioni del Dominio di Milano, le Statutarie Leggi Municipali e gli Ordini Sovrani”) oggi è affidato alla rinnovata istituzione: al "bene pubblico" oggi provvede più da vicino il nuovo regolamento di Polizia che disponeva di agili ed efficaci strumenti d'intervento, quali il ruolo degli abitanti, il registro dei forestieri, i posti di guardia alle porte della città, l'assegnazione di un commissario nei sei quartieri della città, le ronde notturne che perlustravano "le strade, i pubblici alberghi ed i luoghi sospetti, onde attrappare le persone equivoche ed impedire gli inconvenienti [...]" ${ }^{181}$ Con tali strumenti più volte si ottenne con ottimo successo di "precluder l'adito al male",

180 P. VerRI, Memoria cronologica de' cambiamenti pubblici dello Stato di Milano dal 1750 al 1791, in ID., Scritti politici della maturità [nt. 152], V Scritti sulle riforme del periodo leopoldino (1790-1791), pp. 559-592, in particolare, p. 586, ove Verri ricorda anche che "questa Police [venne] tolta dopo cinque anni": da Leopoldo II il 20 gennaio 1791 (ibid., nota 72). L'episodio è ricordato anche nei Pensieri del Conte Pietro Verri sullo stato politico del Milanese [nt. 152], p. $401 \mathrm{~s}$.

181 BecCaria, Atti di Governo, XII [nt. 62], doc. 3806, [nt. 179], p. 484. Beccaria rappresenta i rapporti tra le magistrature in modo del tutto positivo, il che sembra essere contraddetto da altre testimonianze. La Commissione politico giudiziaria non aveva "fino ad ora [...] potuto, ad onta delle vaghe accuse contro l'Ufficio di Polizia, citare dei casi specifici, meno provarli, ne' quali abbia l'Ufficio stesso abusato della propria autorità o oltrepassati i confini della propria giurisdizione [...]". Ed ancora: "[...] fra le podestà giudiziarie e le politiche è sempre passata quella buon'armonia necessaria per il bene pubblico e per il buon servigio del Sovrano; hanno sempre mai 
il che costituiva il fine istituzionale della Polizia, ${ }^{182}$ "sotto il qual nome scriveva Beccaria vent'anni prima nel Piano delle Lezioni di Pubblica Economia, ${ }^{183}$ - si comprendono tutte le regole che contribuiscono al buon ordine ed alla facilità di tutti gli affari economici di uno stato: nettezza, sicurezza e buon mercato sono i precipui oggetti di ogni polizia civile: [...] sicurezza nel prevenire i delitti, nell'estirpare l'ozio, nel frenare tutto ciò che disturba la tranquillità pubblica, onde s'arenano i commerci e languisce l'industria che dalla buona fede e dall'impedire le frodi si anima e si mantiene [...]" [Chiappetti, 1973, p. 64 ss.].

Buona fede che deve essere tutelata, prevenzione e repressione delle frodi sono argomenti per i quali Beccaria rivela profonda sensibilità ed ai quali dedicò pagine importanti del Dei delitti e delle pene, degli Elementi di economia pubblica, nonché parte non piccola delle fatiche durate nelle vesti di alto funzionario. Una sensibilità che non deve essere oggetto di meraviglia, quando si pensi al ruolo che la pubblica fede e il credito pubblico svolgono per il fiorire dei traffici e dei commerci e quindi per il buon andamento dell'economia del paese. Il credito pubblico "altro non è che una confidenza e una fiducia che provano i sudditi riguardo agli altri sudditi, i membri di una nazione con quelli di un'altra, di poter sicuramente e facilmente cambiare e contrattare i valori che posseggono con altri che possono desiderare". Il credito pubblico e la facilità, la prosperità di commercio si alimentano l'un l'altro. Se è vero, infatti, che "dalla facilità e promiscuità dei commerci di varie nazioni $[\ldots]$ nasce quel fenomeno politico e morale che chiamasi credito pubblico", altrettanto vero è che "questa medesima confidenza [...] diviene a vicenda cagione di maggior prosperità e facilità del commercio medesimo". ${ }^{184}$ E poiché "gli uomini hanno sovente abbondanza di alcune cose di cui altri sono bisognosi, e scarsezza di alcune altre di cui altri abbondano [...] si permutano dunque reciprocamente,

quelle osservati religiosamente i confini della propria giurisdizione, ed il politico si è sempre fatto il dovuto carico di rimettere alle medesime la cognizione degli affari della loro pertinenza e così viceversa, né sono accadute le esposte confusioni nelle incombenze delle stesse facoltà [...]" (p. 486 s.).

182 Ibid., p. 485.

183 C. BECCARIA, Piano delle lezioni di pubblica economia che si danno nello spazio di due anni dal professore di questa scienza [1771(?)], in ID., Opere, a cura di S. RomaGNOLI, volume primo, [Firenze] 1971, pp. 350-359, in particolare, p. 355 s.

184 BECCARIA, Elementi [nt. 4], p. IV, cap. IX, $₫ 53$, p. 158 s. 
siano le produzioni del suolo, siano le opere della loro industria”, a pieno titolo il commercio, in cui si concreta siffatta permutazione, è "oggetto d'economia pubblica", ${ }^{185}$ definita, lo si è ricordato, come "l'arte di conservare ed accrescere le ricchezze in una nazione, e di farne il miglior uso". ${ }^{186}$

Già si è detto che Beccaria riponeva grande fiducia nella libertà. Libertà che, insieme con la "facilità" era di fondamentale, vitale importanza per lo "spirito d'industria": ${ }^{187}$ così il Beccaria in azione, cui faceva coerente riscontro il Beccaria a tavolino, per il quale, e cito una sola sola frase tra le tante proponibili, "il miglior incoraggimento d'una coltura non può essere che il libero spaccio del prodotto". ${ }^{188}$ Tra i mezzi utili al fiorire dei commerci (economia della mano d'opera, buon mercato nel trasporto, piccoli interessi del denaro), Beccaria indicava anche quello consistente nella "concorrenza del prezzo delle cose", ${ }^{189}$ il che è dire "la massima concorrenza sia dei compratori come de' venditori". ${ }^{190}$ Ebbene, questo mezzo "si ottiene col maggior grado di libertà a tutti di fare quel commercio che più piace, non limitata che da quella disciplina che piuttosto aumenta a ciascuno il potere di ben fare e toglie quello di far male altrui ed alla società". ${ }^{191}$ Così posta la massima dell'assoluta libertà di commercio in materia di grani, le modificazioni saranno opportune e necessarie solo quando tale libertà assoluta risulti dannosa. Ma, in ogni caso, le modificazioni "debbono scostarsi il meno che sia possibile dalla libertà medesima" ${ }^{192}$ E questo, perché? Perché, come spesso accade nei regolamenti annonari, "per li quali talvolta, cercando l'ottimo, si perde di mira il buono, e si corre rischio di perdersi in un labirinto di difficoltà [...]". ${ }^{193}$

185 BECCARIA, Elementi [nt. 4], p. I Principj e viste generali, $\$$ 2, p. 20 s.

186 Ibid., $\mathbb{1} 1$, p. 19.

187 v. retro p. 298 e note $176,184$.

188 BeCCARIA, Elementi [nt. 4], p. II, cap. V, $\$ 40$, p. 179.

189 BECCARIA, Prolusione [nt. 5], p. 172 s.

190 BeCCARIA, Elementi [nt. 4], p. IV, cap. IV, $\$ 30$, p. 95 s.

191 Ibid.

192 Ibid., p. II, cap. V, $\$ 46$, p. 197.

193 BeCCARIA, Atti di Governo, VI [nt. 25], doc. 187 Relazione della Provincia d'Annona compilata dallo spettabile consigliere marchese don Cesare Beccaria Bonesana, relatore del suddetto Dipartimento e dal medesimo letta sedente il Regio Ducale Magistrato Camerale il giorno 11 agosto 1773, pp. 275-356, sul punto, p. 295. 
Nel 1773 era giunto in effetti il tempo di apportare tali modificazioni attraverso un editto, al quale Beccaria pose mano. Posta la necessità di perseguire sia il fine della prosperità dell'agricoltura sia quello della sussistenza del popolo, due erano le vie percorribili. La prima era quella dell'assoluta ed illimitata libertà di circolazione interna e di estrazione del grano; la seconda quella di una libertà limitata da qualche vincolo e cautela. Beccaria, membro al tempo del Magistrato Camerale, abbandona l'idea nella quale pur fermamente credeva, e si fa pragmatico, disposto ad imboccare ed a percorrere la seconda via. Era necessario assicurare l'interna circolazione e, al contempo, la massima possibile libertà d'estrazione, vale a dire, la massima che fosse compatibile con l'esigenza di assicurare la sussistenza del popolo. A tal fine era utile la fissazione di un prezzo limite del grano (quanto si discusse intorno alla fissazione di questo prezzo!), raggiunto il quale, o raggiuntolo nei pressi, si sarebbe dovuto bloccare l'estrazione del cereale. ${ }^{194}$ Ma non solo questo. Leggendo la relazione ci avvediamo che egli si mostra favorevole al sistema delle licenze per il trasporto del grano da provincia a provincia e similmente all'interno della zona di confine di 3 miglia, ritenendo, questo, il solo strumento utile per prevenire il contrabbando. ${ }^{195}$ Giustifica inoltre l'obbligo d'introduzione del grano nelle città, così come quello delle notificazioni, ch'egli considera come "la base di tutto il regolamento annonario", ${ }^{196}$ notificazioni e introduzioni che, insieme con il controllo sulle compravendite di grano, sulla circolazione all'interno della provincia e i vincoli all'esportazione costituiscono le quattro categorie in cui si riassumono le norme e i regolamenti in materia [Capra, 1998, p. 218 s.; Id., 2000, p. 70 s.].

Nell'allontanamento di Beccaria dalla netta e limpida concezione liberista degli Elementi si è voluto cogliere l'effetto della consapevolezza della grave crisi del tempo e del suo far parte di un Consesso, che, presidente Gian Rinaldo Carli, era in massima parte composto da laudatores dell'antico. Un Beccaria, pertanto, già l'ho detto, pragmatico e quindi assai cauto. Mi paiono chiare al riguardo le parole di Melchiorre Gioia: "Risulta dai fatti antecedenti che non si dovrebbero togliere tutte le mete con un colpo. [...] il popolo che ha afferrato la meta co' denti,

194 Ibid., p. 309.

195 Ibid., pp. 299-301.

196 Ibid., pp. 314-316. 
e la ritiene con maggior ostinazione che il cane un osso spolpato; il popolo, dico, realizzarebbe il caso del pupillo che unitosi cogli usurai fa la guerra al suo tutore. L'istruzione deve quindi precedere la legge, la legge dovrebbe essere stesa con quella prudenza che brilla nel decreto 31 dicembre 1771. Questo decreto sciogliendo da ogni pagamento l'interna circolazione del grano, fa sperare maggior libertà nel futuro: permette gli ammassi, ma per rispetto all'opinione, li avvincola con varie cautele; non concede la libera estrazione, ma ne fa travedere la probabilità; spezza tutti que' vincoli, che comunemente erano riconosciuti per dannosi, e promette di spezzarne altri, per trarvi sopra gli sguardi del pubblico". ${ }^{197}$

Ma Cesare Beccaria non rimane fermo nella sua posizione ora descritta: evolve in connessione con l'evolvere del contesto in cui vive ed opera racchiuso [Carera, 1990, pp. 434-446]. Nel 1781 la crisi economica è un ricordo, Gian Rinaldo Carli non è più presidente del Magistrato, sostituito da Pietro Verri [Capra, 2002, pp. 455-464]. E del 28 aprile 1781 - "certamente uno degli atti pubblici più alti predisposti da Beccaria, per impianto e per trattazione" [Carera, 1990, p. 441 s.] è un'importante consulta elaborata in rappresentanza del Magistrato Camerale ove è descritto il funzionamento del sistema annonario in vigore ed è affrontata la questione relativa alla determinazione del prezzo normale del grano. Leggo: "Se è libera la circolazione interna ne' modi sovraindicati, restano ancora alcune interiori cautele e vincoli che

197 GIOIA, Sul commercio de' commestibili [nt. 1], p. 392 s. In BECCARIA, Atti di Governo, XVI-II [nt. 81], Addenda 8.3 si legge il Piano d'Annona (bozza di consulta, 23 dicembre 1771), pp. 978-983, dal quale risulta che "uno dei più premurosi ed importanti oggetti ai quali abbiamo creduto di preciso dovere il rivolgere immediatamente la nostra attenzione fu quello di disporre li mezzi più convenevoli e più innocui per l'esecuzione del nuovo Piano d'Annona, espressamente dalla materna augusta provvidenza di Sua Maestà ordinato affine di combinare la libertà de' grani a vantaggio della nazione colle cautele necessarie al comune alimento" (p. 978 s.). Sul "cambiamento di sistema seguito alla fine del 1771" in materia di annona si veda la Relazione 11 agosto 1773, citata in nota 193 , p. 275 ss. Le cautele previste dall'editto 31 dicembre 1771, dieci anni più avanti, erano considerate "sovverchie": "anzi oso dire erano contrarie al grande oggetto della legislazione de' grani che deve spingere le speculazioni degli uomini al commercio continuo e sempre vivo di questa derrata, e non restrigersi alle minute cure regolatrici dell'economia di una privata famiglia" (BECCARIA, Atti di Governo, VII [nt. 139], doc. 648 Nuovo piano annonario, 28 aprile 1781, pp. 456-483, in particolare, p. 459 s.). 
potranno sembrare a molti più atte a rallentare la celerità della circolazione e a raffreddare il calore con cui deve procedere un commercio, che è l'oggetto dei bisogni e delle ricerche di ogni ordine di persone in ogni tempo e in ogni luogo, piuttosto che a procurare l'abbondanza e per conseguenza il buon mercato della derrata". ${ }^{198}$

Beccaria, a differenza di quanto sostenuto nel 1773, è ora assolutamente contrario alle leggi che prevedono e disciplinano la notificazione e l'introduzione dei grani nelle città: "leggi che non osservate offendono l'autorità del legislatore ed osservate offendono una gran parte della popolazione, cioè li proprietari e li coloni, esponendoli al capriccio o per lo meno alla negligenza ed agli sbagli inevitabili degli esecutori [...]". ${ }^{199}$ La descrizione del "nostro Piano attuale d'Annona" è stata tracciata nelle sue linee di fondo in quanto utile nel rendere più agevole "l'esposizione del mio sentimento tendente alla più placida esecuzione del nuovo Piano comandato da Sua Maestà. Lo stabilimento di un prezzo regolatore della proibizione o della libertà dell'estrazione de' grani e la soppressione dei vincoli inutili per l'interna circolazione sono i due cardini intorno ai quali secondo la sovrana mente debbono agirarsi le nostre riflessioni. Tutti quelli che hanno profondamente meditato e scritto su questo importante argomento hanno ridotto lo stato della questione a ricercare se, in vista del doppio fine della prosperità dell'agricoltura e della sicurezza della sussistenza del popolo, sia preferibile una assoluta ed illimitata libertà, oppure qualche vincolo o cautela che limiti la contrattazione in una sfera più ristretta. Hanno deciso in favore della totale libertà colla soppressione dei vincoli di qualunque

198 Ibid., p. 458.

199 Ibidem. Particolarmente severo sarà Melchiorre Gioia nei confronti de "gli agenti, o ispettori, o commissarii dell'annona, o capitani del divieto, o comandanti di piazze, o generali di provincie o chi che altro alla circolazione del grano soprintende, o vuol soprintendere. Queste onestissime persone, trovando cosa giusta di mettere a profitto il loro tempo e potere [...] questi vigilanti e probi magistrati, che spargendo minaccie d'accuse e sequestri, traggono nelle loro reti l'ignoranza e la debolezza; che, profittando del timore da essi diffuso, parlano alto, e van sulle furie, e gridano al contrabbandiere, al monopolista, al ladro; tanta è in essi la certezza che sono rari i loro simili! Che finiscono col farvi imprigionare, per dimostrarvi coll'ultima evidenza che, avete torto di chiedere il vostro grano, gli agenti dico dell'annona sanno far conoscere mille occasioni, onde procurarsi sempre nuovi guadagni" (GIOIA, Sul commercio de' commestibili [nt. 1], p. 363 s.). 
sorta, dimostrando che questi rendono meno avantaggiosa la coltura de' grani, ne diminuiscono l'annua riproduzione, che perciò si oppongono allo scopo per cui sono stati istituiti, cioè di procurare la mediocrità de' prezzi in favore de' consumatori, e costringendo il legislatore a moltiplicare sempre più li ceppi a questo commercio per opporsi a tale tendenza; hanno provato che la sussistenza o il rinovellamento periodico di questi vincoli, tenendo avviliti li prezzi nei tempi del raccolto, accumulano facilmente $i$ grani in mano di pochi; questi se non altro approfittano dei brevi momenti di libertà, che pure conviene concedere talvolta per ismaltire il superfluo, ne fanno uscire grandiose quantità in una volta, per cui nasce poi facilmente il timore della carestia. Queste ed altre molte riflessioni, che sarebbe qui troppo lungo l'esporre, messe nella più chiara luce dai più accreditati scrittori, fra i quali posso, appoggiato al pubblico giudizio dell'Europa, annoverare chi degnamente ci pressiede, da alcuni Parlamenti di Francia, dalla sperienza di varie nazioni, e principalmente dalla Toscana, hanno fatto concludere che la totale libertà non producané quella mancanza di grano né quell'eccesso di prezzi che hanno riconosciuto per causa loro i vincoli e le cautele" ${ }^{200}$ Beccaria riconosce che la libertà di commercio introdotta "d'un balzo" avrebbe potuto avere effetti negativi: più opportuno, pertanto, era concederla per gradi. A tal fine tra le "circoscrizioni" più utili, in quanto tra "le più semplici e le meno angustianti il commercio de' grani" e per "dimostrare al pubblico che resta in ogni straordinario evento assicurata la sussistenza, senza vincolare il commercio che può solo operare colla libertà", è stata prescelta quella consistente nella "fissazione di un prezzo normale". Strumento utile, certo, ma che sarebbe potuto andare incontro a una difficoltà: "[...] aumentandosi i prezzi de' grani ed accostandosi sempre più al limite di quel prezzo oltre il quale fosse proibita inesorabilmente l'estrazione, tutti i possessori di grano tenderanno a rinserrarlo e tenerlo fuori di contrattazione per riserbarlo fino al maggior prezzo possibile, e poi lo faranno uscire tutto in una volta, quando questo sia giunto vicino al detto prezzo normale, per approfittare della spirante libertà e sottrarlo dall'imminente proibizione". Il modo per controbattere questo pericoloso inconveniente stava nella determinazione di un prezzo normale piuttosto alto "di modo che

200 BeCCARIA, Atti di Governo, VII [nt. 139], doc. 648 Nuovo piano annonario 28 aprile 1781, p. $467 \mathrm{~s}$. 
fosse rimoto il pericolo della sovraindicata dannosa accelerazione dell'uscita de' grani" ${ }^{201}$

Beccaria sviluppava poi i contenuti del nuovo Piano di regolamento annonario in otto punti, l'ultimo dei quali era redatto in questi termini: "Ritenute le sufficienti cautele oggi veglianti per il più volte detto circondario [di tre miglia de' confini], io non crederei opportuno di accrescere le penali contro li fraudolenti estrattori nella pubblicazione di un nuovo sistema tendente a dare maggior libertà e vigore a questo commercio, molto meno a facilitare il metodo dei processi inquisizionali, i quali scoraggiscono l'industria e rare volte conducono a qualche risultato di provata reità" ${ }^{202}$ In una nota a margine del verbale della riunione si legge: "Fu detto: Commendata la prontezza, cautela ed esattezza del Relatore, si rassegni lo scritto mediante consulta a Sua Altezza Reale con le osservazioni fatte in Tribunale" ${ }^{203}$

Il 16 gennaio 1793 Beccaria, nello stendere una consulta in risposta ad una serie di quesiti posti dalla "Reale Corte di Toscana" in materia di "Annona e Vettovaglie veglianti nella Lombardia Austriaca", precisava che la materia risultava regolata dal testo 4 aprile 1786 (Regolamento per il commercio de' grani della Lombardia Austriaca). ${ }^{204}$

201 Ibid., pp. 468-476. Il prezzo limite - esclusa la possibilità di prezzi 'normali' differenti da provincia a provincia, come misura che avrebbe contraddetto il principio della libera circolazione interna - era da Beccaria fissato in lire 43 e soldi 10, al quale si perveniva sulla base del "medio aritmetico risultante dai rispettivi prezzi maggiori dei detti quattro mercati", vale a dire dei mercati di Milano (Broletto), Varese, Como, Laveno. Qualora in due di questi mercati fosse stato superato il prezzo limite, le esportazioni avrebbero dovuto essere vietate. L'editto 4 aprile 1786 avrebbe fissato in lire 36 il prezzo limite per il frumento, prezzo che "non era affatto alto" (Grab, 1982, p. 463; 1986, p. 174 s.).

202 BeCCARIA, Atti di Governo, VII [nt. 139], doc. 648 Nuovo piano annonario 28 aprile 1781 , p. 482.

203 Ibid., p. 483 nota 1.

204 Lo si legge in Atti di Governo, VIII [nt.19], doc. 1277, 24 marzo 1786, alle pp. 605-614. Nel preambolo ricorrono le seguenti parole: "Dopo che cogli editti 31 dicembre 1771 e 10 febbraio 1776 si sono accordate varie facilitazioni per far prosperare l'importante ramo dell'Annona nello Stato di Milano, e dopo di avere tolto il vincolo delle annuali notificazioni e introduzioni de' grani nelle rispettive città [...] Sua Maestà l'Imperatore e Re nostro clementissimo sovrano si è degnato ordinare che non solo tolgansi dall'interno di tutta la Lombardia Austriaca li vincoli e pagamenti finora cadenti sopra i grani di qualunque sorte e sul riso, ma ben ancora che sino ad un dato limite di prezzo per 
Fondamentalmente vigeva "la libertà dei grani per uscita entrata e transito". Il Governo interveniva soltanto quando il prezzo del grano fosse asceso a lire 36. Questo prezzo - il "prezzo normale del grano" - non determinava di per sé la sospensione dell'uscita del grano stesso, ma dava al Governo la facoltà di intervenire ove lo "esigesse la sicurezza della pubblica sussistenza". Siffatto regolamento "ha avuto il più prospero successo", precisava Beccaria, facendo riferimento alla "tabella [...] delli adequati dei prezzi fatti in Milano tra il 1781 e il 1791". Nel primo quinquennio il prezzo medio fu di lire 32, nel secondo di lire 30.13.2, "cioè qualche cosa di meno dopo gli anni di libertà". ${ }^{205}$

Prima di allontanarmi dal Beccaria liberista, cauto liberista in materia, mi piace ricordare come Melchiorre Gioia, nello scrivere Sul commercio dei commestibili avesse sotto gli occhi la relazione beccariana sulla quale mi sono prima soffermato Con riferimento al periodo 1779-2 maggio 1781, Gioia ricordava come fosse stata sostanzialmente introdotta la libera circolazione dei grani, il che comportò non innalzamento dei prezzi, ma "una graduata diminuzione, il che prova, soggiunse Beccaria, relatore di questo fatto" - sto qui leggendo Gioia -, "che la facilità non interrotta del commercio dei grani, se non sempre diminuisce, non fa almeno alzare i prezzi” ${ }^{206}$ Sempre nello stesso scritto, esponendo le proprie concezioni assolutamente liberiste, Gioia si faceva forte del pensiero di Pietro Verri e di Beccaria. "Spero peraltro qualche speditezza, giacché il marchese Beccaria e il conte Verri, che

ciascuna qualità di detti grano e riso [...] ne sia libera l'estrazione dallo Stato sotto una mite porzione di surrogato da pagarsi al solo confine, affinché non venga esposto il Regio Erario a un grave sacrificio e da un altro lato sciolto possibilmente il commercio de' grani ne' suoi rapporti tanto interni che coll'estero, possa ottenersene quella prosperità che rendesi tanto necessaria alla floridezza dell'agricoltura ed al bene pubblico". L'art. XXV (p. 611) fissa il prezzo limite nella misura di cui alla nota 201.

205 BeCCARIA, Atti di Governo, XV [nt. 47], doc. 5085, 16 gennaio 1793, pp. 2633, sul punto, p. 28 s. "Come consigliere, Beccaria avrà occasione di intervenire di nuovo su di una specifica questione annonaria, la panificazione, ma l'irripetibile competenza maturata negli anni della liberalizzazione potrà tornare utile solo nel settembre 1792, quando all'anziano ministro si ricorre perché nessun altro è in grado di rispondere soddisfacentemente ai quesiti formulati dalla Corte di Toscana interessata alla normativa milanese" [Carera, 1990, p. 444].

206 GiOIA, Sul commercio de' commestibili [nt. 1], p. 424 s. Gioia aveva dunque sotto gli occhi la relazione beccariana del 28 aprile 1781, sulla quale v. testo corrispondente alle note 197-203. 
sono del mio parere, spalleggieranno la mia domanda". E cita, di Verri, il Discorso sulle leggi vincolanti e, di Beccaria, "vari discorsi inediti recitati avanti il Magistrato Camerale, esistenti nell'Archivio Nazionale". ${ }^{207}$ Non pochi sono i passi testualmente riportati, con la dovuta ed esatta citazione. Non era nel vero Vincenzo Cuoco laddove aspramente criticava l'opera di Gioia definendola "triviale" e condannandola come un sostanziale e continuo plagio del pensiero dei due illuministi. ${ }^{208}$

Ho detto di Gioia liberista convinto, senza comunque dimenticare che nella sua vita culturale ad un periodo "dominato da una concezione quasi mistica della libertà" succedette quello "caratterizzato da una viva simpatia per un intervento attivo dell'autorità govenativa negli affari economici" [Barucci, 1965, p. 117 s.; Romani, 1990, pp. 310-316, Porta e Scazzieri, 1996, pp. 36-40]. Richiamo l'attenzione su di una sola pagina. "Parlando della libera circolazione del grano conviene osservare ch'ella, dirò così, imbriglia i prezzi, e non permette

207 Ibid., pp. 371, 374 e nota 5, rispettivamente. Le verriane Sulle leggi vincolanti principalmente nel commercio de' grani. Riflessioni adattate allo Stato di Milano coll'occasione che trattasi di riformare il sistema d'annona, redatte nel 1769, vennero riposte nel cassetto "[...] e vi rimasero fino al 1796, quando il Verri deciderà di darle alle stampe in un contesto politico totalmente mutato" [Capra, 2002, p. 303]. Si leggono ora in P. VERRI, vol. II tomo II dell'Edizione Nazionale delle Opere [nt. 133], I Scritti sull'annona e sui prezzi. Testi a cura di R. Pestarino. Commento di G. Tonelli, pp. 247-371. Verri esprime netta avversione contro il sistema attuale, che prevede la notificazione e la introduzione dei grani, la proibizione del loro ammasso, limitazioni al numero e alla libertà dei mercanti, un sistema in cui il "Il trasportare grani fuori dello stato è un delitto per cui s'intima la pena di morte: condurre il grano da un distretto all'altro nello Stato medesimo è un delitto, per cui vien minacciata la perdita de' grani e degli attiragli [...]" (parte seconda, p. 293 s.). Premesso che qualsivoglia temperamento alla libertà è un male, Verri scrive: "La libertà del commercio de' grani, dicono alcuni, è una legge opportuna per quegli Stati che non hanno perfezionata l'agricoltura; la libertà della contrattazione de' prodotti è un mezzo efficacissimo di promuoverla; ma promossa ch'ella sia alla sua perfezione, come lo è da noi, la libertà non ha più nessun fine, ed altro non può cagionare se non la carezza del prezzo a scapito della Nazione. Io credo che siasi dimostrato falso quel principio che la libertà tenda ad incarire il prezzo; anzi credo che sia provato e dalla ragione e dagli esempi che si sono riferiti, che la libertà produce i prezzi minori di quello che non fanno le costrizioni e i vincoli, onde la libertà è la tutrice della pubblica abbondanza, non meno che dell'industria nell'agricoltura [...]" (p. 324).

208 V. CuOCo, Lettera ad un amico sull'opera di Gioja del Commercio de' commestibili e caro prezzo del vitto (1802), cito da Nutini, 1989, p. 52. 
loro di fare tanti salti bizzarri dall'alto in basso, dal basso in alto, da un mercato all'altro, da paese in paese, come avviene quando la circolazione è avvincolata. Siccome l'acqua che va per un canale, il cui fondo è sempre piano, sempre parallelo all'orizzonte, cioè sempre alla medesima altezza, marcia regolarmente; siccome all'opposto l'acqua che scorre per un altro canale il cui fondo ora monta, ora discende, qui è intersecato da enormi sassi, là serrato da una chiusa, siccome dico quest'acqua ora s'alza, ora s'abbassa, ora corre precipitosamente, or non si move che a stento; così i prezzi del grano, quando la circolazione è libera, procedono regolarmente; quando è impedita vanno a salti variabili e irregolari. Ora questi salti portano danno principalmente alla classe de' poveri artisti, perché mentre non possono trar profitto dal momentaneo abbassamento de' prezzi, giacché i monopolisti sanno risparmiar loro la pena; all'opposto quando i prezzi montano, gli artisti ricevono bensì come pria la stessa mercede, ma non possono comprare che la metà del pane che compravano dapprima, e dieci soldi non valgono che cinque. Ma quando la circolazione è libera, le mercedi seguono un corso così regolare come i prezzi, e l'entrate e l'uscite si tengono in equilibrio". ${ }^{209}$

Altrettanto significativo è quanto Gioia scrive in materia di concorrenza. "Ma abbiamo detto e provato di sopra, che quanto più crescono $\mathrm{i}$ venditori, tanto più i prezzi s'abbassano, quindi allorché regna la libertà, accade ai prezzi ciò che accade ad una palla posta sopra un piano, ed abbandonata a se stessa; dopo qualche piccola oscillazione ella va da sé a posarsi nel luogo più basso. Ora vi par mo un gran tratto di sapienza il voler ordinare con una legge ciò che avviene naturalmente, necessariamente? Che direste d'un legislatore, che spedisse ordine alle acque che sono sui monti di scendere nelle valli? Mi pare che direste che il di lui cervello si è imbarcato pel concavo della Luna, e munireste il nostro legislatore d'una lettera di raccomandazione pel medico della Senavra. Dunque invece di dire, a cagione d'esempio, il prezzo de' macheroni sarà 9 soldi la libbra, si doveva dire: sia permesso a ciascuno il fabbricare e vendere

209 GIOIA, Sul commercio de' commestibili [nt. 1], p. 362 s. "[...] in materia di commercio dei grani, Gioia non mutò affatto opinione e le molte pagine incluse sulla questione nel Prospetto [...] contengono ancora un'accorata perorazione della più ampia libertà nel commercio dei grani e nella fabbricazione del pane” [Barucci, 1965, p. 118]. 
macheroni a suo piacimento; applicate lo stesso agli altri commestibili; e vedrete che la meta è affatto inutile". ${ }^{210}$

Ma ritorniamo ora a Beccaria liberista. Di certo, qualche amaritudine la provò, per esempio, in materia di produzione e vendita del pane. "[...] credo che miglior legge intorno alla panizzazione non vi sia di questa, faccia pane chi vuole e sia punita la frode [...] Ma nel sistema della libera panizzazione sono assai meno da temersi le frodi che nel sistema della circoscritta". E poi, "libera essendo la panizzazione, la frode vien punita più facilmente da se stessa [...] l'interesse otterrà ciò, che le più severe ordinazioni non ottengono", così scriveva Beccaria negli Elementi. ${ }^{211} \mathrm{Ma}$ la libera panizzazione incontrava difficoltà nella realtà locale: a Como, per esempio, si proponeva di istituire, per sperimentazione, prima uno, poi due "prestini di campione"; ${ }^{212}$ similmente a Bozzolo $;^{213}$ a S. Martino era stato reintrodotto il calmiere. ${ }^{214}$ A Mantova, nel 1787, la Congregazione municipale creava non poche difficoltà alla libera panificazione che "ha potuto felicemente sostenersi qui a Milano e nelle altre città dello Stato". Perché non "incoragire uno o più intraprenditori che, senza togliere la libertà della panizzazione, aprano qualche forno sotto l'immediata vigilanza della Congregazione o dell'Intendenza politica, come si è praticato qui in Milano, con felice successo, col mezzo di comunità religiose che hanno aperto pubblici prestini?" 215 L'Intendente politico - il conte Giovan Battista Gherardo d'Arco [Mozzarelli, 1990, pp. 79-81, 84] - era d'accordo e trovava esagerate le opposizioni della Congregazione che si sostanziavano in questo: l'erezione di prestini da campione comportava una troppo elevata

210 GIOIA, Sul commercio de' commestibili [nt. 1], p. 386.

211 BECCARIA, Elementi [nt. 4], p. II, cap. V, \$ 62, pp. 219-222.

212 BeCCARIA, Atti di Governo, IX [nt. 19], doc. 1627, 26 febbraio 1787, p. 140; doc. 1642, 5 marzo 1787, p. 157 (riscontro al n. 1230); doc. 1721, 23 aprile 1787, p. 309; doc. 1736,30 aprile 1787, p. 327 s.: "Si potrebbe in conseguenza sperimentare per un anno, e vedere quale vantaggio si può sperare dalla libera panizzazione tenuta in freno dal prestino di campione [...]".

213 Ibid., doc. 1563, 22 gennaio 1787, p. 56 s.; doc. 1663, 15 marzo 1787, p. 192; doc. 1718, 23 aprile 1787, p. 304 s.: "Quantunque Bozzolo sia di una città capo provincia, ad essa non sono applicabili i principii che hanno potuto permettere la libera panizzazione nelle altre più considerabili città della Lombardia [...]".

214 Ibid., doc. 1625, 19 febbraio 1787, p. 137.

215 Ibid., doc. 1614, 19 febbraio 1787, p. 124 s.; doc. 1842, 25 giugno 1787, p. 472 s. 
spesa pubblica e poi questi prestini, praticando prezzi più convenienti avrebbero danneggiato i fornai già operanti sul mercato. La seconda opposizione era dissolta dalla considerazione: se qualcuno non regge la concorrenza può cambiare professione. Quanto alla prima obiezione, si ribatteva che non era il caso di parlare di aggravii per l'Erario, in quanto per l'istituzione di due nuovi prestini si erano dichiarati pronti i padri domenicani, che però chiedevano un prestito, e i monaci di $\mathrm{S}$. Benedetto, pronti a vendere l'argenteria superflua. Beccaria sembra soddisfatto: che l'Intendente politico si accordi con i due Ordini in modo da erigere questi prestini liberamente, così si mette "freno all'ingordigia de' fornai mantovani”. ${ }^{216}$ Tosto arriva però la doccia fredda: "La Commissione ecclesiastica ha le sue eccezioni e motivi di sperienza per non concorrervi alla permissione" ${ }^{217}$

E a Milano? A Milano la libera "panizzazione" era stata introdotta "felicemente", come ricorda nel 1787 Beccaria. ${ }^{218}$ Lo era stata sei anni prima con editto 17 dicembre $1781 ; ;^{219}$ l'anno precedente, 4 marzo 1780, a Cremona; pochi mesi innanzi, 27 luglio 1781, a Lodi [Grab, 1986, p. 157]. Non erano, pertanto, trascorsi molti mesi dal tempo in cui Beccaria aveva espresso l'auspicio che fosse "eseguibile anche in questa fortunata provincia quanto attualmente si pratica in Toscana" e che si introducesse "una assoluta e illimitata libertà, sia col sopprimere le privative de' prestini coll'abolire le mete alla panizzazione, sia col togliere ogni cautela ed ogni vincolo non solo per li confini ma anche per l'estrazione dallo Stato di tutti i grani [...]". ${ }^{220}$ L'editto prevedeva che ognuno potesse produrre qualsivoglia tipo di pane, prevedeva l'abolizione delle giurisdizioni, della meta e che il pane potesse essere introdotto in città dall'esterno. La riforma dell' 81 aboliva di fatto la Corporazione dei fornai, abolizione che in forma esplicita avverrà nel marzo 1787, tempo in cui si provvide alla soppressione "delle tuttora sussistenti Università e Corpi mercimoniali

216 Ibid., doc. 1909, 6 agosto 1787, p. 579; doc. 2010, $1^{\circ}$ ottobre 1787, p. 696 s.

217 Ibid., doc. 2121, 26 novembre 1787, p. 831 (riscontro al n. 1897).

218 V. retro, nota 215.

219 Milano, Archivio Storico Civico, Materie, cart. 707. Cito da Grab, 1986, p. 157 e nota 14 di p. 181.

220 Beccaria, Atti di Governo, VII [nt. 139], doc. 648 Nuovo piano annonario, 28 aprile 1781, p. 483. 
[...]" ${ }^{221}$ Era la logica conseguenza dell'applicazione del principio che aveva ispirato Beccaria nel proclamare "faccia pane chi vuole e sia punita la frode", principio ch'era il nocciolo della "miglior legge intorno alla panizzazione". Ed era quel principio di libertà, che, acquisendo carattere di generalità, aveva ispirato la netta avversione dei Lumi contro il sistema vincolistico delle corporazioni medievali. ${ }^{222}$ Accanto a Beccaria si poneva Pietro Verri, per il quale "L'effetto solo adunque che questi corpi producono si è quello di diminuire il numero de' venditori interni, conseguentemente accrescere il prezzo delle merci, diminuire il numero de' contratti, frenare l'attività dell'industria, e scemare l'annua riproduzione". ${ }^{223}$

Che ogni cosa non marciasse a dovere lo dimostrano alcuni interventi della Corte viennese. Kaunitz, in particolare, invitava a riflettere sulla materia, in quanto l'Intendente generale di Finanza aveva constatato che i prestinai di Milano mischiavano al frumento $1 / 4$ di segale, vendendo di poi il pane come se fosse di solo frumento. Il che ridondava a danno del pubblico, certamente, ma anche della Finanza, perché comportava un mancato introito per la regalia della macina di L. 107.000 annue. ${ }^{224} \mathrm{Nel}$ marzo 1789, la Corte invitava Beccaria a raccogliere pareri sulle conseguenze negative della libertà di panificazione soprattutto per

221 Beccaria, Atti di Governo, IX [nt. 19], doc. 1548 Piano per la soppressione delle Università, 8 gennaio 1787, pp. 26-32, ma v. anche doc. 1689, Attività del Dipartimento III nel 1786 (relazione, 31 marzo 1787), pp. 234-236, nonché doc. 1698 Piano per la soppressione delle Università 2 aprile 1787, p. 281 [Capra, 1984, p. 479 s.]. Una prima 'ondata' di soppressioni, riguardanti 41 corporazioni su 103, venne realizzata tra il 1773 e il 1775 [Grab, 1986, pp. 157-159].

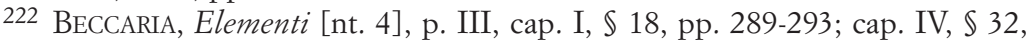
pp. 329-333. V anche retro, testo corrispondente alle note 127, 189-191, 211 e p. 372 ov'è un Beccaria che pone l'accento sulla concorrenza.

223 VERRI, Meditazioni [nt. 133], $\mathbb{S}$ VII De’ corpi de' mercanti e artigiani, pp. 426431, in particolare, p. 428 e, poco oltre: "La libertà adunque nel commercio de' grani non può giammai in nessuno Stato, in nessuna circostanza portar nocumento né alla sussistenza, né all'abbondanza della Nazione. Né possono mai essere di giovamento gli ordini costringenti delle leggi. Se si dubiti della verità di questi principj se ne appelli la decisione alla sperienza, e si ritroverà che gli Stati che non hanno né corpi d'arti e mestieri, né leggi vincolanti all'uscita de' loro prodotti sono più floridi e opulenti degli altri, ne' quali tai organizzazioni coercitive sussistono, e tanto più s'accostano i passi all'ubertà e all'abbondanza, quanto meno sì fatte leggi si tengono in vigore" (ibid., $\mathbb{S}$ IX, Della libertà del commercio de' grani, pp. 433-440, in particolare, p. 440.

224 BeCCARIA, Atti di Governo, X [nt. 37], doc. 2658, 9 settembre 1788, p. 586. 
le classi di piccoli salariati, giornalieri, artigiani. Occorreva, pertanto, intervenire "affine di combinare, se fia possibile, la già da varii anni introdotta libertà di panizzazione coll'altro non meno importante oggetto di provvedere i poveri di un pane sano e a buon mercato". 225 Di lì a tre mesi (giugno 1789), la Corte incalza ancora Beccaria: non era dubbio che la libera panificazione fosse stata introdotta con cognizione di causa e per vantaggio del pubblico, ma altrettanto indubbio era che essa aveva prodotto effetti contrari a ciò che si era previsto ed auspicato: il prezzo del pane venale è aumentato anziché diminuito. Pertanto, sollecita che si intervenga in modo da regolare i provvedimenti sulla base dell'esperienza di più anni. ${ }^{226}$ Beccaria naturalmente risponde: si stava pensando ad un sistema che, "senza togliere direttamente la libertà della panizzazione", ottenesse "il fine salutarissimo della lettera ministeriale 11 dicembre dell'anno scorso [1788], cioè di dare al popolo [...] un pane sano ed a buon prezzo [...]". "Senza togliere direttamente la libertà", sottolineava Beccaria, perché dopo l'editto del 1781 si sono moltiplicati sempre più i prestinai sino a raggiungere il numero di 166 . Di questo dato occorreva tener conto, "quantunque possa esser vero che un tal numero di prestinari non produca l'effetto sperato della concorrenza nel prezzo del pane, benché possa produrlo nella qualità che non è il principale oggetto che vuolsi avere di mira, dove trattasi di sminuita consumazione del popolo [...]". Per i 166 fornai altro mezzo non v'era che "tener alto il prezzo o diminuirne il peso reale, o mascherandolo con una cattiva cottura se non vogliono rovinare tutto". Occorreva tener conto, altresì, che si trattava di 166 fornai che in buona fede hanno impiegato i loro capitali: una subitanea mutazione di sistema e revoca assoluta della legge emanata rovinerebbe le loro famiglie e ridurrebbe la "panizzazione" nelle mani di pochi. Beccaria proponeva i seguenti rimedi. A) trovare cinque o sei facoltose persone che nei principali quartieri si obbligassero a dare pane a meta e sotto le leggi municipali contro qualche ricompensa o facilitazione del Pubblico; B) che ai rivenditori di pane prodotto da altri ("postari") non fosse lecito che vendere pane di meta, mentre la vendita di altro pane avrebbe integrato una fattispecie di contravvenzione; C) senza togliere ai fornai la libertà di fabbricare e vendere a qualunque prezzo e qualità,

225 BeCCARIA, Atti di Governo, XI [nt. 9], doc. 2935, 16 marzo 1789, p. 212.

226 Ibid., doc. 3048, 2 giugno 1789, pp. 347-351, in particolare, p. 347 (n.10780/948: osservazioni della Corte al protocollo del III Dipartimento, relative al n. 614). 
limitare tale facoltà al proprio prestino. ${ }^{227}$ Quanto al punto A, un anno dopo, si rilevava che il suggerito rimedio non era risultato attuabile in quanto non si era trovato un numero sufficiente di persone che avessero voluto addossarsi un tale peso, non solo, ma anche perché "nemmeno con ciò si sarebbero prevenuti i riclami del pubblico per la ragione che se qualche malcontento fosse entrato in un prestino ove non v'è la meta, e che avesse trovato pane cattivo, avrebbe, senza riflettere che vi sono i prestini obbligati a meta, eccitati quei disgustosi clamori[...] che sicuramente verranno sopiti tosto che il pubblico saprà che non vi sono più prestinari che possino ad arbitrio ed impunemente defraudarli". ${ }^{228}$ Si susseguirono i progetti, il progetto Donadeo, il progetto Veronesi, il progetto Cavenago: quest'ultimo lasciava la libertà di fare il pane a chi volesse, purché avesse i requisiti richiesti. Non posso ora entrare nel cuore della questione. Mi pare comunque opportuno rilevare come Beccaria fosse pur sempre fiducioso nella forza libertà, la quale però trovava nel suo processo di attuazione, anche nel settore considerato, forti ostacoli e intralci. Del resto Beccaria stesso rilevava e proponeva: "L'esperienza ha dimostrato che sebbene il pane sia di buon peso e di ottima qualità, vi è sempre qualche indiscreto che eccita nel pubblico qualche malcontento all'effetto che ciascuno sia abilitato a conoscere la legalità del peso del pane, crederei assai opportuno che i prezzi de' grani fossero settimanalmente da esporsi al pubblico sugli angoli della città per mezzo della Congregazione Municipale”. ${ }^{229}$ E' significativo che venga richiamato uno strumento 'antico'.

227 Ibid. Con riferimento a quanto Beccaria sottolinea nella consulta, vale a dire che la libertà di panificazione non comportò già i vantaggi della concorrenza, con il moltiplicarsi sino al numero di 166 dei punti di vendita, quanto piuttosto, stante la stazionarietà della domanda ("la consumazione di circa 120 mila moggia nei prestini a un di presso costante [...]”, p. 348) e dovendo vivere sul guadagno della fabbricazione le 166 famiglie, la conclusione di accordi fra i produttori (v. anche, nello stesso senso, BeCCARIA, Atti di Governo, IX [nt. 19], doc. 1689, Attività del Dipartimento III nel 1786 (relazione, 31 marzo 1787), pp. 260-263), è stato scritto: "L'impossibilità della domanda interna, sia urbana che rurale, di espandersi, spiega la complessa situazione così ben sfruttabile dagli interessi costituiti prevalenti, come spiega gli sfoghi dei consumatori incapaci di veder chiaro, ma capaci di cogliere le posizioni di privilegio [...]" [Romani, 1977, p. 192 nota 176].

228 BeCCARIA, Atti di Governo, XII [nt. 62], doc. 3743, 19 luglio 1790, pp. 388-393. 229 Ibid., p. 393. 
Due anni e mezzo più tardi, Beccaria, facendo il punto sulla situazione, rilevava che la libertà di panizzazione introdotta "nelle città dello Stato negli anni precedenti il 1790, con buon successo bensì, ma non secondato dalla oppinione popolare" trovava applicazione nella sola città di Cremona. Nel 1790 il Governo e la Corte ritennero di dover aderire "alle istanze di qualche pubblico" perché fosse ripristinato il precedente sistema del "pane tariffato". A ciò provvide - "fu poi concesso, piutosto che ordinato, generalmente a tutto lo Stato" sottolinea Beccaria - Leopoldo II con dispaccio 20 gennaio 1791 introducendo un "metodo, che è un partito di mezzo fra la libertà assoluta e i vincoli della panizzazione", un metodo che "cammina con prospero successo in questa vasta capitale $[\ldots] "{ }^{230}$

Il ritratto che queste pagine, per quanto brevi, disorganiche, non poco 'tumultuose', ci consegnano di Cesare Beccaria, pare comunque sufficientemente compiuto. Conosciamo un Beccaria attivo, energico, alacre, "di petto" per usare una sua espressione riferita alla personalità che avrebbe dovuto connotare un perito tessile all'altezza [Pasta, 1997, p. 49]. Insomma un Beccaria che i giudizi su di lui espressi, tra altri, dal Cremani (aveva "mente e cognizioni, ma gli manca l'attività, e l'energia [...]"), ${ }^{231} \mathrm{da}$ Leopoldo II in persona ("onesto, abile, letterato ed esatto, ma poco applicato. Lavora poco, debole assai"), ${ }^{232}$ ben difficilmente lascerebbero presumere [Capra, 1998, pp. 215-217; Id., 2000, p. 67-69].

$\mathrm{E}$ conosciamo un Beccaria ben diverso da quello finemente rappresentato da Giuseppe Rovani: "In altra parte poi [del salotto Ottoboni] Cesare Beccaria, seduto solo, anzi sdraiato su d'un canapè, già annoiato dal peso della sua precoce corpulenza e della gloria che

230 BeCCARIA, Atti di Governo, XV [nt. 47], doc. 5085, 16 gennaio 1793, 26-33, ove (p. 32) si legge: "Questo metodo, che è un partito di mezzo fra la libertà assoluta $e$ i vincoli della panizazione, camina con prospero successo in questa vasta capitale, ma non si è potuto ancora far adottare volontieri dalle altre amministrazioni provinciali, ai quali è affidata immediatamente la direzione delle Vittovaglie".

231 L. CREMANI, Relazione di alcuni oggetti, stabilimenti, e circostanze politiche de’ paesi Lombardo-Austriaci del Professor Cremani, allegato n. 69 alle Osservazioni leopoldine [nt. 42], karton 23, $\$ 30$, f. 391v.

232 Avendo Sua Maestà domandato $i$ Ruoli di tutti gli impiegati di Lombardia, si danno qui annessi, colle osservazioni fatte su i medesimi dalla medesima. Tabella B: Ruolo del R. Magistrato Politico Camerale, allegato n. 72 alle Osservazioni leopoldine [nt. 42], karton 23, f. 414r. 
non aveva cercato, dissimulava, sotto l'aspetto d'una indolenza invincibile, l'attività prodigiosa ma intermittente d'uno spirito che conflagrava a sbalzi, e prorompeva poi come la lava; e, inerte, pareva non avesse né pensieri né volontà di pensare, e non badasse a nessuno dei discorsi che si facevano intorno a lui; chè girava vagamente la semichiusa pupilla di cosa in cosa, come uno che abbia piuttosto volontà di dormire che d'operare; ma in realtà ascoltando tutto, e avvicinando le idee estreme che tumultuavano in quella sala nel cicaleccio di tante persone, e di ciascuna idea che gli paresse non rigettabile facendo base alla feconda generazione di tutte le idee conseguenti, colla prontezza d'una facoltà induttiva prodigiosa". ${ }^{233}$

E diverso anche da quello che Franco Venturi rappresenta: "Era ormai diventato un alto funzionario e la sua carriera si chiudeva ogni anno di più in una grigia e spenta normalità". ${ }^{234}$

La consultazione degli Atti di Governo, oggi così comodamente consultabili dallo studioso grazie alla grossa fatica ed alla fine intelligenza di chi ha ideato, voluto e realizzato l'intrapresa (Beccaria, sono sicurissimo, avrebbe proposto la concessione di un premio) mostrano un Beccaria che aveva piuttosto volontà d'operare che di dormire, per

233 G. Rovani, Cento anni, Milano 1934, I, p. 390.

234 F. Venturi, Beccaria, Cesare, Dizionario Biografico degli Italiani, VII, Roma 1965 , pp. 458-469, p. 467, in particolare. Ancor più severo, quasi impietoso, il giudizio già espresso dal Valsecchi: "Il Beccaria funzionario non vale certo il Beccaria pensatore; non escono dalla sua penna che insignificanti relazioni burocratiche, dalla sua attività, che provvedimenti di ordinaria amministrazione: spirito pigro e passivo, si adatta facilmente ad obbedire" [Valsecchi, 1934, p. 201]. Più appensato e maggiormente condivisibile, soprattutto perché fondato sullo studio di documenti allora inediti, è il giudizio espresso da Alberto Errera nell'adunanza 6 aprile 1876 dell'Istituto Lombardo: "Abituati a considerare il Beccaria poco adatto alla vita pratica, e protezionista per quanto si riferisce allo scambio internazionale, noi lo scopriremo, colla lettura di questi scritti, uomo assai valente nelle cose burocratiche, avveduto nelle questioni industriali e nemico di monopolj” [Errera, 1878, p. 161]. Sottolineava inoltre la superiorità di Beccaria rispetto a Carli e Pietro Verri "pella iniziativa delle riforme pratiche, pella stupenda inchiesta intorno a Como [del 1787, che si legge alle pp. 215-222, v. retro, note $23,135]$, per le proposte di vera utilità al commercio, alle industrie [...]" (ibid., p. 168). E ribadiva questo pensiero: "[Beccaria] sopravanzava perciò anche gli illustri Pompeo Neri, L. Ricci e lo stesso Pietro Verri, al quale è da meno come pensatore, mentre lo vince come impiegato e riformatore, scrivendo inoltre tali cose che anche oggidì potrebbero essere utilmente meditate nelle nostre pubbliche Amministrazioni” (ibid., p. 170). 
ripetere le parole di Giuseppe Rovani. ${ }^{235}$ Un Beccaria in azione fondamentalmente coerente con le idee espresse e con quanto scritto a tavolino: se mai, non già cedimenti vistosi, révirements, ma adattamento delle idee e dei principi alla realtà nella quale debbono operare, ispirando comportamenti e decisioni.

Significativa, al riguardo, è una consulta stesa in materia di istituzione di nuovi mercati, ove si legge: "Secondo l'opinione di molti valent'uomini - e Beccaria ben avrebbe potuto annoverare anche se stesso tra costoro, ${ }^{236}$ - il migliore di tutti li sistemi annonari sarebbe quello di una libertà assoluta: niente perciò di più semplice ad imaginare e proporre, e pure a quanti libri e a quante discussioni ha dato luogo un tale progetto". Ma se facile era "imaginare e proporre", non altrettanto lo era trovare "la via di combinare ad un tempo la facilità per invitare l'affluenza e la cautela per assicurare la pubblica indennità, alla quale difficoltà se ne aggiungeva un'altra suggerita dalle attuali circostanze del vegliante sistema”. La facilità poteva inoltre essere infranta dal "dovere di chi serve di sottomettere la propria opinione ai sovrani voleri [...]". E questo era il caso: "Se poi li mercati da stabilirsi esigevano qualche sorte di coattiva per lo stabilimento di essi, non sapevasi ciò combinare colla facilitazione e maggiore libertà di commercio, che la materna provvidenza di Sua Maestà ha voluto prescrivere e nel Piano e nelle posteriori sue iussioni". ${ }^{237}$ Si pensi anche a Beccaria alle prese con le complicate vicende legate alla libertà di commercio del grano, ovvero alla libera panificazione, vicende che qui, per ragioni di tempi, non ho potuto toccare, se non per brevi cenni [Capra, 1998, pp. 218 220; Id., 2000, pp. 69-71]..$^{238}$

Beccaria ci lascia dunque idee, concezioni, principi grandi nel

235 Pienamente condivisibile è il giudizio che suona: "Il divario fra il centinaio di documenti noti sino a ieri e gli oltre seimila che qui verranno via via trascritti e illustrati dà la misura di una grande fatica - seria, costruttiva, utile - che sgombrerà definitivamente l'orizzonte culturale dall'immagine di un Beccaria indolente, disamorato, per restituirci quello di un economista riformatore che quotidianamente riflette e agisce al servizio di uno Stato (che era pur la sua patria) e del progresso civile" (Firpo, 2009 (1987), p. 1086). E sono, queste, le parole conclusive di Canetta, 1990, p. 475.

236 Cfr., retro, p. 298.

237 BeCCARIA, Atti di Governo, VII [nt. 139], doc. 492, 16 luglio 1779, pp. 121 137 , in particolare, p. $125 \mathrm{~s}$.

238 Cfr., retro, pp. 373-378 (commercio del grano), 381-386 (panificazione). 
penale e nell'economia e, al contempo, la testimonianza di un'attività concreta straordinariamente intensa. Un Beccaria che, di certo, Giovanni Carmignani, penalista toscano dell'Ottocento, non avrebbe ascritto a quella categoria di giuristi configurati, con tono e intento spregiativi, come coloro "che occupano la bigoncia a suon di concetti e frasi”. 239

Una categoria alla quale Beccaria dimostrò di non appartenere con la sua costante attività di pubblico amministratore, in piena coerenza, ancora una volta ed anche sotto questo profilo, con quanto aveva scritto a tavolino: "Finalmente non piccolo vantaggio può arrecare lo studio d'una scienza non rinchiusa nella solitudine d'un gabinetto, non versante intorno ad oggetti remoti dall'uso promiscuo della vita, ma della quale tutti i circoli e le radunanze risuonano, e gli avvenimenti giornalieri ci richiamano a continue applicazioni [...]" ${ }^{240}$

In effetti, anche le poche carte (voti, relazioni, consulte, appunti su pratiche, lettere...) qui considerate rappresentano un Beccaria di certo non rinchiuso "nella solitudine d'un gabinetto", tutto dedito, anzi, a questioni ed affari ben immersi "nell'uso promiscuo della vita", attinenti a materie che, per importanza, varietà e complessità, sembrano lasciarlo talora sgomento, determinando in lui la sensazione dell'inadeguatezza ad affrontarle e risolverle degnamente, in modo da corrispondere alle aspettative di Sua Maestà, e utilmente, in vista del conseguimento di quel "bene pubblico", ch'è stato, peraltro, definito "mitico" [Mozzarelli, 1975, p. 255].

Chiare, al riguardo, sono le parole vergate nel luglio 1791, quando erano ormai avviati i lavori del complesso apparato messo a punto da Leopoldo II per la riforma del sistema criminale e della procedura civile in Lombardia. ${ }^{241}$ Qualora si fosse voluto attribuirgli la funzione di

239 G. Carmignani, Lettera a Francesco Forti, gennaio 1830. Cito da Geri, 2003, p. 285 s. e nota 56.

240 BECCARIA, Prolusione [nt. 5], p. 177: “[...] onde gioverà sempre il guardarsi per interno convincimento, e per quella luce tranquilla e chiara che le scienze solidamente studiate c'infondono, sia dai venerati pregiudizi che per domestica tradizione ci vengono tramandati, sia da quell'abituale querulità e malcontentezza, che non cessa in ogni tempo ed in ogni luogo di esser soffiata sulla diffidente e docile ignoranza".

241 Naufragati i lavori della Commissione istituita da Giuseppe II il 3 ottobre 1787 per l'adattamento del Codice penale agli usi ed alle circostanze della terra di Lombardia, Leopoldo nominò (17 febbraio 1791) una Giunta generale per l'“emendazione del sistema 
riferire nella Giunta civile, in nome della Conferenza governativa, "le questioni giurisdizionali per il rapporto che potessero avere” con il civile, vale a dire le questioni di dubbia competenza, "un aggravio altresì ne risulterebbe per il Consigliere Marchese Beccaria non compatibile colle altre sue occupazioni. Trovasi egli occupato a due sessioni del Magistrato ogni settimana; a due nella suddetta Giunta criminale; ad una per la Commissione Ecclesiastica, di cui pure è individuo per disposizione di Sua Maestà; e tutto ciò in aggiunta alle occupazioni di un Dipartimento ch'è de' più affollati”. ${ }^{242}$

Se affollato era il Dipartimento secondo, ${ }^{243}$ non di meno lo era il Dipartimento terzo, del quale Beccaria era Presidente nel 1787, quando provvide a stendere, su richiesta di Wilczeck, una relazione - definita da Alberto Errera "importantissima monografia" [Errera, 1878, p. 164] - volta ad illustrare l'attività svolta dal Dipartimento stesso. In essa ricorrono anche queste parole: "La molteplicità e la diversità degli oggetti che formano la materia di mille duecento cinquanta esibiti, intorno ai quali si è indefessamente occupato il Dipartimento Terzo dal primo maggio a tutto dicembre 1786, è tale che riesce dificile, nella strettezza del tempo e nella necessità di tener dietro al rapido corso degli affari giornaglieri, soddisfare pienamente alle superiori ricerche. Ciò nonostante, scorrendo le classi generali ${ }^{24}$ a cui hanno avuto rap-

giudiziario". Risultando eccessivamente gravoso il compito di provvedere alla riforma del penale e del civile, il 16 giugno 1791 venne istituita una Giunta separata e speciale incaricata della riforma del sistema criminale [Cavanna, 1975, pp. 60-85]. Delle diverse Commissioni aveva fatto e faceva parte Cesare Beccaria. Accanto alle Giunte giudiziarie civile e penale era prevista anche una Commissione giudiziario-politica, sulla cui funzione, volta a comporre i casi di "dubbio o controversia di giurisdizione", Beccaria ebbe ad esprimersi favorevolmente. Cfr., BECCARIA, Atti di Governo, XIII [nt. 46], doc. 4165, 25 luglio 1791, p. 306 s.; BeCCARIA, Atti di Governo, XII [nt. 62], doc. 3806, [nt. 179], p. 486.

242 BeCCARIA, Atti di Governo, XIII [nt. 46], doc. 4165, 25 luglio 1791, p. 305 s. In precedenza, al tempo del Magistrato Camerale, l'assenza, giustificata o no che fosse, dei due consiglieri relatori, faceva ricadere la molteplice attività dell'ufficio annonario sul solo Beccaria, "il quale -rilevava il Carli- tutto ciò sia dotato della maggiore attività e della migliore intenzione del mondo, non può però superare l'impossibilità invincibile di supplire alle incombenze alle quali tre bastar possono appena”. Cito da Carera, 1990, p. 430.

243 Alle cure del quale Beccaria era stato assegnato alla fine del 1789 [Canetta, 2009, p. 1052].

244 "Le classi generali a cui si riducono le materie del Dipartimento Terzo sono commercio, manifatture, arti, università e camere mercantili, sovvenzioni del Fondo 
porto i succenati esibiti, m'ingegnerò di dare un'idea di ciò che si è fatto di più importante, dei principii con cui sono state regolate le operazioni analoghe, senza trascurare tutto ciò che si ha in vista o si potrebbe avere riguardo alle inspezioni affidate al Dipartimento". ${ }^{245}$

Sono parole che ci consentono di cogliere, di vedere al vivo Beccaria intento nell'esercizio delle sue funzioni: quotidianamente alle prese con molti affari da "disimbarazzare", affari in genere complessi e complicati per motivi diversi, ${ }^{246}$ intricati, ${ }^{247}$ comunque sempre importan$\mathrm{ti}^{2},{ }^{248}$ che richiedevano tempo per essere utilmente affrontati e risolti, un tempo che quasi sempre mancava o scarseggiava. ${ }^{249} \mathrm{E}$ Beccaria sovente

del Commercio, somministrazioni in moneta di rame, pesi e misure, fiere e mercati, miniere, veterinaria, Società Patriotica, annona e vettovaglie, cacce e popolazione". BeCCARIA, Atti di Governo, IX [nt. 19], doc. 1689, Attività del Dipartimento III nel 1786 (relazione, 31 marzo 1787), p. 224. Alcune di queste materie, come annona e caccia, vennero mantenute nell'ambito di competenza del Dipartimento secondo ed altre gli vennero attribuite, tra le quali polizia, Ergastolo di Pizzighettone, Casa di correzione per ricordare quelle che gli atti di Governo beccariani qui considerati ci hanno consentito di conoscere [Capra, 1984, pp. 516-518; Canetta, 1990, pp. 469472; Francioni, 2000, p. 5].

245 Ibid., p. 223.

246 Talora si trattava di una "materia [quella della caccia] ch'era per me del tutto nuova e inviluppata con molti rapporti del vecchio sistema, ora del tutto cambiato" (BeCCARIA, Atti di Governo, XI [nt. 9], doc. 2851, 19 gennaio 1789, p. 85). Altrove Beccaria si riferisce "alla novità del sistema, alla difficoltà di ritrovare gli anteatti esistenti prima del'erezione del Consiglio [...]" (BECCARIA, Atti di Governo, IX [nt. 19], doc. 1689, Attività del Dipartimento III nel 1786 (relazione, 31 marzo 1787), p. 223). "Ecco quanto io ho creduto, per ora, di dover suggerire in un affare [quello della riforma dei pesi e delle misure] che diventa complicato ogni volta che chi ha l'onore d'impiegarvisi abbia zelo e premura di volerne prevedere tutte le particolarità, e di non fermarsi solo sulle mire generali [...]" (BECCARIA, Atti di Governo, VII [nt. 139], doc. 547, 25 gennaio 1780, pp. 252-310, sul punto, p. 280).

247 "Mi lusingo di aver dati quei schiarimenti che per ora posso dare su questa assai intricata materia [quella della panificazione] [...]" (BECCARIA, Atti di Governo, XI [nt. 9], doc. 3048, 2 giugno 1789, p. 351.

248 Per un esempio, BeCCARIA, Atti di Governo, VI [nt. 25], doc. 4, 9 agosto 1771, p. 27: "Perciò, in mezzo alla molteplicità delli affari importanti, che tutti insieme esigevano l'assiduità e la cura la più minuta e sollecita di questo nuovo Dicastero di Vostra Maestà, ebbero sempre li soggetti che lo componevano rivolti i pensieri anche a questo principalissimo ogetto [quello della rifusione delle monete]".

249 BeCCARIA, Atti di Governo, IX [nt. 19], doc. 1846, Codice generale (relazione, 
palesa il timore di essere risultato impari alle aspettative sovrane, denuncia la debolezza del suo talento, ${ }^{250}$ la tenuità dei suoi lumi, ${ }^{251} \mathrm{ma}$, in pari tempo, è fieramente conscio di aver profuso ogni sforzo, in modo costante ed assiduo, nel far fronte ai diversi impegni "per quanto ce lo permettevano le nostre forze, sempre inferiori a quel puro zelo" - per il quale egli "è più volte lodato sia nelle consulte del magistrato sia dal

[giugno 1787]) Brevi riflessioni [nt. 124], p. 477: "Ciò farò nel miglior modo che mi sarà permesso dalle ben note mie circostanze, dalla brevità del tempo e dalla multiplicità degli affari di ufficio, in via di semplici annotazioni”; BECCARIA, Atti di Governo, VII [nt. 139], doc. 547, 25 gennaio 1780, p. 253: "Queste ragioni hanno contribuito a ritardare la speditezza del lavoro oltre quanto possa avervi avuta parte l'assiduità mia al Tribunale, il disimpegno delle altre numerose mie incumbenze, oltre il tempo consumato nel raccogliere le necessarie notizie e quello che si è speso nel rettificare le proprie idee sul fatto". Ed ancora: "La maggior tardanza, dunque, che potrebbe imputarsi al relatore" era invero scusabile dal momento che il tempo in cui egli doveva occuparsi dell'istituzione di nuovi mercati era anche il "tempo in cui, oltre la spedizione degli affari correnti, il Relatore doveva occuparsi $[\ldots]$ negli affari di monete che allora più che mai si affollavano per l'imminente sistema monetario [...]" (BECCARIA, Atti di Governo, VII [nt. 139], doc. 492, 16 luglio 1779, pp. 121-137, sul punto, p. 124 s.). Incombente era inoltre la necessità di riformare pesi e misure. Agli incalzanti Kaunitz e Firmian, Beccaria, convinto d'avere eseguito il proprio compito senza eccessivo ritardo, spiegava come avesse "impiegato il tempo necessario a maturare e placidamente eseguire un'operazione che direttamente si oppone alle radicate consuetudini del popolo, dalle quali è malagevole lo svolgerlo senza riclami ed inconvenienti, e ciò avendo procurato di fare senza arenamento degli affari d'Annona, che anche nell'attuale sistema di facilità non lasciano di essere moltissimi [...]". Ed occorreva non dimenticare che l'anno precedente aveva "subordinato due lunghe relazioni annonarie [quelle del 28 aprile e del 15 settembre 1781(v. retro, note 139, 197-203, 220.]", nel mentre "[...] non è mai stata interrotta la molteplice spedizione degli affari di Annona [...]": si trattava di una fatica che, "benché sia materiale, esige però tutta quella attenzione che è indispensabile a prevenire gli abusi [...]" (BECCARIA, Atti di Governo, VII [nt. 139], doc. 716, 4 aprile 1782, pp. 661-664) [Canetta, 1990, p. 473 s.].

250 BeCCARIA, Atti di Governo, IX [nt. 19], doc. 1846, Codice generale (relazione, [giugno 1787]) Brevi riflessioni [nt. 124], p. 490: "Del resto, io la supplico di perdonare gli errori e le ommissioni contenute in questo scritto, attribuendole alla debolezza del mio talento, al non essere da lungo tempo versato nelle criminali materie, delle quali mi è sempre mancata la pratica, e alle attuali mie circostanze di ufficio e domestiche, ben note all'Eccellenza Vostra [Wilczeck]".

251 Beccaria coglie un motivo di difficoltà "nella necessità in cui mi trovo di dover far camminar di fronte oggetti molto disparati tra di loro ed a cui non lascio di accudire con quella sollecitudine che porta il mio dovere e con quella ponderatezza che è proporzionata all'importanza degli oggetti e molto più alla tenuità de' miei lumi" 
Cancelliere, non solo per l'annona" [Carera, 1990, p. 440 e nt. 127] "che ci anima nel glorioso servigio di Vostra Maestà [...]". ${ }^{252}$

Un Beccaria che mostra inoltre la chiara e piena consapevolezza della dignità della propria funzione di alto magistrato, fermo nel salvaguardare le prerogative di tale funzione. Lo dimostra un piccolo, piccolissimo episodio attinente all'argomento premio, che ora, essendomi, in questa relazione, su siffatto argomento un poco indugiato, ricordo. Premi erano previsti "pei liberatori dei sommersi", cioè per coloro che avessero soccorso gli annegati. In merito, una consulta di Beccaria del 3 febbraio 1794 c'informa che intorno alla concessione del premio indicato era sorto un conflitto di competenza tra la Congregazione Municipale di Milano e il Magistrato Politico Camerale circa l'interpretazione e applicazione del $\$ 154$ del Piano del Magistrato stesso. Della controversia era stata investita la Reale Conferenza Governativa. Ciò che mi pare qui utile rilevare è come Beccaria si lagnasse perché "il sistema stabilito da S. Maestà [sembra] esigere, che esista una gerarchia politica, giusta la quale un Dicastero inferiore [i.e. la Congregazione Municipale] deve attenersi, nel rappresentare le sue occorrenze al Dicastero superiore, alle espressioni convenienti alla stabilita subordinazione $[\ldots] " .253$

In conclusione, lascio, ancora una volta, la parola a Beccaria, che così terminava la relazione intorno all'attività svolta dal Dipartimento III nei primi dieci mesi della sua attività: "Ecco tutto ciò che di più importante è passato nei dieci mesi nei quali io ho l'onore di servire Sua

(BeCCARIA, Atti di Governo, XI [nt. 9], doc. 3048, 2 giugno 1789, p. 351). Ripreso dalla Corte per la sua scarsa sollecitudine, Beccaria si difendeva: "Su questa apparente dilazione però si prega considerare che, oltre all'affollamento degli affari che esigono pronta spedizione [il Relatore] ha dovuto consumare qualche tempo per pensare e risolvere quello che un più pronto ingegno avrebbe forse più presto pensato $\mathrm{e}$ risolto. Sono semplici, è vero, e poche le avvertenze che si sono proposte in quella consulta, ma moltissime, per non dire il più delle volte, non si arriva a questa semplicità se non passando a traverso di una quantità di pensieri complicati, che si devono rigettare l'uno dopo l'altro per i dubbi e le difficoltà che si presentano alla immaginazione" (BECCARIA, Atti di Governo, VII [nt. 139], doc. 492, 16 luglio 1779, pp. 125 137 , in particolare, p. 123).

252 BeCCARIA, Atti di Governo, VI [nt. 25], doc. 4, 9 agosto 1771, p. 65.

253 BeCCARIA, Atti di Governo, XVI-I [nt. 66], doc. 5813, 3 febbraio 1794, pp. 72-82, sul punto, p. 82 . 
Maestà nel Regio Imperial Consiglio, lo che facendo in esecuzione dei superiori comandi, ho procurato di esporre non solamente le viste che si sono avute, ma ancora quelle che secondo la debolezza de' miei talenti si potrebbero avere nelle materie che particolarmente mi concernano, ciò che spero mi sarà perdonato in favore di quel puro zelo che mi anima nel reale e pubblico serviggio, per cui mi lusingo anche di un benigno compatimento se, per la molteplicità delle cose che ho creduto dover subordinare in questa occasione, ho prolungato l'estesa di questa mia Relazione, approffittando dei pochi ritagli di tempo che mi lasciava l'incessante spedizione degli affari giornaglieri". ${ }^{254}$

254 BeCCARIA, Atti di Governo, IX [nt. 19], doc. 1689, Attività del Dipartimento III nel 1786 (relazione, 31 marzo 1787), p. 267. 


\section{LETTERATURA CITATA NEL TESTO}

Apih, 1973 E. APIH, Rinnovamento e illuminismo nel '700 italiano. La formazione culturale di Gian Rinaldo Carli, Trieste.

Barucci, 1965 P. BARUCCI, Il pensiero economico di Melchiorre Gioia, Milano.

Brambilla, 2003 E. BRAMBILLA, La polizia dei tribunali ecclesiastici e le riforme della giustizia penale, in Corpi armati e ordine pubblico in Italia (XVI-XIX sec.), Soveria Mannelli, pp. 73-110.

Burgio, 2000 A. BuRgio, L'idea di eguaglianza tra diritto e politica nel Dei delitti e delle pene, in Cesare Beccaria. La pratica dei Lumi, IV giornata Luigi Firpo, Atti del Convegno 4 marzo 1977, a cura di V. Ferrone e G. FranCIOnI, Firenze, pp. 79-98.

Caizzi, 1957 B. CAIzzI, Storia del setificio comasco, I. L'economia, Centro lariano per gli studi economici. Raccolta di saggi e ricerche dirette da G. MigLio, Como.

Caizzi 1968 B. CAIZZI, Industria, commercio e banca in Lombardia nel XVIII secolo, Milano.

Canetta, 1973 R. CANETTA, Questioni agricole milanesi in alcuni scritti inediti di Cesare Beccaria, in AA. VV., Aspetti di vita agricola lombarda (secoli XVI-XIX) a cura di M. RomanI, Contributi dell'Istituto di storia economica e sociale, I, Milano, pp. 3-183.

Canetta, 1990 R. CANETTA, L'attività di governo di Beccaria alla luce di recenti indagini: primi risultati, problemi e prospettive, in Cesare Beccaria tra Milano e l'Europa.

Prolusioni di S. RomagnOLI e G.D. PisaPIA, Milano, Roma-Bari, 1990, pp. 468-478.

Canetta, 2009 (1987), R. CAnetta, Nota al testo, in C. BeCCARIA, Atti di Governo (serie XI: gennaio-dicembre 1794), a cura di R. CANETTA, II (settembre-dicembre 1794), vol. XVI dell'Edizione Nazionale delle Opere diretta da L. FIRPO e G. FRANCIONI, Milano, pp. 1045-1080.

Capra, 1984 C. CAPra, Il Settecento, in D. Sella-C. CaPra, Il Ducato di Milano (15351786), Storia d'Italia diretta da G. GALASSO, volume undicesimo, Torino.

Capra, 1985 C. CAPRA, Il "Mosè della Lombardia": la missione di Carlo Antonio Martini a Milano, 1785-1786, in Il Trentino nel Settecento fra Sacro Romano Impero e antichi stati italiani a cura di C. MozzARELLI e G. Olmi, Annali dell'Istituto storico italo-germanico. Quaderni 17, Bologna, pp. 323-351.

Capra, 1998 C. CAPRA, Gli intellettuali e il potere: i casi di Beccaria e di Verri, in L'età dei Lumi. Saggi sulla cultura settecentesca, a cura di A. SANTUCCI, Bologna, pp. 211-230.

Capra, 2000 C. CAPRA, Il gruppo del «Caffè» e le riforme, in Cesare Beccaria. La pratica dei Lumi, IV giornata Luigi Firpo, Atti del Convegno 4 marzo 1977, a cura di V. Ferrone e G. Francioni, Firenze, pp. 63-78.

Capra, 2002 C. CAPRA, I progressi della ragione. Vita di Pietro Verri, Bologna. 
Carera, 1990 A. CARERA, Cesare Beccaria, magistrato in "provincia d'annona", in Cesare Beccaria tra Milano e l'Europa. Prolusioni di S. Romagnoli e G.D. PISAPIA, Milano, Roma-Bari, 1990, pp. 425-467.

Cassi 1999 A.A. CASSI, Il "bravo funzionario" asburgico tra Absolutismus e Aufklârung. Il pensiero e l'opera di Karl Anton von Martini (1726-1800), Milano.

Cattaneo, 1997 M.A. CATTANEO, Les fondaments philosophiques de la fonction de la peine chez Beccaria, in Beccaria et la culture juridique des Lumières. Etudes historiques éditées et présentées par M. PORRET, Genève, pp. 83-88.

Cavanna,1975 A. CAVANNA, La codificazione penale in Italia. Le origini lombarde, Milano.

Cavanna, 2005 A. Cavanna, Storia del diritto moderno in Europa. Le fonti e il pensiero giuridico, 2, Milano.

Chiappetti, 1973 A. ChiappetTi, L'attività di polizia, Padova.

Cuccia, 1971 S. CUCCIA, La Lombardia alla fine dell'ancien régime. Ricerche sulla situazione amministrativa e giudiziaria, Pubblicazioni della Facoltà di Lettere e Filosofia dell'Università di Milano LVIII, Firenze.

Di Renzo Villata, 2000 M.G. Di Renzo Villata, Beccaria e gli altri tra ieri e oggi (Alcune riflessioni a margine della relazione Zagrebelsky), in Cesare Beccaria. La pratica dei Lumi, IV giornata Luigi Firpo, Atti del Convegno 4 marzo 1977, a cura di V. Ferrone e G. Francioni, Firenze, pp. 23-47.

Di Simone, 2000 M.R. Di SimONE, Riflessioni sulle fonti e la fortuna di Cesare Beccaria, in Cesare Beccaria. La pratica dei Lumi, IV giornata Luigi Firpo, Atti del Convegno 4 marzo 1977, a cura di V. FERRONE e G. FranCIONI, Firenze, pp. 49-61.

Errera, 1878 A. ERRERA, Una nuova pagina della vita di Cesare Beccaria. Memoria corredata da documenti inediti, tratti dagli archivj governativi di Milano, memoria letta nell'adunanza del 6 aprile 1976, in Memorie del Reale Istituto Lombardo di Scienze e Lettere, s. III, XIII (fasc. 4, 1878), pp. 161-222.

Firpo, 2009 (1987) L. FIRPO, Bibliografia, in C. BECCARIA, Atti di Governo (serie XI: gennaio-dicembre 1794), a cura di R. CANETTA, II (settembre-dicembre 1794), vol. XVI dell'Edizione Nazionale delle Opere diretta da L. FIRPO e G. FRANCIONI, Milano 2009, pp. 1081-1110.

Francioni, 2000 G. Francioni, Notizie dal cantiere dell'Edizione Nazionale beccariana, in Cesare Beccaria. La pratica dei Lumi, IV giornata Luigi Firpo, Atti del Convegno 4 marzo 1977, a cura di V. FERRONE e G. FranCIONI, Firenze, pp. 1-12.

Frumento, 1963 A. FRUMENTO, Imprese lombarde nella storia della siderurgia italiana vol. II Il ferro milanese tra il 1450 e il 1796, Milano.

Garlati, 2006 L. Garlati, La parola alla difesa. Profili penalistici nelle allegationes lombarde tra Sette e Ottocento, in L'arte del difendere. Allegazioni avvocati e storie di vita a Milano tra Sette Ottocento a cura e con un saggio introduttivo di M.G. DI RENZO Villata, Milano, pp. 435-525.

Geri, 2003 M.P. GERI, L'epistolario di Carmignani tra letture e incontri professionali e culturali, in Giovanni Carmignani (1768-1847). Maestro di scienze criminali e pratico del foro, sulle soglie del Diritto Penale contemporaneo a cura di M. MONTORZI, Pisa, pp. 273-301. 
Gorla, 1981 G. GoRLA, I tribunali supremi degli Stati Italiani, fra i secc. XVI-XIX, quali fattori della unificazione del diritto nello Stato e della sua uniformazione fra Stati (Disegno storico comparativo) (1977), in ID., Diritto comparato e diritto comune europeo, Milano, pp. 543-617.

Grab, 1982 A.I. GRAB, Le riforme annonarie nello Stato di Milano (1765-1786), in Economia, istituzioni, cultura in Lombardia nell' età di Maria Teresa, III Istituzioni e società a cura di A. De MADDALEnA E. Rotelli G. BARBARIsI, Bologna, pp. 399-442.

Grab, 1986 A.I. GRAB, La politica del pane. Le riforme annonarie in Lombardia nell'età Teresiana e Giuseppina, Milano.

Liva, 1990 A. Liva, Carcere e diritto a Milano nell'età delle riforme: la Casa di correzione e l'Ergastolo di Milano da Maria Teresa a Giuseppe II, in La "Leopoldina". Criminalità e giustizia criminale nelle riforme del '700 europeo. Ricerche coordinate da L. BERLINGUER 11 Le politiche criminali nel XVIII secolo a cura di L. Berlinguer e F. Colao, Milano, pp. 63-142.

Massetto, 1994a G.P. MASSETTO, Aspetti della prassi penalistica lombarda nell'età delle riforme: il ruolo del Senato milanese (1981), in ID., Saggi di storia del diritto penale lombardo (Secc. XVI-XVIII), Milano, pp. 331-424.

Massetto, 1994b G.P. MASSETTO, Economia e pena nell'opera del Beccaria (1990), in ID., Saggi di storia del diritto penale lombardo (Secc. XVI-XVIII), Milano, pp. 495-542.

Mozzarelli, 1975 C. Mozzarelli, "Pubblico bene" e Stato alla fine dell'ancien régime. Efficienza amministrativa e modello di sviluppo nella esperienza giuseppina, in Jus Rivista di scienze giuridiche, n.s., XXII (fasc. 2, 1975).

Mozzarelli, 1990 C. MozZarelli, Le Intendenze politiche della Lombardia austriaca (1786-1791), in L'organizzazione dello Stato al tramonto dell'Antico regime a cura di R. De Lorenzo, Napoli, pp. 61-118.

Neppi Modona, 1990 G. NEPPI ModOnA, L'utile sociale nella concezione penalistica di Cesare Beccaria, in Cesare Beccaria and Modern Criminal Policy. Milan (Italy), Castello Sforzesco December 15th-17th, 1988, Milano, pp. 77-97.

Nutini, 1989 S. Nutini, Vincenzo Cuoco a Milano (1800-1806). Inediti e rari, Roma.

Pagano, 2011 E. Pagano, Giustizia e crimine nel Mantovano del tardo Settecento, in Archivio Storico Lombardo, CXXXVII (2011), pp. 163-211.

Pansini, 1988 G. PANSINI, La riforma del sistema criminale nell'età di Pietro Leopoldo, in La "Leopoldina". Criminalità e giustizia criminale nelle riforme del Settecento europeo. Ricerca coordinata da L. BERLINGUER, vol. I, Preprint, Milano.

Pasta, 1997 R. PASTA, Dei delitti e delle pene et sa fortune en Italie: les milieux juridiques et la lecture des «philosophes», in Beccaria et la culture juridique des Lumières. Etudes historiques éditées et présentées par M. PORRET, Genève, pp. 119-148.

Pisani, 1998 M. PISANI, Attualità di Cesare Beccaria, Milano, III. Beccaria e il diritto premiale, pp. 41-60.

Pisani, 2011 M. PISANI, Cesare Beccaria e il principio di umanità, in Rivista italiana di diritto e procedura penale, LIV 2 (2011), pp. 407-413.

Porta e Scazzieri, 1996 P.L. PorTA e R. SCAzzIERI, Concorrenza e società civile, in Alle 
origini del pensiero economico in Italia, 2. Economia e istituzioni. Il paradigma lombardo tra $i$ secoli XVIII e XIX a cura di A. QuADrIO CuRZIO, Bologna, pp. 15-58.

Provin, 1990 G. Provin, Una riforma per la Lombardia dei Lumi. Tradizione e novità nella "Norma interinale del processo criminale", Milano.

Quadrio Curzio, 2002 A. QuADRIO CURZIO, Il "paradigma economico lombardo" è riformatore, tecnologico ed europeista, in C. Cattaneo, Scritti sulla Lombardia, vol. IV de La Lombardia di Carlo Cattaneo, Milano, pp. XI-XXIV.

Romani, M. 1977 M. RomanI, L'economia milanese nel Settecento (1958), ora in Aspetti e problemi di storia economica lombarda nei secoli XVIII e XIX Scritti riediti in memoria, Milano 1977, pp. 122-206.

Romani, R. 1990 R. Romani, Un popolo da disciplinare: l'economia politica di Melchiorre Gioia come sapere amministrativo, in Melchiorre Gioja 1767-1829. Politica, società, economia tra riforme e restaurazione Atti del convegno di studi, Piacenza, 5-7 aprile 1990, Bollettino Storico Piacentino, LXXXV (1990), pp. 303-329.

Sbriccoli, 1997 M. SBRICCOLI, Beccaria ou l'avènement de l'ordre. Le philosophe, les juristes et l'émergence de la question pénale, in Beccaria et la culture juridique des Lumières. Etudes historiques éditées et présentées par M. PORRET, Genève, pp. 177-187.

Trezzi, 1979 L. TrezzI, L'azione dei governanti a favore dell'agricoltura dello Stato di Milano nella seconda metà del Settecento, in AA.VV., Questioni di storia agricola lombarda nei secoli XVIII-XIX Le condizioni dei contadini, le produzioni e l'azione pubblica a cura di S. ZANINELLI, Contributi dell'Istituto di storia economica e sociale, IV, Milano, pp. 219-343.

Trezzi, 1982 L. TrezzI, Un sostegno dell'attività manifatturiera dello Stato di Milano. Il fondo di commercio, l'avvio e le prime realizzazioni (1750-1774), in Economia, istituzioni, cultura in Lombardia nell'età di Maria Teresa, I Economia e società a cura di A. De Maddalena E. Rotelli G. Barbarisi, Bologna, pp. 175-190.

Valsecchi, 1934 F. VALSECCHI, L'assolutismo illuminato in Austria e in Lombardia, vol. secondo La Lombardia, Bologna.

Vassalli, 1990 G. VASSALlI, Spunti di politica criminale in Cesare Beccaria, in Cesare Beccaria and Modern Criminal Policy. Milan (Italy), Castello Sforzesco December $15^{\text {th }}-17^{\text {th }}, 1988$, Milano, pp. 23-35. 


\section{NOTA BIBLIOGRAFICA}

G. PECCHIO, Storia della economia pubblica in Italia ossia epilogo critico degli economisti italiani, preceduto da un'introduzione, Lugano, 1849; C. CANTù, Beccaria e il diritto penale. Saggio, Firenze 1862; L. CossA, Sulle prime cattedre di economia politica in Italia, in Rendiconti del Reale Istituto Lombardo di Scienze e Lettere, VI (1873), pp. 225-272; ID., Guida allo studio dell'economia politica, Milano, 1876; E. VERGA, Cesare Beccaria e la disciplina degli operai, in La Lettura, III, (1903), Milano, pp. 320322; A. VISCONTI, La pubblica amministrazione nello Stato milanese durante il predominio straniero. Saggio di storia del diritto amministrativo (1541-1796), Roma 1913; ID., Note sulla condizione degli operai agli albori dell'industria libera in Lombardia nel secolo XVIII, in Città di Milano. Bollettino municipale mensile di cronaca amministrativa e di statistica, XXVIII (1922), pp. 413-417 (fasc. ottobre), 456-459 (fasc. novembre); A. Mauri, La dottrina economica di Pietro Verri, Milano, 1924; F. LuZzATto, Cesare Beccaria e la politica agraria, in La riforma sociale 34 (1927), pp. 502-517; ID., La politica agraria nelle opere di Melchiorre Gioia, Piacenza, 1929; L. DAL PANE, La questione del commercio dei grani nel Settecento in Italia, vol. I, Milano 1932; C.A. Vianello, Il Settecento milanese, Milano, 1934; ID., (a cura di), Saggi inediti di Gian Rinaldo Carli: sull'economia pubblica dello stato di Milano, con prefazione di G. Galbiati, Firenze 1938; ID., La vita e l'opera di Cesare Beccaria con scritti e documenti inediti, Milano 1938; L. EINAUDI, Corporazioni d'arti e mestieri, bilancio del commercio e moneta negli economisti milanesi della seconda metà del Settecento, in Rivista di storia economica V (1940), pp. 267-275; C.A. Vianello (a cura di), Considerazioni sull'annona nello Stato di Milano nel XVIII secolo, Milano 1940; ID., (a cura di), La riforma finanziaria nella Lombardia austriaca nel XVIII secolo, Milano 1940; ID., (a cura di), Economisti minori del Settecento lombardo, Milano, 1942; ID., Introduzione a Economisti minori del Settecento lombardo, Milano 1942; ID., (a cura di), Le consulte amministrative inedite di Cesare Beccaria, Milano, 1943; ID., La crisi di sviluppo dell'industria serica comasca da Maria Teresa a Napoleone, in Periodico storico comense V (1944), pp. 120-151; F. CATALANO, Aspetti della vita economico-sociale della Lombardia nel secolo XVIII, in Nuova rivista storica XXXVIII (1954), pp. 25 78; L. Dal Pane, Le riforme economiche del '700, in Rassegna storica toscana I (1955); M. Romani, L'agricoltura in Lombardia dal periodo delle riforme al 1859. Struttura, organizzazione sociale e tecnica, Milano 1957; L. DAL PANE, Storia del lavoro in Italia dagli inizi del secolo XVIII al 1815, Milano 1958; L. AmBROsOLI, La formazione di Carlo Cattaneo, illustrata da un'appendice di scritti inediti o dimenticati, Milano-Napoli, 1959; F. CORPACI, Il contrattualismo di Beccaria, in Rivista internazionale di filosofia del diritto XXXVI (1959), pp. 237-269; D. SEVERIN, Storia dell'industria serica comasca (XVIII-XIX secolo), Como 1960; P. BARUCCI, Il pensiero economico di Melchiorre Gioia, Milano, 1965; A. Frumento, Imprese lombarde nella storia 
della siderurgia italiana. II Il ferro lombardo tra il 1450 e il 1796, Milano 1963; S. ZANINELLI, L'industria del cotone in Lombardia dalla fine del Settecento alla unificazione del paese, Torino 1967; G. ZARONE, Etica e politica nell'utilitarismo di Cesare Beccaria, Napoli 1971; M.A. CATTANEO, La filosofia della pena nei secoli XVII e XVIII. Corso di Filosofia del diritto, Ferrara 1974; L. FACCINI, I lavoratori delle risaie fra '700 e '800, in Studi Storici 15 (1974), pp. 545-588; A. MoIOLI, L'economia italiana preunitaria. Lombardia (1700-1859) L'editoria milanese, volume primo e secondo, Saggio bibliografico di A. Moioli, in Contributi dell'Istituto di storia economica e sociale a cura di M. Romani, I-II, Milano 1974; A. CaVanna, La codificazione penale in Italia. Le origini lombarde, Milano 1975; R. CANETTA, L'irrigazione nella bassa pianura lombarda tra il Sette e l'Ottocento, in AA.VV., Le campagne lombarde tre Sette e Ottocento. Alcuni temi di ricerca a cura di M. RomanI, Contributi dell'Istituto di storia economica e sociale, III, Milano, 1976, pp. 67-140; L. FACCINI, L'economia risicola lombarda dagli inizi del XVIII secolo all'Unità, Milano, 1976; M. ROMANI, Beccaria economista (1966), in ID., Aspetti e problemi di storia economica lombarda nei secoli XVIII e XIX Scritti riediti in memoria, Milano 1977, pp. 431-442; C. CAPRA, Le magistrature finanziarie dello Stato di Milano 1737-1771, in Istituzioni e attività finanziarie milanesi dal XIV al XVIII secolo, Convegno di studi, Milano 20-22 ottobre 1977 (relazione provvisoria); C. MozzarelLI, Il Magistrato camerale nella Lombardia austriaca (17711786), ibid., pp. 399-414 (relazione provvisoria); A.J. GRAB, Le riforme dei tribunali civici milanesi dal 1771 al 1786, in Archivio Storico Lombardo CIII (1977), pp. 296332; M. SCAZzOSO, Tentativi di riforma burocratica nella Lombardia austriaca: il Supremo Consiglio di economia (1765-1771), in Archivio storico lombardo CIII (1977), p. 198-211; A. ForTi Messina, La "disciplina degli operai" in Lombardia dopo la soppressione delle corporazioni (1787-1796), in Società e storia, I (1978), pp. 481 500; L. PANZERI, L'industria del ferro in Valsassina alla fine del Settecento, in Il Risorgimento XXX (1978), pp. 14-54; C. CAPRA, Riforme finanziarie e mutamento istituzionale nello Stato di Milano: gli anni Sessanta del secolo XVIII, in Rivista storica italiana XCI (1979), pp. 313-368; C. CAPRA, Lo sviluppo delle riforme asburgiche nello Stato di Milano, in La dinamica statuale austriaca nel XVIII e XIX secolo, a cura di P.A. SCHIERA, Bologna 1981, pp. 161-187; Economia, istituzioni, cultura in Lombardia nell' età di Maria Teresa, I Economia e società a cura di A. DE MADDALENA E. Rotelli G. Barbarisi, Bologna 1982 (v. i saggi di E. Borruso, Carlo Antonio Vianello e le fonti dell'economia lombarda, pp. 51-58; R. CANETTA, Gli studi agronomici in Lombardia durante l'età teresiana, pp. 59-75; A. MoIOLI, Aspetti della produzione e del commercio della seta nello Stato di Milano durante la seconda metà del Settecento, pp. 151-173; S. ZANINELLI, Economia e società lombarda in epoca teresiana, pp. 351-361); V. Molla Losito, La Società Patriottica di Milano (1776-1796), in Economia, istituzioni, cultura in Lombardia nell'età di Maria Teresa, III Istituzioni e società a cura di A. De Maddalena E. Rotelli G. BARbarisi, Bologna 1982, pp.1039-1055; A. SCOTTI, Distribuzione, tipologia e scelte formali di alcuni edifici di "pubblica utilità" nella Milano del secondo Settecento, in Città e controllo sociale in Italia tra XVIII e XIX secolo a cura di E. SORI, Milano 1982, pp. 219-250; E. DeZZA, Il codice di procedura penale del regno Italico (1807). Storia di un decennio di elabora- 
zione legislativa, Padova, 1983, pp. 1-18; R. CANOSA-I. Colonnello, Storia del carcere in Italia dalla fine del '500 all'unità, Roma 1984; D. PARISI ACQUAVIVA, Il pensiero economico in Italia (1750-1860). Criteri definitori ed evoluzione storica, Milano 1985; Sul disordine delle monete a Milano nel Settecento. Tre saggi di Cesare Beccaria e di Pietro Verri. Introduzione A. QuAdrio CuRzIo e R. SCAZZIERI. Quadri storici M. Bortolotti e B. Cereghini, Milano, 1986; S. BAia Curioni, La ricchezza tra gelsi e riso, in L'Europa riconosciuta, Milano, 1987, pp. 197-227; A. CAPELLI, La buona compagnia. Utopia e realtà carceraria nell'Italia del Risorgimento, Milano 1988; C. MANGIO, La polizia toscana. Organizzazione e criteri d'intervento (1765-1808), in La "Leopoldina". Criminalità e giustizia criminale nelle riforme del '700 europeo. Ricerche coordinate da Luigi Berlinguer, Milano 1988; A. MACCHIORO, La "philosophia naturalis" gioiana dell'economia, in Politica, società, economia tra riforme e restaurazione. Atti del Convegno di studi, Piacenza 5-7 aprile 1990, in Bollettino Storico Piacentino, LXXXV (1990), pp. 269-302; R. RomAnI, Un popolo da disciplinare, l'economia politica di Melchiorre Gioia come sapere amministrativo, ibid., pp. 303-329; F. SOFIA, Melchiorre Gioia e la statistica, ibid., pp. 303-329; C. CAPRA-M.T. CISERANI, Criminalità e repressione della criminalità in Lombardia nell' età delle riforme: appunti per una ricerca, in La "Leopoldina". Criminalità e giustizia criminale nelle riforme del '700 europeo. Ricerche coordinate da L. BERLINGUER, 12: Criminalità e società in età moderna a cura di L. BerLINGUER e F. COLAO, Milano 1991, pp. 1-23; L. ANTONIELLI, La polizia nello Stato di Milano tra antico regime e età napoleonica. Appunti per una ricerca, in "Il Principato citeriore tra Ancien Régime e conquista francese: il mutamento di una realtà periferica del Regno di Napoli". Atti del Convegno di Salerno 14-16 maggio 1991 a cura di E. Granito, M. Schiavino, G. Foscari, Salerno, 1992, pp. 103-133; E. DEZZA, Tentativi di riforma del processo penale durante la prima Repubblica Cisalpina (1979), in ID., Saggi di storia del diritto penale moderno, Milano, 1992, pp. 69-157; G.P. MASSETTO, Note storico-giuridiche in tema di marchio (1880-1930), in I marchi di fabbrica a Milano 1868-1913 Arte, industria e diritto, Milano 1992; Le più belle pagine di Carlo Cattaneo scelte da G. SALVEMINI. Postfazione di L. CAFAGNA, Roma 1993; Alle origini del pensiero economico in Italia. 2 Economia e istituzioni. Il paradigma lombardo tra $i$ secoli XVIII e XIX, a cura di A. QuAdrio CuRZIO, Bologna, 1996; Mario PISANI, Beccaria e il diritto premiale, in Attualità di Cesare Beccaria, Milano, 1998, pp. 41-60; Temi e questioni di storia economica e sociale. Studi in onore di Sergio Zaninelli, a cura di A. CARERA, M. TACCOLInI, R. CANETTA, Milano, 1999; Atti di intelligenza e sviluppo economico: saggi per il bicentenario della nascita di Carlo Cattaneo, a cura di L. CAFAGNA e N. CREPAX, Bologna, 2001; E. Brambilla, Intervento su G. Alessi, La comparsa di una polizia "moderna", in La polizia in Italia nell'età moderna a cura di L. ANTONIELLI, Soveria Mannelli, 2002, p. 47 e nota 22 (ivi anche le relazioni di A. ConTINI, Quali le funzioni di polizia? pp. 65-80, p. 78 s., e di L. ANTONIELLI, Gli uomini della polizia e l'arruolamento, pp. 117-136, p. 132; Vincenzo Cuoco nella cultura di due secoli. Atti del convegno internazionale, Campobasso, 20-22 gennaio 2000, a cura di L. BISCARDI e A. De Francesco, Roma, 2002; M.G. Vitali-Volant, Cesare Beccaria (1738-1794) Cours et discours d'économie politique, Paris 2006; A. QuAdrIO CuRzIO, Economisti 
ed economia. Per un'Italia europea: paradigmi tra il XVIII e il XX secolo, Bologna, 2007; L. AnTONIELLI, Les réformes de la police en Lombardie au XVIII siècle, in Lombardie et Pays-Bas autrichiens. Regards croisés sur les Habsbourg et leurs réformes au XVIII ${ }^{e}$ siècle, in XVIII Études sur le $18^{e}$ siècle, 36 (2008), pp. 159-181; C. CAPRA, Risorgimento lombardo, in Archivio Storico Lombardo, CXXXVII (2011), pp. 11-23; M. PISANI, Cesare Beccaria e il principio di umanità, in Rivista italiana di diritto e procedura penale, 54 fasc. 2 (2011), pp. 407-413. 